\title{
Optical biosensors - Illuminating the path to personalized drug dosing
}

\author{
Jun Jie Ong ${ }^{\text {a }}$, Thomas D. Pollard ${ }^{\text {a }}$, Alvaro Goyanes ${ }^{\text {a,b }}$, Simon Gaisford ${ }^{\text {a }}$, \\ Mohammed Elbadawi ${ }^{\text {a }}$, Abdul W. Basit ${ }^{\text {a,* }}$ \\ a Department of Pharmaceutics, UCL School of Pharmacy, University College London, 29-39 Brunswick Square, London, WC1N 1AX, United Kingdom \\ b Departamento de Farmacología, Farmacia y Tecnología Farmacéutica, I+D Farma Group (GI-1645), Universidade de Santiago de Compostela, 15782, Spain
}

\section{A R T I C L E I N F O}

\section{Keywords:}

Precision medicine

Therapeutic drug monitoring

Digital healthcare

Personalized biopharmaceuticals and drug

products

Industry 4.0

Translational pharmaceutical sciences

\begin{abstract}
A B S T R A C T
Optical biosensors are low-cost, sensitive and portable devices that are poised to revolutionize the medical industry. Healthcare monitoring has already been transformed by such devices, with notable recent applications including heart rate monitoring in smartwatches and COVID-19 lateral flow diagnostic test kits. The commercial success and impact of existing optical sensors has galvanized research in expanding its application in numerous disciplines. Drug detection and monitoring seeks to benefit from the fast-approaching wave of optical biosensors, with diverse applications ranging from illicit drug testing, clinical trials, monitoring in advanced drug delivery systems and personalized drug dosing. The latter has the potential to significantly improve patients' lives by minimizing toxicity and maximizing efficacy. To achieve this, the patient's serum drug levels must be frequently measured. Yet, the current method of obtaining such information, namely therapeutic drug monitoring (TDM), is not routinely practiced as it is invasive, expensive, time-consuming and skilled labor-intensive. Certainly, optical sensors possess the capabilities to challenge this convention. This review explores the current state of optical biosensors in personalized dosing with special emphasis on TDM, and provides an appraisal on recent strategies. The strengths and challenges of optical biosensors are critically evaluated, before concluding with perspectives on the future direction of these sensors.
\end{abstract}

\section{Introduction}

Personalized medicine is the contemporary approach towards disease management and treatment which forsakes conventional one-sizefits-all models for bespoke medicines tailored to the individual. Its rise to prominence was brought forth by the knowledge generated through the Human Genome Project (Carrasco-Ramiro et al., 2017). The growing molecular understanding of diseases has not only improved diagnostic strategies but has also informed us of the subtle variations in the way different people respond to medicines. This should hardly come as a surprise; sex differences in digoxin toxicity, for instance, were reported as early as 1964 (Rodensky and Wasserman, 1964). Today, we are better equipped to understand the molecular mechanisms underlying interand intra-patient variations, such as sex (Farkouh et al., 2020; Madla et al., 2021), metabolic enzymes polymorphism (Ahmed et al., 2016), gut microbiota (McCoubrey et al., 2021; Zimmermann et al., 2019), and the time of administration (Zaki et al., 2019). Progress towards tailor-made medicines is faster than ever due in part to political interest and pressure, with the 2015 Precision Medicine Initiative launched by the Obama administration (Fox, 2015) one key example of this.

Personalized medicine encompasses numerous concepts, including patient-centric dose design and personalized dosing. Advances in 3D printing technologies have enabled the fabrication of precise doses for any given individual (Capel et al., 2018; Elbadawi et al., 2020; Eleftheriadis et al., 2021; Goyanes et al., 2019; Melocchi et al., 2021; Seoane-Viaño et al. 2021a, 2021b). To determine the optimal dose to be administered, a means of determining the patient's drug serum concentration is necessary. This can be achieved through therapeutic drug monitoring (TDM). TDM is the clinical practice of measuring the extent to which a patient has been exposed to a therapeutic agent, known as the exposure parameter, to adjust subsequent doses and optimize dosing regimens (Ates et al., 2020). Different exposure parameters may be used for different types of drugs, such as the trough concentration (for antiepileptics or antivirals), peak concentration (for centration-depen dent antibiotics), and the area under time-concentration curve (AUC) (for immunosuppressants) (Buclin et al., 2020). These exposure parameters are interpreted using dosing adaptation algorithms derived from pharmacokinetic-pharmacodynamic (PK-PD) models, which are in turn

\footnotetext{
* Corresponding author.

E-mail address: a.basit@ucl.ac.uk (A.W. Basit).
} 
built from population studies on the inter- and intra-patient pharmacokinetic variability for the specific drug (Jelliffe et al., 2020; Stillhart et al., 2020; Vinarov et al., 2021). As these existing PK-PD models are representative of an average patient rather than an individual, dose adjustments through present-day TDM practices are not truly personalized (Ates et al., 2020). A tailored dosage regimen will require continuous or frequent monitoring of the aforementioned exposure parameters to both construct an individualized PK-PD model for each patient and accurately evaluate the patient's drug exposure. However, at present, TDM practices require the invasive sampling of blood, which can be an unpleasant experience when done routinely. Additionally, blood samples are analyzed using expensive chromatographic or immunoassay-based machines, at times in a centralized laboratory. These result in long turnaround times, high instrumentation costs and intensive skilled labor requirements (Ates et al., 2020). Prompt and routine TDM is consequently unfeasible, limiting its present-day use to narrow therapeutic index (NTI) drugs (i.e. drugs with a small gap between their therapeutic and toxic concentrations).

Indeed, TDM possesses immense potential that largely remains untapped (Garzón et al., 2019). Knowledge of drug serum concentrations through TDM allow clinicians and pharmacist to adjust the next scheduled dose, ensuring that drug concentrations are kept within the therapeutic window. As such, adverse and occasionally fatal side effects are avoided, and the Time in Therapeutic Range (TTR) can be maximized to improve patient outcomes. In this way, TDM may also help to mitigate intra and/or inter-patient variability. If its application is extended beyond NTI drugs, the amplification of these benefits will likely see reduced drug wastage and reduced hospitalization due to avoidable drug overdoses. Routine TDM practices for antibiotics may also help to tackle the pressing issue of antimicrobial resistance by obviating sub-therapeutic doses.

Given its benefits, it is impetuous to disregard the notion of enabling timely, routine and affordable TDM practices. Optical biosensors could hold the key to unlocking the full potential of TDM (Garzón et al., 2019). A biosensor is a device that employs specific biological elements (such as enzymes, antibodies, aptamers) and/or biochemical reactions to detect specific chemical analytes through the quantification of optical, thermal, electrical or piezoelectric signals (Elbadawi et al., 2021; Nagel et al., 1992). Optical biosensors use the interaction of light (electromagnetic radiation/waves) with matter to measure the concentration of a species. The interaction can be measured through adsorption, scattering (absorbing and re-emitting the light), refractive index changes and fluorescence (Chen and Wang, 2020). Optical sensors have already been established as a staple of the healthcare industry, ranging from traditional sensors such as pulse oximeters to modern photoplethysmograms for heart rate monitoring in wearable devices such as the Apple Watch and Fitbit. Today, optical biosensors continue to evolve in many applications, including food safety (Scognamiglio et al., 2014), pathogen detection (Yoo and Lee, 2016), cancer diagnosis (Balaji and Zhang, 2017), environmental monitoring (Halilović et al., 2019; Liu et al., 2019), DNA sensing (Lan et al., 2019), and blood glucose monitoring (Mendosa, 2000). Indeed, optical biosensors are steadily permeating nearly every aspect of our lives. Consequently, its implementation into practices with stricter regulatory requirement, such as TDM, becomes increasingly likely.

In the following review, we provide an overview of the key advances in optical biosensors for drug detection and quantification, and discuss their potential to enable routine and cost-effective TDM. In particular, we discuss the overarching principles that underpin various optical biosensors and critically review recent examples with potential for clinical translation. We examine the pros and cons of optical biosensors, including the technical and regulatory challenges that must be addressed to permit their translation into clinical practice.

\section{Visualizing the molecular world: the overarching principles}

Optical biosensors share a common set of working components. Foremost, to capture the analyte of interest, a bio-recognition element is necessary. This can be organic such as enzymes, antibodies, aptamers, cells, or tissues (Chen and Wang, 2020), or inorganic such as molecularly imprinted polymers (MIPs) (Uzun and Turner, 2016). Interactions between the bio-recognition element and the analyte lead to a signal through interactions with light, which can originate from a light source such as a laser or LED. These interactions include fluorescence, absorption, refraction index or light scattering. The signal produced is subsequently correlated to the concentration of the measured analyte. A waveguide, such as a fiber optic (Bosch et al., 2007), is included to guide the light or to produce evanescent waves (Duval and Lechuga, 2015). A detection array, such as a photodiode, light-dependent resistor (LDR), phototransistor or charged coupled device (CCD), captures and quantifies the signal. Finally, a signal processing unit converts the measured signal into readable information for the users, which in this case is the concentration of the analyte.

Often, plasmonic nanomaterials may be used due to their unique optical properties (Jouyban and Rahimpour, 2020); these unique optical properties are a result of their shape and size. Further, labels, which are molecules which interact with the light to producing a measurable signal (JoVE Science Education Database, 2020), can be used. Optical biosensors can be broadly classified as label-free (detection signal is generated from the interaction of the analyte with the transducer) or label-based (uses a label, and the optical signal is generated by a colorimetric or luminescent method) (Damborský et al., 2016). To improve the measured signal, other optical elements, such as lenses for focusing the beam or filters for removing noise, may also be included. Further detailed explanation on the mechanisms that underlie optical biosensors is outside the scope of this review, but can be found in a recently published review (Chen and Wang, 2020).

Optical biosensors are the most commonly reported class of biosensors and a wide range of techniques have been explored (Damborský et al., 2016). Some of the prominent technologies that have dominated the field of optical biosensors include (1) spectrophotometry, (2) fluorimetry, (3) Surface Enhanced Raman Spectroscopy (SERS), (4) chemiluminescence, and (5) Surface Plasmon Resonance (SPR) (Table 1; Fig. 1). These will be discussed further in the following section.

\section{Surveying the current state of optical biosensors}

Optical biosensors have had a long-standing presence in the healthcare sector. Lateral flow immunoassays or immunochromatographic test strips are perhaps the earliest forms of optical biosensors. These include home pregnancy test kits and most recently, SARS-CoV-2 diagnostic kits (Udugama et al., 2020). Here, biorecognition molecules are conjugated with colored compounds (e.g. gold nanoparticles) and migrate across the test strip via capillary action (Koczula and Gallotta, 2016). Antibodies specific for the analyte are immobilized in a horizontal line onto the test zone. Binding of the analyte-biorecognition element complex to the immobilized antibodies produces a colored line that becomes more visible with increasing analyte concentration. Excess biorecognition molecules migrate further until it reaches the control zone, wherein antibodies specific for the biorecognition molecules are immobilized. Consequently, a clear line formed in the control and test zone is indicative of a positive test, while a single line in the control zone is indicative of a negative test. Another optical sensor that is actively used in hospitals is the pulse oximeter, which enables the monitoring of patients' oxygen saturation levels (Jubran, 2015).

Indeed, the successful and routine use of traditional optical biosensors has galvanized research into next-generation sensors for diverse healthcare applications. Most research today focuses on developing optical lab-on-chip sensors that share similar characteristics with their aforementioned predecessors: portable, affordable, reliable and fast. For 
Table 1

A brief overview of the working principles of the five optical biosensing techniques discussed in this review.

\begin{tabular}{|c|c|c|}
\hline Biosensor technique & Working principle & Measured signal \\
\hline Spectrophotometry & $\begin{array}{l}\text { Measures the absorbance of } \\
\text { light in the visible, infra-red, } \\
\text { or UV spectrum, which is then } \\
\text { correlated to the } \\
\text { concentration of the analyte } \\
\text { by Beer-Lambert's law }\end{array}$ & $\begin{array}{l}\text { Absorbance intensity } \\
\text { (in visible, infra-red, } \\
\text { or UV spectrum) }\end{array}$ \\
\hline Fluorimetry & $\begin{array}{l}\text { A molecule known as a } \\
\text { fluorophore absorbs } \\
\text { electromagnetic radiation and } \\
\text { re-emits light at a different } \\
\text { wavelength. The intensity of } \\
\text { the re-emitted light and time } \\
\text { decay correlates to the } \\
\text { concentration of the molecule. }\end{array}$ & Fluorescence intensity \\
\hline $\begin{array}{l}\text { Surface Enhanced } \\
\text { Raman Scattering } \\
\text { (SERS) }\end{array}$ & $\begin{array}{l}\text { Raman scattering occurs when } \\
\text { light is scattered inelastically } \\
\text { when it interacts with and } \\
\text { excites a molecule. This } \\
\text { produces a spectrum unique to } \\
\text { a molecule, wherein the } \\
\text { intensity of the peaks is } \\
\text { directly proportional to the } \\
\text { concentration of the molecule. } \\
\text { As Raman scattering gives } \\
\text { weak signals, rough metal } \\
\text { surfaces are used to amplify } \\
\text { the signal, thereby giving } \\
\text { Surface Enhanced Raman } \\
\text { Spectroscopy }\end{array}$ & $\begin{array}{l}\text { Intensity of peaks in } \\
\text { Raman spectrum }\end{array}$ \\
\hline Chemiluminescence & $\begin{array}{l}\text { Measures the intensity of light } \\
\text { produced from a chemical } \\
\text { reaction. }\end{array}$ & Light intensity \\
\hline $\begin{array}{l}\text { Surface Plasmon } \\
\text { Resonance }\end{array}$ & $\begin{array}{l}\text { Surface plasmons, which are } \\
\text { oscillations of free electrons at } \\
\text { the interface of a metal and } \\
\text { dielectric, are generated when } \\
\text { polarized light strikes the } \\
\text { conductive surface. Binding of } \\
\text { an analyte to its corresponding } \\
\text { biorecognition element } \\
\text { immobilized on the metal } \\
\text { surface causes a change in the } \\
\text { refractive index of the } \\
\text { dielectric. This consequently } \\
\text { affects the propagation } \\
\text { constant of the surface } \\
\text { plasmons, which then results } \\
\text { in concentration-dependent } \\
\text { shift of resonance angle } \\
\text { (angular modulation) or } \\
\text { resonance wavelength } \\
\text { (wavelength modulation). }\end{array}$ & $\begin{array}{l}\text { Change in refractive } \\
\text { index }\end{array}$ \\
\hline
\end{tabular}

instance, Brian Gitta's Matibabu, a non-invasive low-cost malaria diagnosis device, draws inspiration from present-day pulse oximeters (BBC News, 2018). Like its progenitor, Matibabu clips onto a patient's finger and shines a red light through the skin. This light is used to detect changes in the red blood cells of infected patients, specifically the presence of hemozoin crystals excreted by malaria parasites. Test results are sent within 1-2 min through a mobile phone application, mitigating the long turnover time characteristic of conventional diagnosis procedures. This example also draws attention to a technology symbolic of the 21st century that might hold the key to achieving accessible optical biosensors: the smartphone. With incremental improvements in processing capabilities and camera quality in each yearly iteration, smartphones have been explored as potential replacements for traditional analytical machines (Guner et al., 2017; Hossain et al., 2015; Ruppert et al., 2019). This will be discussed further in a later section.

With regards to TDM, it is regrettable that commercial TDM optical biosensors have yet to enter clinical practice. Nonetheless, the clinical success of diagnosis and healthcare monitoring optical biosensors has encouraged the use of optical biosensors for TDM. Considerable research is being undertaken towards the development of such biosensors, with equal effort being devoted to each salient configuration. An updated list of reported optical biosensors for TDM is enumerated in Table 2, and each optical technique is discussed in more detail in individual subsections hereon.

\subsection{Spectrophotometry}

Spectrophotometry measures the absorption of light as it passes through a solution. By using the Beer-Lambert law, which states that the absorbance is directly proportional to the concentration of the analyte (Ball, 2006), the concentration of analyte in a solution can be quantified. Spectrophotometry can be sub-divided according to the wavelength of the measured light. These include visible spectrometry (also known as colorimetry), UV spectrometry, and infra-red spectrometry (Germer et al., 2014). Traditionally, colorimetry has been used for qualitative purposes, e.g. in lateral flow immunoassays as aforementioned. However, by measuring the intensity of visible light at a specific wavelength, quantitative applications are also possible. While drug molecules may not inherently absorb nor emit light in the visible spectrum, colorimetric approaches are made possible by conjugating signal transducers (e.g. gold or silver nanoparticles) to biorecognition elements.

In numerous studies, this strategy has been demonstrated through the use of laboratory-grade spectrophotometers in conjunction with biorecognition elements. Various research groups have modified the surface of noble metal, such as gold and silver, nanoparticles with small molecules that interact favourably to the target analyte. For instance, amidosulfonic acid-capped silver nanoparticles (ASA-AgNPs) were used for the visible spectrophotometric detection of lamotrigine, an antiepileptic drug (Jouyban et al., 2017b). The amine groups of amidosulfonic acid interact with lamotrigine through cooperative hydrogen bonding. These modified AgNPs are normally dispersed and give a yellow color, but in the presence of lamotrigine, the ASA-AgNPs aggregate and turn red (Fig. 2). Subsequently, further aggregation produces a violet color. The color change was monitored by measuring the ratio of absorbance at $450 \mathrm{~nm}-390 \mathrm{~nm}$ after $30 \mathrm{~min}$ of incubation of the ASA-AgNPs and sample using a UV-2550 spectrophotometer. This was applied to the measurement of lamotrigine in both spiked and dosed epileptic patient exhaled breath condensate. This showed no statistical difference between this method and HPLC measurements, with recoveries of 96.5-107.1\%. This method also showed a moderate degree of selectivity, as some co-administered antiepileptics, including, sodium valproate, phenobarbital and carbamazepine, were shown to not interfere with the assay. Nevertheless, the authors admitted that phenytoin was found to be an interferant, and therefore lamotrigine cannot be accurately quantified in the presence of phenytoin.

The above example demonstrates how small molecules, while affordable and robust, do not provide a sufficient degree of selectivity for practical clinical use. Consequently, biologically derive constructs, such as antibodies, enzymes, and aptamers, have been explored as highly selective and sensitive bioreceptors (Zahra et al., 2021). For example, gold nanoparticles (AuNPs) were employed in conjunction with digoxin aptamers for the quantification of digoxin (Sarreshtehdar Emrani et al., 2015). In the absence of digoxin, the negatively charged surface of AuNPs adsorbs the positively charged bases of aptamers via electrostatic interaction. This stabilizes the AuNPs, protecting them from salt-induced aggregating and causing the solution to appear wine-red in color. When digoxin is added, the aptamers conjugates to digoxin and detaches from the surface of AuNPs, which subsequently aggregates upon the addition of sodium chloride. This causes the color of the solution to turn blue. Here, the absorbance at $520 \mathrm{~nm}$ was measured using a Synergy $\mathrm{H} 4$ microplate reader. Using this approach, an LOD of $2.37 \mathrm{nM}(1.85 \mathrm{ng} / \mathrm{mL})$ in serum was achieved, which is lower than the toxicity levels of digoxin in blood $(2 \mathrm{ng} / \mathrm{mL})$. This aptasensor also 


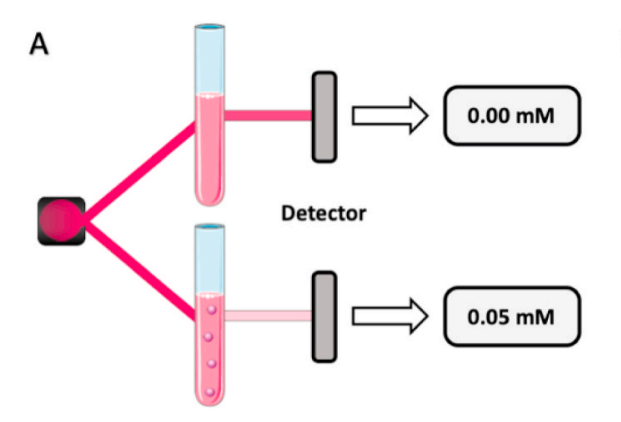

C

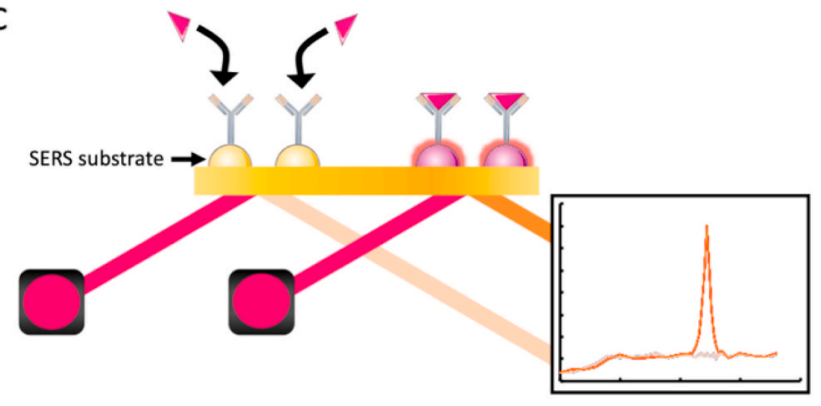

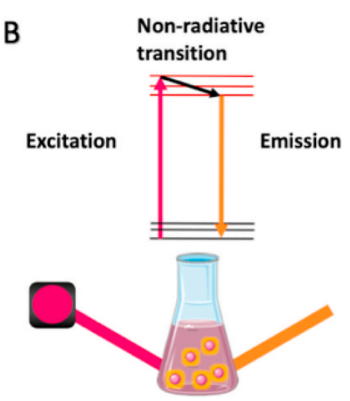
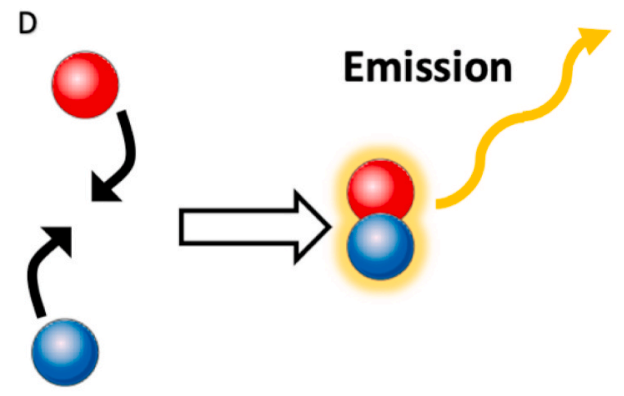

E

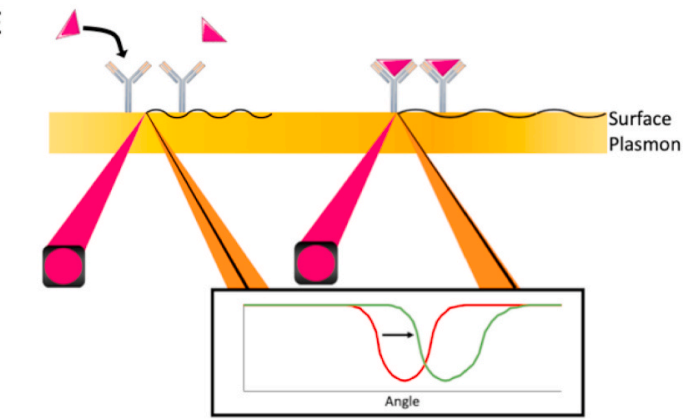

: Laser/light source $\quad$ _ Incident light $\quad$ _ Reflected/emitted light $\quad$ _ Analyte

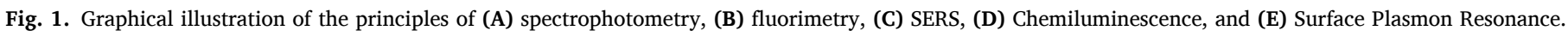

demonstrated notable selectivity, as no significant absorbance was observed in the presence of other drugs, including amoxicillin, ouabain, ibuprofen and acetylsalicylic.

While conventional spectrophotometers or plate readers are arguably more affordable than the equipment used in current TDM practices (i.e. LC-MS/MS, GC-MS/MS), these are still bulky and relatively expensive machinery that cannot be feasibly deployed at the point-ofcare (e.g. in pharmacies). Accordingly, researchers have explored the use of portable spectrophotometers and even smartphones as potential alternatives to enable affordable and decentralized TDM (Faham et al., 2019; Hossain et al., 2015; Kong et al., 2020). For instance, Faham et al. utilized a Samsung Galaxy E5 smartphone camera (8.0-megapixel camera) to monitor colorimetric changes in their nanopaper-based analytical device/curcumin-embedded in bacterial cellulose nanopaper (NAD/CEBC) bioplatform for the quantification of Fe(III) and deferoxamine. Here, curcumin is released from the bioplatform in the presence of Fe(III) due to the formation of Fe(III)-curcumin complex. This results in a decrease in color intensity. Deferoxamine, as an iron-chelating drug, competes with curcumin for binding to Fe(III), causing curcumin to bind back to the bioplatform and consequently increase the color intensity. To monitor the color changes, the smartphone was placed in a fabricated light control box comprising a paperboard dark chamber with white LED lamps. Images were captured with the smartphone camera and analyzed using Adobe Photoshop CS5 where the mean color intensity was measured. This smartphone-based approach achieved an LOD for deferoxamine of $8.2 \mathrm{nM}$, with an impressive RSD value of $\leq 3.6 \%$ (RSD value for spectrophotometer-based method was $\leq 2.5 \%$ and the LOD was $8.0 \mathrm{nM}$ ). While very slightly inferior in sensitivity and precision compared to the spectrophotometer, this study demonstrated the feasibility of using smartphone cameras for accurate drug quantification and TDM.

\subsection{Fluorimetry}

Fluorescence occurs when a molecule absorbs electromagnetic radiation and re-emits light at a different wavelength; the wavelength of the re-emitted light is characteristic of the molecule (Wang et al., 2017b), The intensity of the re-emitted light, measured using a fluorimeter or plate reader, is subsequently used to determine the concentration of the molecule. Compounds that exhibit fluorescence are known as fluorophores. Like visible spectroscopy, most drug molecules are not fluorophores and cannot be inherently measured through fluorimetry. Instead, a fluorophore (e.g. safranin) is often conjugated to the biorecognition element. Other systems may pair the fluorophore with a quenching agent, which is a substance that reduces the fluorescence intensity of the fluorophore. In these, the distance between the fluorophore and the quenching agent is altered as a result of the biorecognition event. This can occur in several ways, such as a conformational change in the biorecognition element due to analyte binding, or displacement of the fluorophore/quenching agent by the analyte. The concentration of the analyte is directly or indirectly proportional to the fluorescence intensity depending on the system's native state, i.e. the former if the biorecognition event causes the distance between the fluorophore and quenching agent to increase. Occasionally, the analyte of interest may itself act as a quenching agent.

Terbium (III) ion $\left(\mathrm{Tb}^{3+}\right)$ has been shown to give strong fluorescence when forming a complex with different species, which is further enhanced in the presence of silver nanoparticles. This has been used as a technique for TDM of catecholamines (Alam et al., 2012), fluoroquinolones (Kamruzzaman et al., 2011), sertraline (Lotfi et al., 2017), and fluoxetine in diluted urine (Lotfi and Manzoori, 2016); where recoveries were in the ranges of 96.3-101, 97.75-101.5, 97.75-101.2 and 98.06-102.25\%, respectively. In the case of sertraline, 1 , 10-phenanthroline was used to form a complex with $\mathrm{Tb}^{3+}$ for improved luminescent intensity, which was in turn measured using a RF-5301-PC spectrofluorophotometer at $545 \mathrm{~nm}$. Similarly, silver nanoparticles were used to enhance the fluorescent intensity of the deferiprone-terbium ion complex in exhaled breath condensate (EBC) samples (Mohamadian et al., 2017). Here, fluorescent intensity at 295 and $545 \mathrm{~nm}$ was measured using a JASCO FP-750 spectrofluorometer. Even though strong linear correlation $\left(\mathrm{R}^{2}=0.999\right)$ was achieved for regression between deferiprone concentration and fluorescence intensity, correlation between the administered dose and deferiprone concentration in EBC could not be established due to other confounding variables influencing the pharmacokinetic of deferiprone (e.g. time interval between sampling, gender). Furthermore, while the sensing strategy proved to 
Table 2

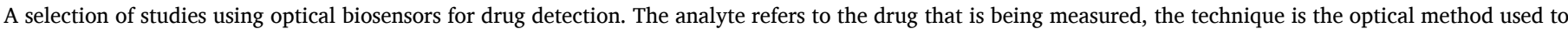

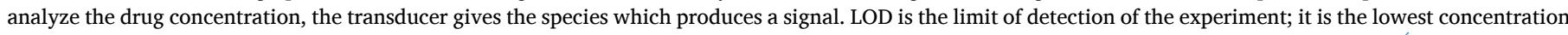

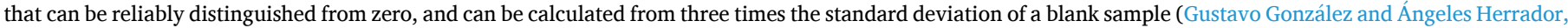

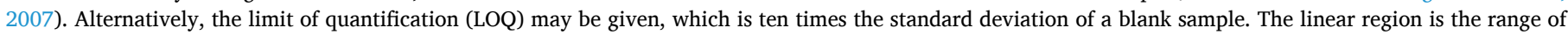

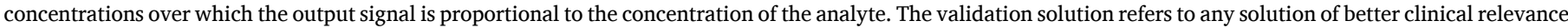
in which the drug was dissolved in.

\begin{tabular}{|c|c|c|c|c|c|c|c|}
\hline Analyte & Technique & Transducer & $\begin{array}{l}\text { Biorecognition } \\
\text { Element }\end{array}$ & LOD & $\begin{array}{l}\text { Detection } \\
\text { Range }\end{array}$ & $\begin{array}{l}\text { Validation } \\
\text { solution }\end{array}$ & Ref. \\
\hline \multicolumn{8}{|l|}{ Spectrophotometry } \\
\hline Lamotrigine & $\begin{array}{l}\text { UV-Vis } \\
\text { spectrometry }\end{array}$ & $\begin{array}{l}\text { Amidosulfonic acid- } \\
\text { capped AgNPs }\end{array}$ & Amidosulfonic acid & $5 \mathrm{ng} / \mathrm{ml}$ & $\begin{array}{l}0.02-0.4 \mu \mathrm{g} / \\
\mathrm{ml}(450: 390 \\
\mathrm{nm})\end{array}$ & $\begin{array}{l}\text { Exhaled breath } \\
\text { condensate }\end{array}$ & Jouyban et al. (2017b) \\
\hline $\begin{array}{l}\text { 6-aminopenicillanic } \\
\text { acid }\end{array}$ & $\begin{array}{l}\text { UV-Vis } \\
\text { spectrometry }\end{array}$ & $\begin{array}{l}\text { AgNPs, with thiopyridine } \\
\text { stabilizer }\end{array}$ & Thiopyridine & $1 \mu \mathrm{M}$ & $1-50 \mu \mathrm{M}$ & Plasma & Anwar et al. (2019) \\
\hline Nitrofurazone & $\begin{array}{l}\text { UV-Vis } \\
\text { spectrometry }\end{array}$ & Olive-oil based AgNPs & None & $1.88 \mu \mathrm{M}$ & $1-500 \mu \mathrm{M}$ & Plasma & Ahmed et al. (2018) \\
\hline Deferiprone & $\begin{array}{l}\text { UV-Vis } \\
\text { Spectrometry }\end{array}$ & $\begin{array}{l}\text { Pyrophosphate } \\
\text { functionalized AgNPs, } \\
\text { plus Fe(III) }\end{array}$ & $\begin{array}{l}\text { Fe(III) and } \\
\text { Pyrophospate }\end{array}$ & $280 \mathrm{nM}$ & $6-100 \mu \mathrm{M}$ & $\begin{array}{l}\text { Pre-treated and } \\
\text { diluted plasma }\end{array}$ & Chavada et al. (2017) \\
\hline Propafenone & $\begin{array}{l}\text { UV-Vis } \\
\text { Spectrometry }\end{array}$ & Citrate-capped AgNPs & Citrate ligand & $2.4 \mu \mathrm{M}$ & $10-35 \mu \mathrm{M}$ & $\begin{array}{l}\text { Pre-treated and } \\
\text { diluted urine }\end{array}$ & Qu et al. (2012) \\
\hline Etimicin & $\begin{array}{l}\text { UV-Vis } \\
\text { spectrometry }\end{array}$ & Citrate-capped AgNPs & Citrate ligand & $359 \mathrm{nM}$ & $375-575 \mathrm{nM}$ & Diluted urine & Li et al. (2014) \\
\hline Metformin & $\begin{array}{l}\text { UV-Vis } \\
\text { Spectrometry }\end{array}$ & $\begin{array}{l}\text { Cucurbit [6]uril - } \\
\text { modified AgNPs }\end{array}$ & $\begin{array}{l}\text { Cucurbit [6]uril } \\
\text { ligand }\end{array}$ & $1 \mu \mathrm{M}$ & $\begin{array}{l}3-150 \mu \mathrm{M}, \\
150-750 \mu \mathrm{M}\end{array}$ & $\begin{array}{l}\text { Pre-treated and } \\
\text { diluted urine }\end{array}$ & Song et al. (2019) \\
\hline Lomefloxacin & $\begin{array}{l}\text { UV-Vis } \\
\text { Spectrometry }\end{array}$ & Cystine - modified AgNPs & Cystine ligand & $100 \mathrm{nM}$ & $\begin{array}{l}200 \mathrm{nM}-5 \\
\mu \mathrm{M}\end{array}$ & Diluted urine & Gao et al. (2018b) \\
\hline R-Citalopram & $\begin{array}{l}\text { UV-Vis } \\
\text { Spectrometry }\end{array}$ & AgNPs & None & $3 \mathrm{nM}$ & $\begin{array}{l}7.9-270 \mathrm{nM}, \\
6.7-170 \mu \mathrm{M}\end{array}$ & None & $\begin{array}{l}\text { Tashkhourian and } \\
\text { Afsharinejad (2017) }\end{array}$ \\
\hline Chloramphenicol & Colorimetry & $\begin{array}{l}\text { AuNP - binding DNA } \\
\text { (ABD) }\end{array}$ & $\begin{array}{l}\text { Biotin-modified } \\
\text { Aptamer }\end{array}$ & $451 \mathrm{pM}$ & $\begin{array}{l}\text { Up to } \sim 120 \\
\mathrm{nM}\end{array}$ & Milk, serum & Abnous et al. (2016) \\
\hline Tetracycline & Colorimetry & $\begin{array}{l}\text { Modified tetracycline, } \\
\text { Signal Transduction } \\
\text { Probe and AuNP }\end{array}$ & $\begin{array}{l}\text { Modified tetracycline } \\
\text { aptamer }\end{array}$ & $266 \mathrm{pM}$ & $0.3-10 \mathrm{nM}$ & $\begin{array}{l}\text { Pre-treated } \\
\text { serum and milk }\end{array}$ & Ramezani et al. (2015) \\
\hline Digoxin & Colorimetry & $\begin{array}{l}\text { Aptamer-functionalized } \\
\text { AuNPs }\end{array}$ & Aptamer & $571 \mathrm{pM}$ & Up to $30 \mathrm{nM}$ & $\begin{array}{l}\text { Diluted Rat } \\
\text { Serum }\end{array}$ & $\begin{array}{l}\text { Sarreshtehdar Emrani } \\
\text { et al. (2015) }\end{array}$ \\
\hline Deferoxamine & $\begin{array}{l}\text { UV-Vis } \\
\text { Spectrometry }\end{array}$ & $\begin{array}{l}\text { Curcumin-embedded } \\
\text { bacterial cellulose }+\mathrm{Fe} \\
\text { (III) }\end{array}$ & Curcumin & $8.2 \mathrm{nM}$ & $0.01-100 \mu \mathrm{M}$ & Saliva & Faham et al. (2019) \\
\hline Carbamazepine & $\begin{array}{l}\text { UV-Vis } \\
\text { Spectrometry }\end{array}$ & $\begin{array}{l}\text { Horseradish-peroxidase } \\
\& 3,3^{\prime}, 5,5^{\prime} \text { - } \\
\text { tetramethylbenzidine } \\
\text { oxidation }\end{array}$ & Anti-CBZ antibodies & $1.0 \mu \mathrm{M}$ & $1.0-50 \mu \mathrm{M}$ & $\begin{array}{l}\text { Spiked human } \\
\text { serum }\end{array}$ & Ramos et al. (2019) \\
\hline Gentamicin & $\begin{array}{l}\text { UV-Vis } \\
\text { Spectroscopy }\end{array}$ & $\begin{array}{l}\text { Antibody-functionalized } \\
\text { Au Nanorods }\end{array}$ & $\begin{array}{l}\text { Anti-Gentamicin } \\
\text { antibodies }\end{array}$ & $0.05 \mathrm{ng} / \mathrm{ml}$ & $0.1-20 \mathrm{ng} / \mathrm{ml}$ & None & Zhu et al. (2011) \\
\hline Tetracycline & Colorimetry & $\begin{array}{l}\text { Horseradish Peroxidase } \\
\text { and } 3,3^{\prime}, 5,5^{\prime} \text { - }\end{array}$ & Antibodies & $0.5 \mathrm{ng} / \mathrm{ml}$ & $\begin{array}{l}0.5 \mathrm{ng} / \mathrm{ml}- \\
10 \mu \mathrm{g} / \mathrm{ml}\end{array}$ & $\begin{array}{l}\text { Milk and Fish } \\
\text { Samples }\end{array}$ & Wang et al. (2021) \\
\hline Chloramphenicol & & $\begin{array}{l}\text { Tetramethylbenzidine } \\
\text { oxidation }\end{array}$ & & $0.05 \mathrm{ng} / \mathrm{ml}$ & $\begin{array}{l}0.05 \mathrm{ng} / \mathrm{ml}- \\
1 \mu \mathrm{g} / \mathrm{ml}\end{array}$ & & \\
\hline Kanamycin & Colorimetry & AuNPs & Hairpin DNA Probe & $0.68 \mu \mathrm{M}$ & $1-40 \mu \mathrm{M}$ & $\begin{array}{l}\text { Spiked Milk } \\
\text { Samples }\end{array}$ & Xu et al. (2019) \\
\hline Amoxicillin & Colorimetry & Liquid Crystal layer & Aptamer & $3.5 \mathrm{nM}$ & $10-800 \mathrm{nM}$ & $\begin{array}{l}\text { Spiked River } \\
\text { and Tap Water }\end{array}$ & $\begin{array}{l}\text { Nguyen and Jang } \\
\text { (2021) }\end{array}$ \\
\hline Oxytetracycline & Colorimetry & APTES and $\mathrm{Cu}(\mathrm{II})$ & None & Not given & $\begin{array}{l}500 \mathrm{nM}-5 \\
\mathrm{mM}\end{array}$ & $\begin{array}{l}\text { Environmental } \\
\text { Water }\end{array}$ & $\begin{array}{l}\text { Gomes and Sales } \\
(2015)\end{array}$ \\
\hline Gentamicin & Colorimetry & AuNPs & Cysteamine & $12.45 \mathrm{nM}$ & Up to $200 \mathrm{nM}$ & $\begin{array}{l}\text { Pretreated Milk } \\
\text { Samples }\end{array}$ & $\begin{array}{l}\text { Gukowsky et al. } \\
\text { (2018) }\end{array}$ \\
\hline Amascrine & $\begin{array}{l}\text { UV-Vis } \\
\text { Spectroscopy }\end{array}$ & None & dsDNA & Not given & $5-25 \mu \mathrm{M}$ & None & $\begin{array}{l}\text { Akbari Javar et al. } \\
(2020)\end{array}$ \\
\hline \multicolumn{8}{|l|}{ Fluorimetry } \\
\hline SN-38 & Fluorimetry & None & None & $1.5 \mathrm{ng} / \mathrm{ml}$ & $\begin{array}{l}10-500 \mathrm{ng} / \\
\mathrm{ml}\end{array}$ & $\begin{array}{l}\text { Pre-treated } \\
\text { plasma }\end{array}$ & Tartaggia et al. (2018) \\
\hline Lamotrigine & FRET & $\begin{array}{l}\text { 4-aminothiophenol- } \\
\text { stabilized AuQDs }\end{array}$ & Aminosulfonic Acid & $4 \mathrm{ng} / \mathrm{ml}$ & $\begin{array}{l}20-500 \mathrm{ng} / \\
\mathrm{ml}\end{array}$ & $\begin{array}{l}\text { Pre-treated and } \\
\text { diluted patient } \\
\text { plasma }\end{array}$ & Jouyban et al. (2017a) \\
\hline Sulfadiazine & Fluorimetry & AgNPs on graphene QD & None & $10 \mathrm{nM}$ & $\begin{array}{l}40 \mathrm{nM}-22 \\
\mu \mathrm{M}\end{array}$ & $\begin{array}{l}\text { Pre-treated and } \\
\text { diluted serum, } \\
\text { pre-treated } \\
\text { urine }\end{array}$ & $\begin{array}{l}\text { Afsharipour et al. } \\
\text { (2019) }\end{array}$ \\
\hline 6 - Thioguanine & FRET & Harmine and AgNPs & Harmine & $9.7 \mathrm{nM}$ & $15-750 \mathrm{nM}$ & $\begin{array}{l}\text { Pretreated and } \\
\text { diluted plasma }\end{array}$ & $\begin{array}{l}\text { Amjadi and } \\
\text { Farzampour (2014) }\end{array}$ \\
\hline Levofloxacin & Fluorimetry & Te(III) and AgNPs & None & $7.19 \mathrm{aM}$ & $\begin{array}{l}41.6 \mathrm{aM}- \\
3.59 \mathrm{fM}\end{array}$ & $\begin{array}{l}\text { Diluted urine, } \\
\text { pre-treated and }\end{array}$ & $\begin{array}{l}\text { Kamruzzaman et al. } \\
\text { (2011) }\end{array}$ \\
\hline Moxifloxacin & & & & $8.47 \mathrm{aM}$ & $\begin{array}{l}49.8 \mathrm{aM}- \\
2.49 \mathrm{fM}\end{array}$ & diluted serum & \\
\hline
\end{tabular}


Table 2 (continued)

\begin{tabular}{|c|c|c|c|c|c|c|c|}
\hline Analyte & Technique & Transducer & $\begin{array}{l}\text { Biorecognition } \\
\text { Element }\end{array}$ & LOD & $\begin{array}{l}\text { Detection } \\
\text { Range }\end{array}$ & $\begin{array}{l}\text { Validation } \\
\text { solution }\end{array}$ & Ref. \\
\hline Epinephrine & \multirow[t]{3}{*}{ Fluorimetry } & \multirow[t]{3}{*}{ Te(III) and AgNPs } & \multirow[t]{3}{*}{ None } & $0.25 \mathrm{nM}$ & $2.5-110 \mathrm{nM}$ & \multirow{3}{*}{$\begin{array}{l}\text { Pre-treated and } \\
\text { diluted serum, } \\
\text { diluted urine }\end{array}$} & \multirow[t]{3}{*}{ Alam et al. (2012) } \\
\hline Norepinephrine & & & & $0.64 \mathrm{nM}$ & $2.8-240 \mathrm{nM}$ & & \\
\hline Dopamine & & & & $0.42 \mathrm{nM}$ & $2.4-140 \mathrm{nM}$ & & \\
\hline Fluoxetine & Fluorimetry & Te(III) and AgNPs & None & $0.83 \mathrm{ng} / \mathrm{ml}$ & $\begin{array}{l}0.008-19 \mu \mathrm{g} / \\
\mathrm{ml}\end{array}$ & $\begin{array}{l}\text { Pre-treated and } \\
\text { diluted patient } \\
\text { blood, diluted } \\
\text { patient urine }\end{array}$ & $\begin{array}{l}\text { Lotfi and Manzoori } \\
\text { (2016) }\end{array}$ \\
\hline Sertraline & Fluorimetry & $\begin{array}{l}\text { 1,10- phenanthroline-Te } \\
\text { (III) and AgNPs }\end{array}$ & 1,10-phenanthroline & $290 \mathrm{pg} / \mathrm{ml}$ & $\begin{array}{l}1 \mathrm{ng} / \mathrm{ml} \mathrm{-} 3 \\
\mu \mathrm{g} / \mathrm{ml}\end{array}$ & $\begin{array}{l}\text { Pre-treated and } \\
\text { diluted patient } \\
\text { plasma, pre- } \\
\text { treated patient } \\
\text { urine }\end{array}$ & Lotfi et al. (2017) \\
\hline Deferiprone & Fluorimetry & Te (III) and AgNPs & None & $\begin{array}{l}0.06 \mu \mathrm{g} / \mathrm{ml} \\
(\mathrm{LOQ})\end{array}$ & $\begin{array}{l}0.06-1.5 \mu \mathrm{g} / \\
\mathrm{ml}\end{array}$ & $\begin{array}{l}\text { Exhaled breath } \\
\text { condensate }\end{array}$ & $\begin{array}{l}\text { Mohamadian et al. } \\
\text { (2017) }\end{array}$ \\
\hline Pefloxacin Mesylate & Fluorimetry & Te (III) and AgNPs & None & $0.25 \mathrm{nM}$ & $\begin{array}{l}0.8 \mathrm{nM}-1.00 \\
\mu \mathrm{M} \\
1.00-8.00 \\
\mu \mathrm{M}\end{array}$ & $\begin{array}{l}\text { Pre-treated and } \\
\text { diluted serum }\end{array}$ & Li and Song (2014) \\
\hline Teicoplanin & Fluorimetry & AgNPs & None & $0.16 \mathrm{ng} / \mathrm{ml}$ & $0.6-30 \mathrm{ng} / \mathrm{ml}$ & $\begin{array}{l}\text { Pre-treated and } \\
\text { diluted patient } \\
\text { plasma }\end{array}$ & Ali et al. (2019) \\
\hline Amikacin & Fluorimetry & Safranin & Safranin & $1.2 \mathrm{pg} / \mathrm{ml}$ & $4-60 \mathrm{pg} / \mathrm{ml}$ & $\begin{array}{l}\text { Pre-treated, } \\
\text { diluted serum } \\
\text { and patient } \\
\text { serum }\end{array}$ & Omar et al. (2013) \\
\hline Tobramycin & & & & $1.5 \mathrm{pg} / \mathrm{ml}$ & $5-50 \mathrm{pg} / \mathrm{ml}$ & None & \\
\hline Neomycin & & & & $1.2 \mathrm{pg} / \mathrm{ml}$ & $4-50 \mathrm{pg} / \mathrm{ml}$ & None & \\
\hline Gentamicin & & & & $1.2 \mathrm{pg} / \mathrm{ml}$ & $4-50 \mathrm{pg} / \mathrm{ml}$ & $\begin{array}{l}\text { Pre-treated, } \\
\text { diluted serum } \\
\text { and patient } \\
\text { serum }\end{array}$ & \\
\hline Kanamycin & & & & $1.2 \mathrm{pg} / \mathrm{ml}$ & $4-50 \mathrm{pg} / \mathrm{ml}$ & None & \\
\hline Streptomycin & & & & $1.2 \mathrm{pg} / \mathrm{ml}$ & $4-40 \mathrm{pg} / \mathrm{ml}$ & None & \\
\hline Paclitaxel & Fluorimetry & $\begin{array}{l}\text { Rhodamine-labelled } \\
\text { Paclitaxel }\end{array}$ & $\begin{array}{l}\text { Anti-Paclitaxel } \\
\text { antibody }\end{array}$ & $1.7 \mathrm{ng} / \mathrm{ml}$ & $\begin{array}{l}20-100 \mathrm{ng} / \\
\mathrm{ml}\end{array}$ & Diluted plasma & $\begin{array}{l}\text { Sheikh and } \\
\text { Mulchandani (2001) }\end{array}$ \\
\hline Methotrexate & Fluorimetry & N,S- codoped C Nanodots & None & $0.33 \mathrm{nM}$ & $1 \mathrm{nM}-50 \mu \mathrm{M}$ & $\begin{array}{l}\text { Pre-treated, } \\
\text { diluted serum }\end{array}$ & Wang et al. (2015) \\
\hline Tetracycline & Fluorimetry & $\begin{array}{l}\text { Modified tetracycline } \\
\text { aptamer and Signal } \\
\text { Transduction Probe }\end{array}$ & $\begin{array}{l}\text { Modified tetracycline } \\
\text { aptamer }\end{array}$ & $2.09 \mathrm{nM}$ & Up to $300 \mathrm{nM}$ & $\begin{array}{l}\text { Pre-treated rat } \\
\text { serum }\end{array}$ & Jalalian et al. (2015) \\
\hline Digoxin & Fluorimetry & $\begin{array}{l}\text { ATTO } 647 \mathrm{~N} \text {-labelled } \\
\text { aptamer functionalized } \\
\text { AuNPs. }\end{array}$ & $\begin{array}{l}\text { ATTO 64N-labelled } \\
\text { aptamer }\end{array}$ & $392 \mathrm{pM}$ & Up to $30 \mathrm{nM}$ & $\begin{array}{l}\text { Diluted rat } \\
\text { serum }\end{array}$ & $\begin{array}{l}\text { Sarreshtehdar Emrani } \\
\text { et al. (2015) }\end{array}$ \\
\hline Teicoplanin & Fluorimetry & Ninhydrin & $\begin{array}{l}\text { Salting Out Liquid- } \\
\text { liquid extraction }\end{array}$ & $\begin{array}{l}10.84 \mathrm{ng} / \\
\mathrm{mL}\end{array}$ & $\begin{array}{l}60-600 \mathrm{ng} / \\
\mathrm{mL}\end{array}$ & Human plasma & Ali et al. (2020) \\
\hline Rifampicin & Fluorimetry & $\begin{array}{l}\text { Glutathione-stabilized } \\
\text { copper nanocluster }\end{array}$ & Glutathione & $16 \mathrm{pM}$ & $\begin{array}{l}50-10,000 \\
\mathrm{pM}\end{array}$ & $\begin{array}{l}\text { Huma serum } \\
\text { samples }\end{array}$ & Wu et al. (2020) \\
\hline Cefixime & Fluorimetry & $\begin{array}{l}\mathrm{Cd}^{2+}-2,3- \\
\text { Pyridinecarboxylate/ } \\
\mathrm{Tb}^{3+} \text { Nanosheet }\end{array}$ & None & $26.7 \mathrm{nM}$ & $40-200 \mathrm{nM}$ & $\begin{array}{l}\text { Filtered River/ } \\
\text { Tap water }\end{array}$ & Qin et al. (2020) \\
\hline Sulfadiazine & Fluorimetry & SiNPs & None & $1.02 \mu \mathrm{M}$ & Up to $800 \mu \mathrm{M}$ & None & Bai et al. (2020) \\
\hline Norfloxacin & Fluorimetry & $\begin{array}{l}\text { Aggregate Induced } \\
\text { Emission Fluorescence } \\
\text { Nanospheres }\end{array}$ & Antibody & $0.08 \mathrm{ng} / \mathrm{ml}$ & $0.1-20 \mathrm{ng} / \mathrm{ml}$ & $\begin{array}{l}\text { Animal-derived } \\
\text { foods }\end{array}$ & Hu et al. (2020) \\
\hline Cisplatin & Fluorimetry & Thioflavin $\mathrm{T}$ & G quadruplex DNA & $10 \mathrm{nM}$ & $10-500 \mathrm{nM}$ & $\begin{array}{l}\text { Pretreated } \\
\text { Urine }\end{array}$ & Jantarat et al. (2021) \\
\hline Vancomycin & Fluorimetry & Fluorescent Probe & Tripeptide group & $0.1 \mu \mathrm{g} / \mathrm{ml}$ & $\begin{array}{l}0.02-100 \mu \mathrm{g} / \\
\mathrm{ml}\end{array}$ & $\begin{array}{l}\text { In vivo rabbits } \\
\text { blood }\end{array}$ & Mu et al. (2021) \\
\hline Ciprofloxacin & Fluorimetry & None & MIP & $6.86 \mu \mathrm{M}$ & $10-500 \mu \mathrm{M}$ & $\begin{array}{l}\text { Pre-treated } \\
\text { river water }\end{array}$ & Huang et al. (2021) \\
\hline Tetracycline & Fluorimetry & $\begin{array}{l}\text { N-Acetyl-L-Cysteine } \\
\text { capped Ag Nanoclusters }\end{array}$ & None & $0.47 \mu \mathrm{M}$ & $1.12-230 \mu \mathrm{M}$ & Milk & Zhang et al. (2021) \\
\hline D-Penicillamine & Fluorimetry & SiQDs & Ellman's Reagent & $0.48 \mu \mathrm{M}$ & $1-20 \mu \mathrm{M}$ & Human Serum & Liu et al. (2021b) \\
\hline Tetracycline & Fluorimetry & $\begin{array}{l}\text { Polynuclear Lanthanide } \\
\text { Metal Organic } \\
\text { Framework }\end{array}$ & None & $8 \mathrm{ng} / \mathrm{ml}$ & $\begin{array}{l}60 \mathrm{ng} / \mathrm{ml}- \\
10 \mu \mathrm{g} / \mathrm{ml}\end{array}$ & $\begin{array}{l}\text { Spiked Tap } \\
\text { Water }\end{array}$ & Li et al. (2021b) \\
\hline Tobramycin & Fluorimetry & PicoGreen & Aptamer & $21.86 \mathrm{nM}$ & $80 \mathrm{nM}-2 \mu \mathrm{M}$ & $\begin{array}{l}\text { Spiked Human } \\
\text { Serum }\end{array}$ & $\begin{array}{l}\text { Khajavian et al. } \\
\text { (2021) }\end{array}$ \\
\hline Epirubicin & Fluorimetry & $\begin{array}{l}\text { Nano-monoclinic copper- } \\
\text { tannic acid and Acridine } \\
\text { Orange }\end{array}$ & Guanine-rich ssDNA & $5.6 \mathrm{nM}$ & Up to $500 \mathrm{nM}$ & $\begin{array}{l}\text { Spiked Pre- } \\
\text { treated Urine }\end{array}$ & $\begin{array}{l}\text { Arunjegan et al. } \\
\text { (2021) }\end{array}$ \\
\hline Enrofloxacin & $\begin{array}{l}\text { Fibre-Optic } \\
\text { Fluorimetry }\end{array}$ & Coumarin & MIP Microsphere & $0.04 \mu \mathrm{M}$ & $\begin{array}{l}0.29-21.54 \\
\mu \mathrm{M}\end{array}$ & Sheep Serum & Carrasco et al. (2015) \\
\hline Hyaluronidase & Fluorimetry & CQD and Naphthalimide & Hyaluronic Acid & $0.09 \mathrm{U} / \mathrm{ml}$ & $0.1-16 \mathrm{U} / \mathrm{ml}$ & None & Raj et al. (2021) \\
\hline Posaconazole & Fluorimetry & & Aptamer & $0.32 \mu \mathrm{M}$ & & $10 \%$ Serum & $\begin{array}{l}\text { Wiedman et al. (2018) } \\
\text { (continued on next page) }\end{array}$ \\
\hline
\end{tabular}


Table 2 (continued)

\begin{tabular}{|c|c|c|c|c|c|c|c|}
\hline Analyte & Technique & Transducer & $\begin{array}{l}\text { Biorecognition } \\
\text { Element }\end{array}$ & LOD & $\begin{array}{l}\text { Detection } \\
\text { Range }\end{array}$ & $\begin{array}{l}\text { Validation } \\
\text { solution }\end{array}$ & Ref. \\
\hline & & $\begin{array}{l}\text { Reduced Graphene Oxide } \\
\text { and Fluorescein }\end{array}$ & & & $\begin{array}{l}0.32-0.40 \\
\mu \mathrm{M}\end{array}$ & & \\
\hline Methotrexate & Fluorimetry & CeTe QDs & MIP & $34 \mathrm{nM}$ & $0.5-20 \mu \mathrm{M}$ & $\begin{array}{l}\text { Pretreated } \\
\text { Human Plasma }\end{array}$ & Ensafi et al. (2017) \\
\hline Tamoxifen & Fluorimetry & Coumarin & MIP & Not Given & Not Given & None & Ray et al. (2016) \\
\hline Doxorubicin & Fluorimetry & $\begin{array}{l}\text { Europium-chelate } \\
\text { labelled competitive } \\
\text { antigen }\end{array}$ & Antibody & $3.8 \mathrm{ng} / \mathrm{ml}$ & $\begin{array}{l}3.8-3000 \mathrm{ng} / \\
\mathrm{ml}\end{array}$ & Human Serum & Liang et al. (2018) \\
\hline Doxorubicin & Fluorimetry & $\begin{array}{l}\text { Carbon dot-modified } \\
\text { silica gel }\end{array}$ & MIP & $13.8 \mathrm{nM}$ & $50-400 \mathrm{nM}$ & Spiked Plasma & Xu et al. (2017) \\
\hline Tetracycline & Fluorimetry & Thioflavin-T & $\begin{array}{l}\text { G-quadruplex } \\
\text { Aptamer }\end{array}$ & $1 \mathrm{nM}$ & $0.01-1 \mu \mathrm{M}$ & $\begin{array}{l}\text { Spiked Honey } \\
\text { and Milk }\end{array}$ & Dai et al. (2020) \\
\hline Salbutamol & Fluorimetry & CdTe QDs & MIP & $34 \mathrm{ng} / \mathrm{ml}$ & $0.1-25 \mathrm{ng} / \mathrm{ml}$ & $\begin{array}{l}\text { Animal Feed } \\
\text { and Meat } \\
\text { Samples }\end{array}$ & $\begin{array}{l}\text { Raksawong et al. } \\
\text { (2017) }\end{array}$ \\
\hline Tetracycline & Fluorimetry & $\begin{array}{l}\text { Fluorescein-modified } \\
\text { silica microspheres }\end{array}$ & MIP & $4.26 \mathrm{nM}$ & $4.26-150 \mathrm{nM}$ & $\begin{array}{l}\text { Human Serum } \\
\text { and Swine } \\
\text { Urine }\end{array}$ & Wang et al. (2018) \\
\hline Oxytetracycline & Fluorimetry & $\begin{array}{l}\text { MIL-101, Fluorescein } \\
\text { and enzyme-driven } \\
\text { exonuclease }\end{array}$ & Aptamer & $4.2 \mathrm{nM}$ & $\begin{array}{l}10 \mathrm{nM}-2.0 \\
\mu \mathrm{M}\end{array}$ & Duck Tissue & He et al. (2017) \\
\hline Ampicillin & Fluorimetry & Fluorescein and AuNPs & Aptamer & $20.6 \mathrm{nM}$ & $\begin{array}{l}100 \mathrm{nM}-100 \\
\mu \mathrm{M}\end{array}$ & Urine & Simmons et al. (2020) \\
\hline \multicolumn{8}{|c|}{ Surface Enhanced Raman Spectroscopy (SERS) } \\
\hline Kanamycin & SERS & $\begin{array}{l}2- \\
\text { Mercaptobenzothiazole } \\
\text { labelled } \mathrm{Au}+\mathrm{Ag} \text {-shell } \\
\text { NPs (+antibody) }\end{array}$ & $\begin{array}{l}\text { Antibody } \\
\text { functionalized } \\
\text { Hybrid } \mathrm{Fe}_{3} \mathrm{O}_{4} \mathrm{NPs}\end{array}$ & $2 \mathrm{pg} / \mathrm{ml}$ & $\begin{array}{l}2-80 \mathrm{pg} / \mathrm{ml}, \\
0.100-80 \mathrm{ng} / \\
\mathrm{ml}\end{array}$ & Pre-treated milk & Zengin et al. (2014) \\
\hline Flucytosine & SERS & Citrate-capped AgNPs & $\begin{array}{l}\text { Vertical Flow } \\
\text { Membrane }\end{array}$ & $222 \mathrm{ng} / \mathrm{ml}$ & $0-25 \mu \mathrm{g} / \mathrm{ml}$ & Serum & Berger et al. (2017) \\
\hline 5-Fluorouracil & SERS & Silver-doped sol gel & $\begin{array}{l}\text { Dihydropyrimidine } \\
\text { Dehydrogenase } \\
\text { (DPD) enzyme }\end{array}$ & $150 \mathrm{ng} / \mathrm{ml}$ & $2-100 \mu \mathrm{g} / \mathrm{ml}$ & Saliva & $\begin{array}{l}\text { Farquharson et al. } \\
(2005)\end{array}$ \\
\hline Ampicillin & SERS & Hydroxyamine-AgNPs & None & $27 \mathrm{ng} / \mathrm{ml}$ & $\begin{array}{l}100-600 \mathrm{ng} / \\
\mathrm{ml}\end{array}$ & None & El-Zahry et al. (2015) \\
\hline Penicillin G & & & & $29 \mathrm{ng} / \mathrm{ml}$ & $\begin{array}{l}100-600 \mathrm{ng} / \\
\mathrm{ml}\end{array}$ & & \\
\hline Carbenicillin & & & & $30 \mathrm{ng} / \mathrm{ml}$ & $\begin{array}{l}100-600 \mathrm{ng} / \\
\mathrm{ml}\end{array}$ & & \\
\hline Doxorubicin & SERS & $\begin{array}{l}\text { Modified L-cysteine, on } \\
\text { Au film on SiNP }\end{array}$ & None & $20 \mathrm{nM}$ & Up to $1.5 \mu \mathrm{M}$ & $\begin{array}{l}\text { Pretreated } \\
\text { serum }\end{array}$ & Panikar et al. (2020) \\
\hline Docetaxel & SERS & Ag@ZnO nanoparticles & None & $1 \mu \mathrm{g} / \mathrm{mL}$ & $\begin{array}{l}1-2000 \mu \mathrm{g} / \\
\mathrm{mL}\end{array}$ & $\begin{array}{l}\text { Pretreated rat } \\
\text { plasma }\end{array}$ & Xu et al. (2020) \\
\hline Paclitaxel & SERS & $\begin{array}{l}\text { Graphene oxide- } \\
\text { supported L-cysteine- }\end{array}$ & None & $15 \mathrm{nM}$ & $15-100 \mathrm{nM}$ & $\begin{array}{l}\text { Pretreated } \\
\text { blood serum }\end{array}$ & Panikar et al. (2019) \\
\hline Cyclophosphamide & & $\begin{array}{l}\text { functionalized starlike } \\
\text { gold nanoparticles }\end{array}$ & & $5 \mathrm{nM}$ & $5-100 \mathrm{nM}$ & $\begin{array}{l}\text { Pretreated } \\
\text { blood serum }\end{array}$ & \\
\hline Adalimumab & SERS & $\begin{array}{l}\text { Gold Coated Copper } \\
\text { Oxide }\end{array}$ & Modified TNF- $\alpha$ & $0.03 \mathrm{fM}$ & $\begin{array}{l}0.1 \mathrm{fM}-10 \\
\mathrm{nM}\end{array}$ & $\begin{array}{l}\text { Spiked human } \\
\text { plasma }\end{array}$ & Muneer et al. (2020a) \\
\hline Ciprofloxacin & FERS & None & None & $1.5 \mu \mathrm{M}$ & $2-14 \mu \mathrm{M}$ & None & Wolf et al. (2019) \\
\hline Methotrexate & SERS & Ag Colloids & None & $0.17 \mu \mathrm{M}$ & $0.2-2 \mu \mathrm{M}$ & None & Hidi et al. (2014) \\
\hline Cefuroxime & FERS & None & None & $7.5 \mu \mathrm{M}$ & $25-100 \mu \mathrm{M}$ & Urine & Yan et al. (2018) \\
\hline Berberine & SERS & $\begin{array}{l}\text { AgNPs in self-supporting } \\
\text { liquid film }\end{array}$ & None & $0.55 \mathrm{ppb}$ & $1-500 \mathrm{ppb}$ & $\begin{array}{l}\text { Spiked human } \\
\text { urine \& urine } \\
\text { samples }\end{array}$ & Liu et al. (2021a) \\
\hline Doxorubicin & SERS & $\begin{array}{l}\text { Quasi-three-dimensional } \\
\text { plasmonic nanostructure }\end{array}$ & None & $50 \mathrm{nM}$ & $0.05-2 \mu \mathrm{M}$ & $\begin{array}{l}\text { Protein-free } \\
\text { human plasma }\end{array}$ & Sun et al. (2016) \\
\hline Amitriptyline & & + poly (Carboxybetanine & & $0.05 \mu \mathrm{M}$ & Not reported & PBS & \\
\hline Carbamazepine & & acrylamide) & & $0.5 \mu \mathrm{M}$ & Not reported & PBS & \\
\hline Phenytoin & & & & $1 \mu \mathrm{M}$ & Not reported & PBS & \\
\hline Paclitaxel & SERS & $\begin{array}{l}\text { Microwave-treated Au- } \\
\text { polystyrene bead solid } \\
\text { film }\end{array}$ & None & $10 \mathrm{nM}$ & $10-100 \mathrm{nM}$ & Plasma & Yuen et al. (2010) \\
\hline Methotrexate & SERS & Au colloid & None & $2.2 \mu \mathrm{M}$ & Not reported & Urine & Subaihi et al. (2017b) \\
\hline Codeine & SERS & Ag NPs & None & $1.39 \mu \mathrm{M}$ & Not reported & $\begin{array}{l}\text { Spiked human } \\
\text { plasma }\end{array}$ & Subaihi et al. (2017a) \\
\hline Methotrexate & SERS & Au solid substrate & None & $0.1 \mu \mathrm{M}$ & $0.1-20 \mu \mathrm{M}$ & Serum & $\begin{array}{l}\text { Fornasaro et al. } \\
(2016)\end{array}$ \\
\hline 5- fluorouracil & SERS & Ag colloid & None & $2 \mu \mathrm{g} / \mathrm{mL}$ & $2-20 \mu \mathrm{g} / \mathrm{mL}$ & Sweat & Xing et al. (2018) \\
\hline Irinotecan & SERS & $\begin{array}{l}\text { Ag colloid coupled with } \\
\text { thin layer } \\
\text { chromatography }\end{array}$ & None & $2.73 \mu \mathrm{M}$ & Not reported & Serum & Vicario et al. (2015) \\
\hline Mitoxantrone & SERS & $\begin{array}{l}\mathrm{Au} \text { on } \mathrm{SiO}_{2} \text {-polymer } \\
\text { substrate }\end{array}$ & None & $\begin{array}{l}61.03 \mathrm{ng} / \\
\mathrm{mL}\end{array}$ & $\begin{array}{l}0.061-15.63 \\
\mu \mathrm{g} / \mathrm{mL}\end{array}$ & None & $\begin{array}{l}\text { Wu and Cunningham } \\
\text { (2014) }\end{array}$ \\
\hline Sunitinib & SERS & Klarite Au substrate & None & $18-26 \mathrm{ng}$ & 88-1872 ng & None & $\begin{array}{l}\text { Litti et al. (2016) } \\
\text { (continued on next page) }\end{array}$ \\
\hline
\end{tabular}


Table 2 (continued)

\begin{tabular}{|c|c|c|c|c|c|c|c|}
\hline Analyte & Technique & Transducer & $\begin{array}{l}\text { Biorecognition } \\
\text { Element }\end{array}$ & LOD & $\begin{array}{l}\text { Detection } \\
\text { Range }\end{array}$ & $\begin{array}{l}\text { Validation } \\
\text { solution }\end{array}$ & Ref. \\
\hline Irinotecan & & & & $60-70 \mathrm{ng}$ & $198-4032 \mathrm{ng}$ & & \\
\hline SN-38 & & & & $20-50 \mathrm{ng}$ & $107-1092$ ng & & \\
\hline 6-mercaptopurine & SERS & Ag NPs & None & $10 \mathrm{nM}$ & $0.01-10 \mu \mathrm{M}$ & Living cells & Fei et al. (2017) \\
\hline Methimazole & & & & $100 \mathrm{nM}$ & $\begin{array}{l}100 \mathrm{nM}-10 \\
\mathrm{mM}\end{array}$ & & \\
\hline 6-mercaptopurine & SERS & $\begin{array}{l}\beta \text {-Cyclodextrin } \\
\text { functionalized Ag colloid }\end{array}$ & None & $2.4 \mathrm{nM}$ & $4-20 \mathrm{nM}$ & None & Yang et al. (2015) \\
\hline Imatinib & SERS & $\begin{array}{l}\text { Silicon nanopillar coated } \\
\text { with } \mathrm{Ag}\end{array}$ & None & $0.43 \mu \mathrm{M}$ & Not reported & Spiked plasma & $\begin{array}{l}\text { Fornasaro et al. } \\
\text { (2018) }\end{array}$ \\
\hline 6-thioguanine & SERS & $\begin{array}{l}\text { Au colloid in microfluidic } \\
\text { system }\end{array}$ & None & $0.66 \mu \mathrm{M}$ & $1-10 \mu \mathrm{M}$ & $\begin{array}{l}\text { Spiked human } \\
\text { serum }\end{array}$ & Zhang et al. (2019) \\
\hline Erlotinib & SERS & $\begin{array}{l}\text { Laser-ablated Au NP } \\
\text { colloid }\end{array}$ & None & $300 \mathrm{nM}$ & $\begin{array}{l}300-3000 \\
\mathrm{nM}\end{array}$ & $\begin{array}{l}\text { Spiked human } \\
\text { plasma }\end{array}$ & Litti et al. (2019) \\
\hline Dexmedetomidine & SERS & Ag NPs on filter paper & None & $10 \mu \mathrm{g} / \mathrm{mL}$ & Not reported & $\begin{array}{l}\text { Spiked urine } \\
\text { samples and } \\
\text { spiked rat } \\
\text { serum }\end{array}$ & Han et al. (2020) \\
\hline $\begin{array}{l}\text { 3,4-methylenedioxy } \\
\text { methamphetamine }\end{array}$ & SERS & Au nanoparticles & None & $10 \mu \mathrm{M}$ & Not reported & None & Yan et al. (2017) \\
\hline $\begin{array}{l}\alpha \text {-methyltryptamine } \\
\text { hydrochloride }\end{array}$ & & & & $1 \mu \mathrm{M}$ & & & \\
\hline Sildenafil & SERS & $\mathrm{Ag}$ colloid & None & $2 \mathrm{mM}$ & $1-10 \mu \mathrm{g} / \mathrm{mL}$ & None & Zhao et al. (2017) \\
\hline Chloramphenicol & SERS & Au NPs colloid & None & $0.01 \mu \mathrm{M}$ & Not reported & None & Ding et al. (2017) \\
\hline 2-thiouracil & SERS & $\begin{array}{l}\text { Ag NPs seeded on } \\
\text { graphene nanosheets }\end{array}$ & None & $10 \mathrm{nM}$ & $0.1-1 \mu \mathrm{M}$ & None & Saleh et al. (2018) \\
\hline Chloroquine & SERS & $\mathrm{Ag}$ NPs colloid & None & $1 \mu \mathrm{M}$ & Not reported & None & Tackman et al. (2018) \\
\hline Doxycycline & & & & $10 \mu \mathrm{M}$ & & & \\
\hline Primaquine & & & & $0.5 \mu \mathrm{M}$ & & & \\
\hline Cocaine & SERS & $\begin{array}{l}\text { Hybrid Ag-Au } \\
\text { nanostructures }\end{array}$ & None & $10 \mu \mathrm{g} / \mathrm{mL}$ & Not reported & None & Gao et al. (2018a) \\
\hline Acyclovir & SERS & Ag NPs & None & $15 \mu \mathrm{M}$ & Not reported & None & Deng et al. (2019) \\
\hline Ceftriaxone & SERS & $\begin{array}{l}\text { Copper NPs in calcium } \\
\text { carbonate matrix }\end{array}$ & None & $0.52 \mathrm{pM}$ & $\begin{array}{l}0.1 \mu \mathrm{M}-0.1 \\
\mathrm{mM}\end{array}$ & None & Markina et al. (2018) \\
\hline Sulfadimethoxine & & & & $4.2 \mu \mathrm{M}$ & $10 \mu \mathrm{M}-1 \mathrm{mM}$ & & \\
\hline Cocaine & SERS & 2D Au NPs film & None & $500 \mathrm{ppb}$ & $1-50 \mathrm{ppm}$ & $\begin{array}{l}\text { Spiked human } \\
\text { urine }\end{array}$ & Meng et al. (2017) \\
\hline Levofloxacin & SERS & $\begin{array}{l}\text { Cotton blend fabric } \\
\text { modified with Ag NPs } \\
\text { and conductive inks }\end{array}$ & None & $1.0 \mathrm{mM}$ & Not reported & Artificial urine & Bindesri et al. (2018) \\
\hline Morphine & SERS & Au nanorods & None & $1 \mathrm{ppm}$ & $1-25 \mathrm{ppm}$ & $\begin{array}{l}\text { Spiked human } \\
\text { urine }\end{array}$ & Yu et al. (2018) \\
\hline Clozapine & SERS & Ag NPs & None & $0.1 \mu \mathrm{g} / \mathrm{mL}$ & $\begin{array}{l}0.5-50 \mu \mathrm{g} / \\
\mathrm{mL}\end{array}$ & $\begin{array}{l}\text { Spiked human } \\
\text { urine }\end{array}$ & Zhu et al. (2018) \\
\hline 6-thioguanine & SERS & $\begin{array}{l}\text { AuNPs embedded in } \\
\text { single-phase alginate } \\
\text { microparticles and Janus } \\
\text { microparticles }\end{array}$ & None & $0.1 \mu \mathrm{M}$ & $0.1-1 \mu \mathrm{M}$ & $\begin{array}{l}\text { Spiked human } \\
\text { serum }\end{array}$ & Yue et al. (2018) \\
\hline Cocaine & SERS & Au nanorods & None & $10 \mathrm{ng} / \mathrm{mL}$ & Not reported & $\begin{array}{l}\text { Spiked oral } \\
\text { fluid }\end{array}$ & D'Elia et al. (2018) \\
\hline $\begin{array}{l}\text { 2-fluoro- } \\
\text { methamphetamine }\end{array}$ & SERS & $\begin{array}{l}\text { Silver nanowire on silk } \\
\text { fibronin protein film }\end{array}$ & None & $500 \mu \mathrm{g} / \mathrm{mL}$ & Not reported & $\begin{array}{l}\text { Spiked } \\
\text { simulated } \\
\text { human sweat }\end{array}$ & Koh et al. (2021) \\
\hline Meropenem & SERS & $\begin{array}{l}\text { Flower-shaped gold } \\
\text { nanostructures on nickel } \\
\text { foam }\end{array}$ & None & 1 pM (LOQ) & $\begin{array}{l}1 \mathrm{pM}-0.5 \\
\mathrm{mM}\end{array}$ & $\begin{array}{l}\text { Spiked human } \\
\text { plasma }\end{array}$ & Muneer et al. (2020b) \\
\hline Glibenclamide & SERS & $\begin{array}{l}\text { Flower-like Ag particles } \\
\text { with MIPs }\end{array}$ & None & $<1 \mathrm{ng} / \mathrm{mL}$ & $\begin{array}{l}1 \mathrm{ng} / \mathrm{mL}- \\
100 \mu \mathrm{g} / \mathrm{mL}\end{array}$ & None & Ren and Li (2020) \\
\hline \multicolumn{8}{|c|}{ Chemiluminescence $\&$ bioluminescence } \\
\hline Gatifloxacin & Chemiluminescence & $\begin{array}{l}\text { AgNPs, Calcein and } \\
\mathrm{KMnO}_{4}\end{array}$ & None & $2.9 \mathrm{nM}$ & $8.9 \mathrm{nM}-4 \mu \mathrm{M}$ & Urine & $\begin{array}{l}\text { Wabaidur et al. } \\
\text { (2015a) }\end{array}$ \\
\hline Captopril & Chemiluminescence & AgNPs, $\mathrm{Br}^{-}$and $\mathrm{KMnO}_{4}$ & None & $0.12 \mathrm{nM}$ & $0.3-100 \mathrm{nM}$ & $\begin{array}{l}\text { Pre-treated and } \\
\text { diluted urine } \\
\text { and serum }\end{array}$ & Amjadi et al. (2013) \\
\hline Gemifloxacin & Chemiluminescence & $\begin{array}{l}\text { AgNPs, Luminol and } \mathrm{K}_{3} \\
{\left[\mathrm{Fe}(\mathrm{CN})_{6}\right]}\end{array}$ & None & $\begin{array}{l}2 \mathrm{pg} / \mathrm{ml}(\mathrm{S} / \\
\mathrm{N}=2)\end{array}$ & $\begin{array}{l}0.01-1000 \\
\mathrm{ng} / \mathrm{ml}\end{array}$ & $\begin{array}{l}\text { Urine, pre- } \\
\text { treated serum }\end{array}$ & Alarfaj et al. (2013) \\
\hline Naproxen & Chemiluminescence & $\begin{array}{l}\text { AgNPs, Eu(III)-sensitized } \\
\mathrm{Ce} \text { (IV) and } \mathrm{Na}_{2} \mathrm{~S}_{2} \mathrm{O}_{4}\end{array}$ & None & $0.11 \mathrm{ng} / \mathrm{ml}$ & $1-420 \mathrm{ng} / \mathrm{ml}$ & Diluted urine & $\begin{array}{l}\text { Kamruzzaman et al. } \\
\text { (2012) }\end{array}$ \\
\hline Moxifloxacin & Chemiluminescence & $\begin{array}{l}\text { AgNPs, Calcein and } \\
\mathrm{KMnO}_{4}\end{array}$ & None & $5 \mathrm{nM}$ & $\begin{array}{l}60 \mathrm{nM}-2.5 \\
\mu \mathrm{M}\end{array}$ & Urine & $\begin{array}{l}\text { Wabaidur et al. } \\
\text { (2015b) }\end{array}$ \\
\hline Nitrazepam & Chemiluminescence & $\begin{array}{l}\text { Rhodamine } 6 \mathrm{G} \text {, AgNPs, } \\
\mathrm{K}_{3}(\mathrm{FN})_{6}\end{array}$ & None & $100 \mathrm{pM}$ & $\begin{array}{l}1.0 \mathrm{nM}-10 \\
\mu \mathrm{M}\end{array}$ & $\begin{array}{l}\text { Pretreated and } \\
\text { diluted urine } \\
\text { and plasma }\end{array}$ & Han et al. (2014) \\
\hline Cefditoren Pivoxil & Chemiluminescence & $\begin{array}{l}\text { AgNPs, Luminol and } \mathrm{K}_{3} \\
{\left[\mathrm{Fe}(\mathrm{CN})_{6}\right]}\end{array}$ & None & $0.5 \mathrm{pg} / \mathrm{ml}$ & $\begin{array}{l}1 \mathrm{pg} / \mathrm{ml}-5 \\
\mu \mathrm{g} / \mathrm{ml}\end{array}$ & $\begin{array}{l}\text { Urine, pre- } \\
\text { treated and } \\
\text { diluted serum }\end{array}$ & Alarfaj et al. (2015) \\
\hline
\end{tabular}


Table 2 (continued)

\begin{tabular}{|c|c|c|c|c|c|c|c|}
\hline Analyte & Technique & Transducer & $\begin{array}{l}\text { Biorecognition } \\
\text { Element }\end{array}$ & LOD & $\begin{array}{l}\text { Detection } \\
\text { Range }\end{array}$ & $\begin{array}{l}\text { Validation } \\
\text { solution }\end{array}$ & Ref. \\
\hline $\begin{array}{l}\text { Methotrexate } \\
\text { Tacrolimus } \\
\text { Sirolimus } \\
\text { Cyclosporine A } \\
\text { Topiramate } \\
\text { Digoxin }\end{array}$ & Bioluminescence & Luciferase & Receptor Protein & $\begin{array}{l}\text { Below } \\
\text { therapeutic } \\
\text { range }\end{array}$ & $\begin{array}{l}\text { Covers } \\
\text { therapeutic } \\
\text { range }\end{array}$ & Patient serum & Griss et al. (2014) \\
\hline $\begin{array}{l}\text { Gentamicin } \\
\text { Tobramycin }\end{array}$ & Bioluminescence & $\begin{array}{l}\text { T3 } 3_{\text {pro:NLuc DNA + T3 }} \\
\text { RNAP mRNA + IVTT } \\
\text { reagents }\end{array}$ & None & $\begin{array}{l}0.22 \mu \mathrm{g} / \mathrm{mL} \\
\text { Not } \\
\text { reported }\end{array}$ & $\begin{array}{l}0-0.8 \mu \mathrm{g} / \mathrm{mL} \\
\text { Not reported }\end{array}$ & $\begin{array}{l}\text { Spiked human } \\
\text { serum }\end{array}$ & Matsuura et al. (2019) \\
\hline Amikacin & & & & $\begin{array}{l}\text { Not } \\
\text { reported }\end{array}$ & Not reported & & \\
\hline Trastuzumab & Bioluminescence & $\begin{array}{l}\text { Luciferase NanoLuc } \\
\text { connected via a }\end{array}$ & $\begin{array}{l}\text { Antibody-binding } \\
\text { epitope sequence }\end{array}$ & $0.16 \mu \mathrm{M}$ & $\begin{array}{l}31 \% \text { dynamic } \\
\text { range }\end{array}$ & None & $\begin{array}{l}\text { van Rosmalen et al. } \\
\text { (2018) }\end{array}$ \\
\hline Rituximab & & $\begin{array}{l}\text { semiflexible linker to a } \\
\text { green fluorescent }\end{array}$ & flanking the linker & $3.9 \mu \mathrm{M}$ & $\begin{array}{l}22 \% \text { dynamic } \\
\text { range }\end{array}$ & & \\
\hline Obinutuzumab & & $\begin{array}{l}\text { acceptor protein } \\
\text { mNeonGreen }\end{array}$ & & $0.23 \mathrm{nM}$ & $\begin{array}{l}26 \% \text { dynamic } \\
\text { range }\end{array}$ & & \\
\hline Cetuximab & & & & $55 \mathrm{nM}$ & $\begin{array}{l}60 \% \text { dynamic } \\
\text { range }\end{array}$ & & \\
\hline Methotrexate & Bioluminescence & $\begin{array}{l}\text { Antibody fragments } \\
\text { fused to NanoLuc }\end{array}$ & $\begin{array}{l}\text { Antibody Fab } \\
\text { fragment }\end{array}$ & $\begin{array}{l}\text { Not } \\
\text { reported }\end{array}$ & $\begin{array}{l}53 \mathrm{nM}-0.13 \\
\mathrm{mM}\end{array}$ & $\begin{array}{l}\text { Spiked human } \\
\text { serum }\end{array}$ & Xue et al. (2017) \\
\hline Theophylline & & luciferase and ANAP-tag & & & $\begin{array}{l}0.84 \\
\mu \mathrm{M}-0.93 \mathrm{mM}\end{array}$ & & \\
\hline Quinine & & & & & $\begin{array}{l}0.49 \\
\mu \mathrm{M}-0.59 \mathrm{mM}\end{array}$ & & \\
\hline $\begin{array}{l}\text { Imipramine } \\
\text { Promazine }\end{array}$ & Chemiluminescence & $\begin{array}{l}\text { Mesoporous TiO2-Ru } \\
\text { (bpy) })_{3}^{2+} \text { NPs }\end{array}$ & None & $\begin{array}{l}0.1 \mathrm{pM} \\
0.5 \mathrm{pM}\end{array}$ & $1-100 \mathrm{pM}$ & None & $\begin{array}{l}\text { Al-Hetlani et al. } \\
\text { (2018) }\end{array}$ \\
\hline Paracetamol & Chemiluminescence & $\mathrm{KMnO}_{4}+$ rhodamine-6G & None & $78 \mathrm{nM}$ & $\begin{array}{l}0.12 \\
\mu \mathrm{M}-0.185 \\
\mathrm{mM}\end{array}$ & $\begin{array}{l}\text { Spiked human } \\
\text { urine }\end{array}$ & Emdadi et al. (2021) \\
\hline $\begin{array}{l}\text { Methapyrilene } \\
\text { Imipramine }\end{array}$ & Chemiluminescence & $\begin{array}{l}\mathrm{Ru}(\mathrm{bpy})_{3}^{2+} \text { complex } \\
\text { electrostatically linked to } \\
\text { poly (MAA-co-EDMA) } \\
\text { monolith }\end{array}$ & None & $\begin{array}{l}6.8 \mathrm{pM} \\
8.2 \mathrm{pM}\end{array}$ & $5-50 \mathrm{pM}$ & None & $\begin{array}{l}\text { Al-Hetlani et al. } \\
(2020)\end{array}$ \\
\hline Metronidazole & Chemiluminescence & $\begin{array}{l}\text { Cerium doped magnetite } \\
\text { nanoparticles + luminol- } \\
\mathrm{K}_{3} \mathrm{Fe}(\mathrm{CN})_{6}\end{array}$ & None & $0.391 \mu \mathrm{M}$ & $\begin{array}{l}3.47 \\
\mu \mathrm{M}-93.7 \mu \mathrm{M}\end{array}$ & $\begin{array}{l}\text { Spiked human } \\
\text { serum }\end{array}$ & Orooji et al. (2020) \\
\hline Bambuterol & Chemiluminescence & $\mathrm{Ru}(\mathrm{bpy})_{3}^{2+}$ luminophore & None & $1.48 \mathrm{nM}$ & $10 \mathrm{nM}-2 \mu \mathrm{M}$ & None & Halawa et al. (2021) \\
\hline Pefloxacin & Photoluminescence & $\begin{array}{l}\text { Red-emissive Carbon } \\
\text { Dots }\end{array}$ & None & $0.85 \mu \mathrm{M}$ & Up to $900 \mu \mathrm{M}$ & None & Li et al. (2021a) \\
\hline Doxorubicin & Chemiluminescence & $\begin{array}{l}\text { Nitrogen doped } \\
\text { Graphene QDs }\end{array}$ & MIP & $4.7 \mathrm{ng} / \mathrm{ml}$ & $\begin{array}{l}20-260 \mathrm{ng} / \\
\mathrm{ml}\end{array}$ & $\begin{array}{l}\text { Spiked Human } \\
\text { Serum }\end{array}$ & $\begin{array}{l}\text { Amjadi and Jalili } \\
(2016)\end{array}$ \\
\hline Tamoxifen & Chemiluminescence & $\begin{array}{l}\text { Mn(IV) with } \\
\text { formaldehyde }\end{array}$ & MIP & $40 \mathrm{ng} / \mathrm{ml}$ & $\begin{array}{l}100 \mathrm{ng} / \mathrm{ml}- \\
1 \mu \mathrm{g} / \mathrm{ml}\end{array}$ & $\begin{array}{l}\text { Pretreated } \\
\text { Human Urine }\end{array}$ & Nie et al. (2005) \\
\hline Sulfasalazine & $\begin{array}{l}\text { Solid-Phase } \\
\text { Luminescence }\end{array}$ & CdSeS/ZnS QDs & MIP & $7.1 \mathrm{nM}$ & $0.02-1.5 \mu \mathrm{M}$ & $\begin{array}{l}\text { Pretreated } \\
\text { Human Plasma } \\
\text { and Urine }\end{array}$ & $\begin{array}{l}\text { Ahmadpour and } \\
\text { Hosseini (2019) }\end{array}$ \\
\hline \multicolumn{8}{|c|}{ Surface Plasmon Resonance (SPR) } \\
\hline Amikacin & SPR & Gold chip & $\begin{array}{l}\text { Anti-Amikacin } \\
\text { Antibody }\end{array}$ & $0.13 \mathrm{ng} / \mathrm{ml}$ & $\begin{array}{l}0.33-5.77 \\
\mathrm{ng} / \mathrm{ml}\end{array}$ & None & $\begin{array}{l}\text { Losoya-Leal et al. } \\
\text { (2015) }\end{array}$ \\
\hline Tobramycin & SPR & Gold chip & Tobramycin aptamer & $\begin{array}{l}0.625 \mu \mathrm{g} / \\
\mathrm{ml}(\mathrm{LOQ})\end{array}$ & Not given & Patient serum & Tenaglia et al. (2018) \\
\hline Infliximab & SPR & Gold & $\begin{array}{l}\text { Anti-IFX monoclonal } \\
\text { antibody }\end{array}$ & $1.00 \mathrm{ng} / \mathrm{ml}$ & $\begin{array}{l}\sim 3-100 \mathrm{ng} / \\
\mathrm{ml}\end{array}$ & $\begin{array}{l}\text { Dried Blood } \\
\text { Spots, diluted } \\
\text { patient serum, } \\
\text { plasma and } \\
\text { whole blood }\end{array}$ & Lu et al. (2017) \\
\hline Infliximab (IFX), & SPR & $\begin{array}{l}\text { Alginate-based polymer } \\
\text { matrix bound to gold }\end{array}$ & $\mathrm{TNF} \alpha$ & $\begin{array}{l}0.20 \mu \mathrm{g} / \mathrm{ml} \\
\text { (LOQ) }\end{array}$ & $0.5-8 \mu \mathrm{g} / \mathrm{ml}$ & $\begin{array}{l}\text { Pre-treated and } \\
\text { diluted serum }\end{array}$ & Beeg et al. (2019) \\
\hline IFX antibodies (ATI) & & surface & IFX & $\begin{array}{l}2.5 \mu \mathrm{g} / \mathrm{ml} \\
(\mathrm{LOQ})\end{array}$ & $5-40 \mu \mathrm{g} / \mathrm{ml}$ & & \\
\hline Amikacin & SPR & Gold SPR chip & MIP film & $2.5 \mathrm{ng} / \mathrm{ml}$ & $\begin{array}{l}0.01-0.15 \\
\mu \mathrm{g} / \mathrm{ml}\end{array}$ & $\begin{array}{l}\text { Pre-treated and } \\
\text { diluted plasma }\end{array}$ & Yola et al. (2014) \\
\hline Ciprofloxacin & SPR (+SPRi) & Gold SPR chip & MIP & $\sim 80 \mathrm{pg} / \mathrm{ml}$ & $\begin{array}{l}10 \mathrm{pM}-100 \\
\mathrm{nM}\end{array}$ & None & Luo et al. (2016) \\
\hline Methotrexate & LSPR & $\begin{array}{l}\text { Folic Acid functionalized } \\
\text { AuNPs }\end{array}$ & $\begin{array}{l}\text { human dihydrofolate } \\
\text { reductase (hHDFR) }\end{array}$ & $155 \mathrm{nM}$ & $155-360 \mathrm{nM}$ & $\begin{array}{l}\text { Pre-treated, } \\
\text { diluted human } \\
\text { Serum }\end{array}$ & Zhao et al. (2012) \\
\hline Methotrexate & LSPR & $\begin{array}{l}\text { Folic Acid functionalized } \\
\text { AuNPs }\end{array}$ & $\begin{array}{l}\text { Human Dihydrofolate } \\
\text { Reductase (hHDFR) }\end{array}$ & $28 \mathrm{nM}$ & $28-500 \mathrm{nM}$ & $\begin{array}{l}\text { Pre-treated, } \\
\text { diluted human } \\
\text { Serum }\end{array}$ & Zhao et al. (2015) \\
\hline $\begin{array}{l}\text { Morphine-3- } \\
\text { Glucuronide }\end{array}$ & SPR & BIAcore chips & $\begin{array}{l}\text { Polyclonal } \\
\text { Antibodies }\end{array}$ & $\begin{array}{l}762 \mathrm{pg} / \mathrm{ml} \\
\text { (LOQ) }\end{array}$ & $\begin{array}{l}762-24,400 \\
\mathrm{pg} / \mathrm{ml}\end{array}$ & Diluted Urine & Dillon et al. (2003) \\
\hline $\begin{array}{l}\text { Melagatran } \\
\text { Phenytoin }\end{array}$ & $\begin{array}{l}\text { LSPR } \\
\text { SPR }\end{array}$ & Gold nanorods & Human $\alpha$ - Thrombin & $\begin{array}{l}0.9 \mathrm{nM} \\
<50 \mathrm{nM}\end{array}$ & $\begin{array}{l}0.9-25 \mathrm{nM} \\
\text { Not reported }\end{array}$ & Serum & $\begin{array}{l}\text { Guo et al. (2012) } \\
\text { FU et al. (2007) }\end{array}$ \\
\hline
\end{tabular}


Table 2 (continued)

\begin{tabular}{|c|c|c|c|c|c|c|c|}
\hline Analyte & Technique & Transducer & $\begin{array}{l}\text { Biorecognition } \\
\text { Element }\end{array}$ & LOD & $\begin{array}{l}\text { Detection } \\
\text { Range }\end{array}$ & $\begin{array}{l}\text { Validation } \\
\text { solution }\end{array}$ & Ref. \\
\hline & & $\begin{array}{l}\text { BSA-Phenytoin } \\
\text { conjugate on Au layer }\end{array}$ & $\begin{array}{l}\text { Antiphenytoin } \\
\text { antibody }\end{array}$ & & & $\begin{array}{l}\text { Pre-treated } \\
\text { saliva }\end{array}$ & \\
\hline Captopril & SPR & AgNP & $\begin{array}{l}\text { Dispersive Liquid- } \\
\text { Liquid } \\
\text { Microextraction }\end{array}$ & $0.51 \mathrm{nM}$ & $\begin{array}{l}0.77-22.50 \\
\mathrm{nM}\end{array}$ & $\begin{array}{l}\text { Pre-treated and } \\
\text { diluted serum, } \\
\text { diluted urine }\end{array}$ & Hashemi et al. (2018) \\
\hline Lidocaine $\mathrm{HCl}$ & $\begin{array}{l}\text { Plasmon Resonance } \\
\text { Scattering }\end{array}$ & $\begin{array}{l}\text { Homocysteine } \\
\text { functionalized AgNPs, } \\
\text { plus Cu(II) }\end{array}$ & $\begin{array}{l}\mathrm{Cu}(\mathrm{II}) \text { and } \\
\text { homocysteine }\end{array}$ & $4.6 \mathrm{nM}$ & $50-500 \mathrm{nM}$ & $\begin{array}{l}\text { Urine, pre- } \\
\text { treated and } \\
\text { diluted dosed } \\
\text { rat's blood }\end{array}$ & Dou et al. (2013) \\
\hline Ceftazidime & LSPR & $\begin{array}{l}\text { Functionalized gold } \\
\text { nanoparticles modified } \\
\text { optical fibers }\end{array}$ & $\begin{array}{l}\text { Bacteriolysis } \\
\text { signatures of } \\
\text { P. aeruginosa }\end{array}$ & $10 \mathrm{ng} / \mathrm{mL}$ & $\begin{array}{l}0.01-1 \mu \mathrm{g} / \\
\mathrm{mL}\end{array}$ & $\begin{array}{l}\text { Spiked human } \\
\text { serum }\end{array}$ & Nag et al. (2020) \\
\hline Paraoxon & Leaky Waveguide & $\begin{array}{l}\text { Titanium and Silicon } \\
\text { layers }\end{array}$ & $\begin{array}{l}\text { Acetylcholinesterase } \\
\text { enzyme }\end{array}$ & $6 \mathrm{nM}$ & Not given & None & $\begin{array}{l}\text { Zourob and Goddard } \\
(2005)\end{array}$ \\
\hline Irinotecan & SPR & $\begin{array}{l}\text { Streptavidin-coated } \\
\text { Carboxymethyl dextran } \\
\text { chip }\end{array}$ & Aptamer & $55 \mathrm{ng} / \mathrm{ml}$ & $\begin{array}{l}0.1-7.5 \mu \mathrm{g} / \\
\mathrm{ml}\end{array}$ & $\begin{array}{l}\text { Filtered Human } \\
\text { Plasma }\end{array}$ & Puscasu et al. (2021) \\
\hline Imatinib & SPR & $\begin{array}{l}\text { Streptavidin-coated } \\
\text { Carboxymethyl dextran } \\
\text { chip }\end{array}$ & Aptamer & $79.5 \mathrm{ng} / \mathrm{ml}$ & $0.4-6 \mu \mathrm{g} / \mathrm{ml}$ & $\begin{array}{l}\text { Filtered Human } \\
\text { Plasma }\end{array}$ & Tartaggia et al. (2021) \\
\hline Acenocoumarol & LSPR & Gold Nanodisk & Antibody & $0.66 \mathrm{nM}$ & $3.24-777 \mathrm{nM}$ & None & $\begin{array}{l}\text { Peláez-Gutierrez et al. } \\
\text { (2017) }\end{array}$ \\
\hline Naproxen & LSPR & AuNPs & $\begin{array}{l}\text { Thiolated } \\
\beta \text {-cyclodextran }\end{array}$ & $0.6 \mathrm{ng} / \mathrm{ml}$ & $4-180 \mathrm{ng} / \mathrm{ml}$ & $\begin{array}{l}\text { Pretreated } \\
\text { Spiked Urine } \\
\text { and Wastewater }\end{array}$ & $\begin{array}{l}\text { Khodaveisi et al. } \\
\text { (2017) }\end{array}$ \\
\hline Doxycycline & SPR & AuNPs & $\begin{array}{l}\text { Protease-Activated } \\
\text { Receptor-1 }\end{array}$ & $7 \mathrm{pM}$ & $\begin{array}{l}0.1 \mathrm{nM}-100 \\
\mu \mathrm{M}\end{array}$ & None & Kazmi et al. (2020) \\
\hline Bromocriptine & SPR & $\begin{array}{l}\text { Carboxymethyl dextran } \\
\text { chip }\end{array}$ & Laccase Enzyme & $1 \mathrm{pg} / \mathrm{ml}$ & $\begin{array}{l}1 \mathrm{pg} / \mathrm{ml}-1 \\
\mu \mathrm{g} / \mathrm{ml}\end{array}$ & $\begin{array}{l}\text { Diluted } \\
\text { Simulated } \\
\text { Blood }\end{array}$ & Jabbari et al. (2017) \\
\hline Adalimumab & Fibre-Optic SPR & AuNPs & Antibody & $1 \mu \mathrm{g} / \mathrm{ml}$ & $1-16 \mu \mathrm{g} / \mathrm{ml}$ & Patient Serum & Bian et al. (2018) \\
\hline Digoxin & LSPR & AuNPs & Antibody & $2 \mathrm{ng} / \mathrm{ml}$ & $\begin{array}{l}\text { Up to } 200 \\
\mathrm{ng} / \mathrm{ml}\end{array}$ & $\begin{array}{l}\text { Fetal Bovine } \\
\text { Serum }\end{array}$ & $\begin{array}{l}\text { Nikfarjam et al. } \\
\text { (2017) }\end{array}$ \\
\hline $\begin{array}{l}\text { Enrofloxacin } \\
\text { Sulfapyridine } \\
\text { Chloramphenicol }\end{array}$ & SPR & Gold Chip & Antibody & $\begin{array}{l}0.30 \mathrm{ng} / \mathrm{ml} \\
0.29 \mathrm{ng} / \mathrm{ml} \\
0.26 \mathrm{ng} / \mathrm{ml}\end{array}$ & Not Given & $\begin{array}{l}\text { Diluted Spiked } \\
\text { Milk Samples }\end{array}$ & $\begin{array}{l}\text { Fernández et al. } \\
\text { (2010) }\end{array}$ \\
\hline Neomycin B & SPR & Gold Chip & RNA Aptamer & $5 \mathrm{nM}$ & $\begin{array}{l}10 \mathrm{nM}-100 \\
\mu \mathrm{M}\end{array}$ & None & $\begin{array}{l}\text { de-los-Santos-Álvarez } \\
\text { et al. (2009) }\end{array}$ \\
\hline Chloramphenicol & SPR & $\begin{array}{l}\text { Gold }+ \text { Chromium } \\
\text { Layers, with immobilized } \\
\text { BSA-Chloramphenicol }\end{array}$ & Antibody & $0.5 \mathrm{ng} / \mathrm{ml}$ & Not given & $\begin{array}{l}\text { Pretreated Meat } \\
\text { Samples }\end{array}$ & Dong et al. (2009) \\
\hline $\begin{array}{l}\text { Neomycin } \\
\text { Kanamycin } \\
\text { Streptomycin }\end{array}$ & SPR & $\begin{array}{l}\text { Imprinted AuNP, } \\
\text { functionalized with } \\
\text { thioaniline and } \\
\text { (mercaptophenyl) } \\
\text { boronic groups }\end{array}$ & $\begin{array}{l}\text { Boronic Acid Ligand } \\
+ \text { MIP }\end{array}$ & $\begin{array}{l}2 \mathrm{pM} \\
1 \mathrm{pM} \\
200 \mathrm{fM}\end{array}$ & $\begin{array}{l}2 \mathrm{pM}-20 \mathrm{nM} \\
1 \mathrm{pM}-1 \mathrm{nM} \\
\text { Not given }\end{array}$ & $\begin{array}{l}\text { Diluted Milk } \\
\text { Samples }\end{array}$ & Frasconi et al. (2010) \\
\hline Infliximab & SPR & Gold Chip & Antibody & $23.5 \mathrm{ng} / \mathrm{ml}$ & Not given & Human Serum & Zeni et al. (2020) \\
\hline
\end{tabular}

require no pre-treatment procedure and to be highly sensitive $(0.06 \mathrm{mg}$ $\mathrm{L}^{-1}$ for $100 \mu \mathrm{L}$ of sample), it failed to detect deferiprone in real EBC samples. As such, it is clear that beyond fabricating sensitive and selective optical biosensors, the choice of sample matrix is critical as drug concentrations in different biological fluids can differ by orders of magnitude. Correlation between serum and alternative biofluid drug concentration, and with the drug's pharmacodynamics, must also be well-established for these state-of-the-art sensors to applied in clinical practice.

As with spectrophotometry, fluorescence-based drug quantification can also be achieved with the combination of fluorescent probes and aptamers for enhanced selectivity. One example is the detection of tetracycline using a triple helix molecular switch aptamer, where the aptamer has two arm segments and the signal transduction probe has a quencher and a fluorescent probe at either end (Fig. 3A) (Jalalian et al., 2015). Fluorescence intensity was measured using a Synergy H4 microplate reader at $520 \mathrm{~nm}$. This approach achieved an LOD of $8.48 \mathrm{nM}$ in rat serum diluted with sodium phosphate buffer and demonstrated moderate selectivity. Specifically, the aptasensor was weakly and non-responsive to doxycycline and clindamycin respectively. Given that tetracycline and doxycycline are structurally very similar yet distinct antibiotics, an approximately 6-fold lower fluorescence intensity observed with doxycycline in comparison to tetracycline would, in our opinion, warrant further improvements in the choice of aptamer to enhance accuracy.

\subsection{Raman spectroscopy}

Raman spectroscopy is a qualitative and quantitative chemical analysis technique based on Raman scattering (Jones et al., 2019). When light interacts with and excites a molecule, it is scattered either elastically (i.e. the scattered and incident photon possess the same energy) or inelastically (i.e. the scattered and incident photon have different energy). The former is known as Rayleigh scattering and occurs predominantly, while the latter is known as Raman scattering. After Raman scattering occurs, the molecule is left in a different rovibronic state compared to its ground state. For the total energy of the system to be kept constant, the scattered photons possess different energy levels compared to the incident photon, and therefore different frequency and wavelengths. This difference is equal to the energy difference between the rovibronic state and initial ground state of the molecule. Detailed discussion on the mechanism of Raman scattering is outside the scope of this review, and can instead be found here (Jones et al., 2019). In a typical Raman spectroscopy setup, laser light is incident upon the 
A

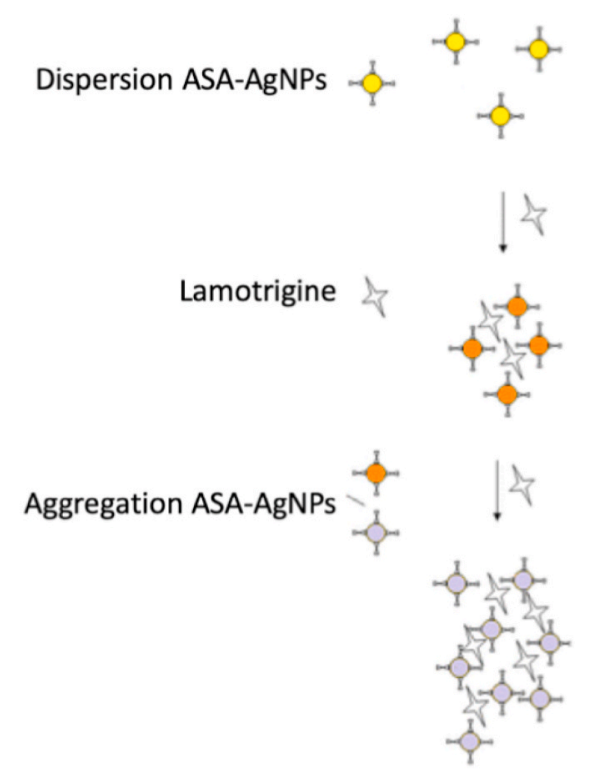

B

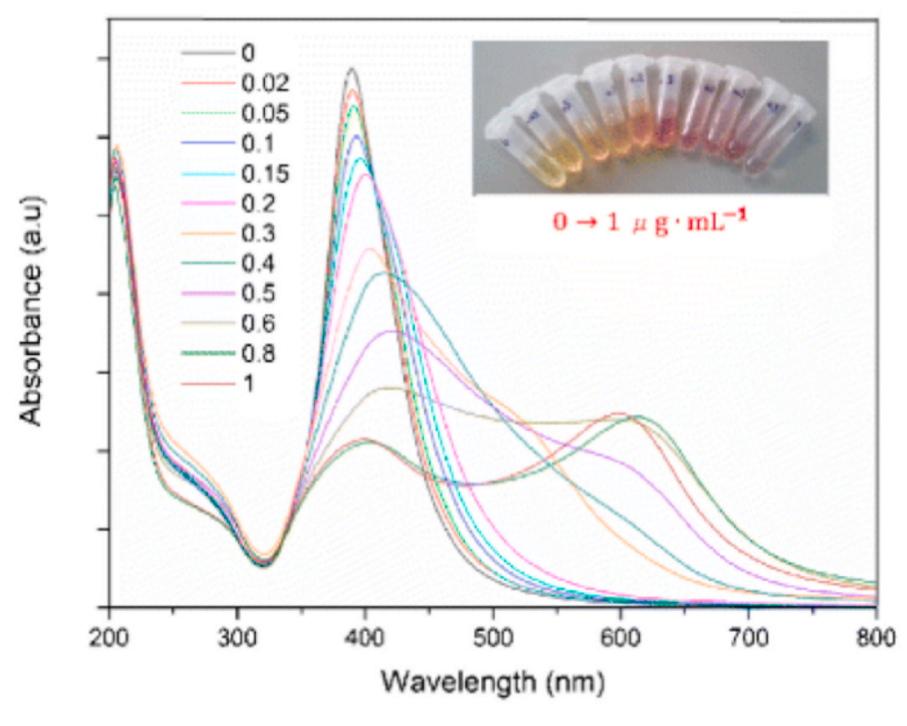

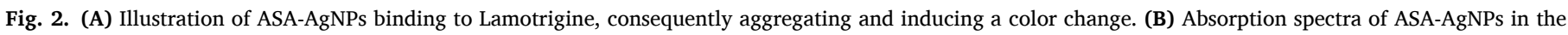

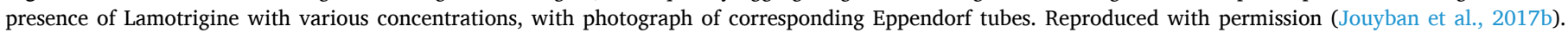
Copyright (C) 2017, Springer Nature. (For interpretation of the references to color in this figure legend, the reader is referred to the Web version of this article.)

sample through objective lenses. The scattered light subsequently passes through an edge pass or notch filter that blocks Rayleigh-scattered laser light, after which it is focused and directed to a spectrometer. The spectrometer measures the frequency and wavelengths of the scattered light, producing a spectrum that shows a pattern of peaks unique to the molecule. Each peak occurs at the specific wavelength position of the Raman scattered light, and corresponds to a specific molecular bond vibration (e.g. individual bonds such as $\mathrm{C}-\mathrm{O}, \mathrm{C}=\mathrm{C}, \mathrm{C}-\mathrm{H}$ etc.) or groups of bonds (e.g. benzene rings). The intensity of the peaks is directly proportional to the concentration of the analyte, and can therefore be calibrated and used for TDM purposes.

Typically, Raman scattering gives very weak signals. However, these can be enhanced by using rough metal surfaces which amplify the signal near their surface, giving Surface Enhanced Raman Spectroscopy. Additionally, the signal can be enhanced by containing the sample and the excitation laser within an optical hollow fiber, thereby increasing the interaction between light and analyte and producing a stronger Raman signal; this is Fiber-Enhanced Raman Spectroscopy (FERS) (Wang et al., 2017a). Here, fiber adaptors are often fitted onto both ends of the optical fiber; these contain inlet ports through which the sample can be injected, and waste can be ejected. Laser light is focused through objective lenses and coupled into the fiber core through an optical window. The backscattered light passes through the same optical window and subsequently follows conventional Raman spectroscopy setups as described above.

Because SERS measurements generate an array of peaks, there is the potential for the spectra to be interpreted using computer algorithms to improve the accuracy of the measurement. One study that took advantage of this was for the detection of docetaxel, with AgNPs anchored to ZnONPs as the SERS substrate (Xu et al., 2020). In this study, the Raman spectra were recorded using a SPLD-RAMAN-785-Q Spectrometer equipped with a $785 \mathrm{~nm}$ diode laser. Several different computer algorithms were compared to develop pattern recognition models. It was found that using competitive-adapted reweighted sampling with back propagation artificial neural network with AdaBoost (CARS BP-AdaBoost) was able to detect docetaxel in rat serum with recoveries of $83.06-115.76 \%$ with RSD $\leq 5.74 \%$. While this is limited to serum, and does have a large range for recoveries, it does demonstrate the opportunity that machine learning and artificial intelligence pose for TDM.

In addition to this, some studies have focused on using SERS for TDM. One example of this is the detection of cephalosporin antibiotics in urine (Markina and Markin, 2019). Here, urine samples were pre-treated with aluminum hydroxide gel, which removed intrinsic urine components such as urea and creatinine, and their $\mathrm{pH}$ were subsequently adjusted (Fig. 4A). Pre-treatment served to minimize background signals caused by intrinsic urine components. Using hydroxylamine stabilized AgNPs as the SERS substrate and a "high-performance modular Raman system" from Ocean Optics, the LOQs achieved were between 19 and $290 \mu \mathrm{g} / \mathrm{ml}$, with RSD values not exceeding the normal SERS values of $\sim 15-20 \%$. In another study, the detection of adalimumab in spiked diluted human plasma using SERS was demonstrated using a gold-coated copper oxide substrate functionalized with TNF- $\alpha$ (Muneer et al., 2020a). Interestingly, this study used a handheld Raman spectrophotometer (ID Raman mini 2, Ocean Optics) with a spectral resolution of $12 \mathrm{~cm}^{-1}$, and managed to achieve an LOD of $0.030 \mathrm{fM}$ (RSD = $5.7 \%$ ) in water. When tested in spiked human blood plasma, the relationship between Raman intensity at $934 \mathrm{~cm}^{-1}$ and adalimumab concentration were highly linear $\left(\mathrm{R}^{2}=0.999\right)$, and an average $98.8 \%$ agreement between SERS and ELISA quantifications was achieved. The detection of erlotinib with cysteamine and sulfo-SANPAH functionalized AuNP colloids was also demonstrate in spiked plasma, with an LOQ of $300 \mathrm{nM}$ using a Renishaw InVia $\mu$ Raman spectrometer (Litti et al., 2019). This was done with competitive binding against the highly SERS active molecule propynyl fluorescent red (Fig. 4B). This method demonstrates a plausible strategy for detecting analytes that give a weak/no SERS signal.

FERS has also drawn interest in its application in drug detection and quantification due to its high sensitivity. For instance, FERS was used to quantify cefuroxime in synthetic urine and spiked urine samples from healthy volunteers (Yan et al., 2018). The optical setup in this study comprised a hollow sensor fiber that was filled with urine sample through a custom-made fiber adaptor. Laser light with excitation wavelengths of $532 \mathrm{~nm}$ and $835 \mathrm{~nm}$ were coupled to the fiber core through an objective lens. The backscattered light was spatially filtered with a pinhole, and the Raman spectra was subsequently acquired with 
A
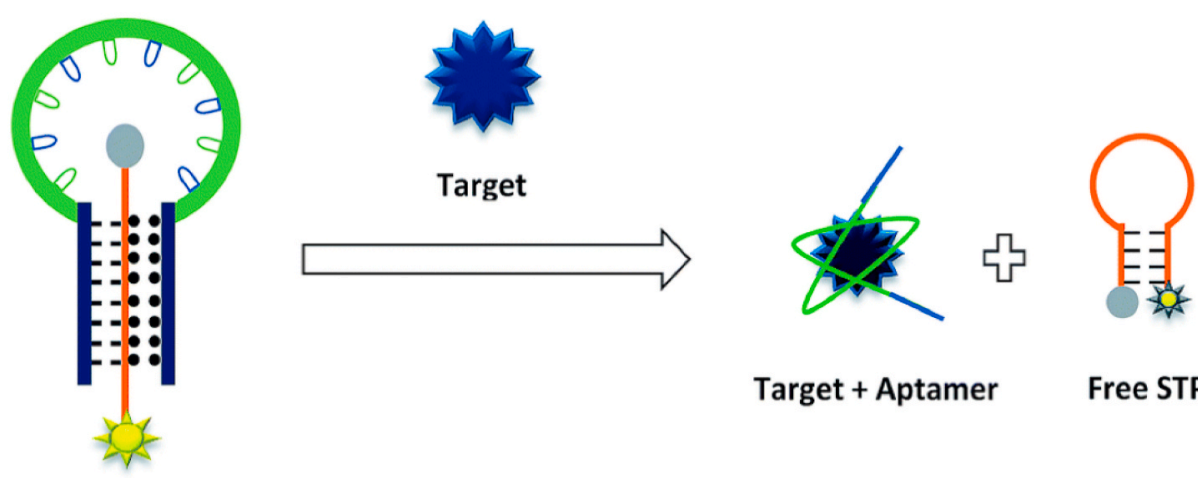

Target + Aptamer

Free STP

THMS

B

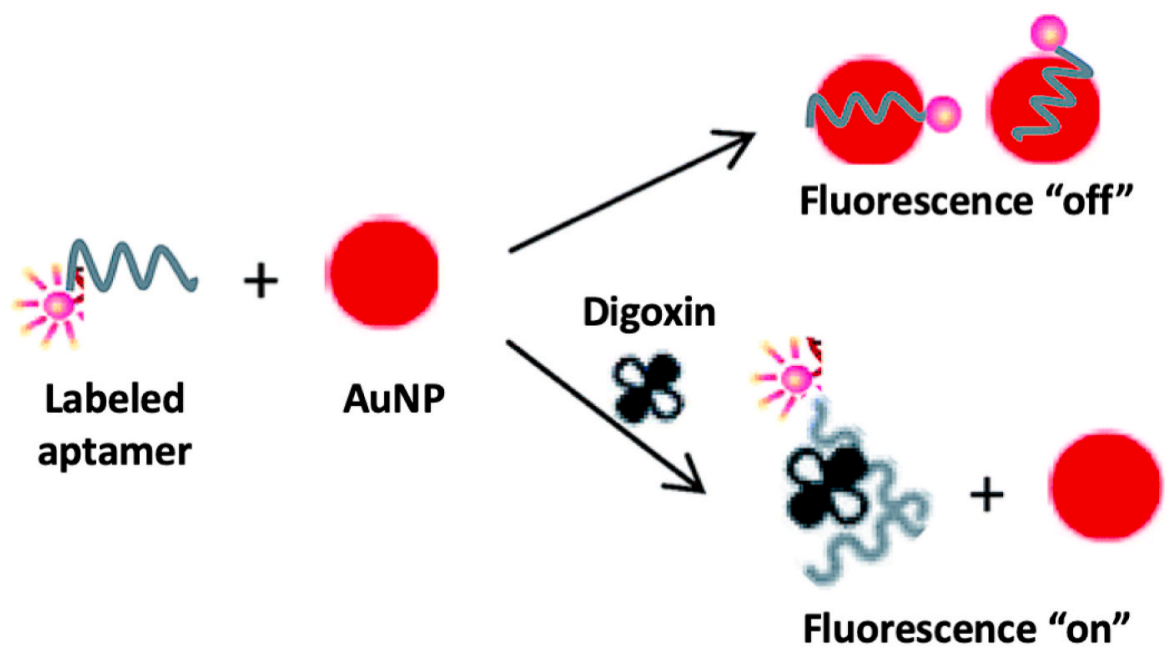

Fig. 3. (A) Schematic diagram of triple helix molecular switch (THMS) aptamer. In the initial state, the aptamer binds to the signal transduction probe (STP) (orange strand), forming a gap between the fluorophore and the quencher. This results in a fluorescence signal. Introducing the target molecule into the system causes the THMS to disassemble, releasing the STP to form a stem loop structure via intramolecular DNA hybridization. This quenches the fluorescence emission intensity. Reproduced with permission (Jalalian et al., 2015). Copyright @ 2015, RSC Publishing. (B) Schematic diagram of fluorescence quenching aptasensor, in which addition of digoxin induces the formation of aptamer-digoxin conjugate. The aptamer is released from the surface of the AuNPs, resulting in a fluorescent signal. Reproduced with permission (Sarreshtehdar Emrani et al., 2015). Copyright $\odot$ 2015, RSC Publishing. (For interpretation of the references to color in this figure legend, the reader is referred to the Web version of this article.)

an Isoplane SCT-320 spectrometer. The measured intensity of the Raman peak at $1485 \mathrm{~cm}^{-1}$ showed strong linear correlation with cefuroxime concentration in both synthetic urine and spiked human urine at $532 \mathrm{~nm}$ and $835 \mathrm{~nm}$ respectively (Fig. 4C). An excitation wavelength of $835 \mathrm{~nm}$ was used for spiked human urine due to strong fluorescence background observed at an excitation wavelength of $532 \mathrm{~nm}$. FERS was shown to perform significantly better than conventional Raman sensing: for quantifying cefuroxime in synthetic urine, FERS improved the LOD by 66.7-fold (from $0.5 \mathrm{mM}$ using conventional Raman to $7.5 \mu \mathrm{M}$ using FERS), the LOQ by 66.8-fold (from $1.67 \mathrm{mM}$ using conventional Raman to $25 \mu \mathrm{M}$ using FERS), and required only $104 \mathrm{~nL}$ of sample as opposed to $1 \mathrm{~mL}$ in conventional Raman. Similar levels of enhancement were observed with spiked human urine samples. FERS measurements of cefuroxime concentration in urine samples from healthy volunteers $6 \mathrm{~h}$ and $9 \mathrm{~h}$ after oral intake of cefuroxime also showed high levels of agreement with measurements obtained using HPLC. These results validate the use of FERS for fast, ultra-sensitive and accurate quantification of cefuroxime in human urine.

\subsection{Chemiluminescence}

Chemiluminescence sensors measures the production of light from a chemical reaction. This can be enhanced using metals or nanoparticles
(Aboul-Enein et al., 1999; Han et al., 2014). Bioluminescence is a form of chemiluminescence, using the ability of natural species to produce and emit light following a chemical reaction. One of the most common bioluminescent species are the luciferase enzymes, which react with a luciferin to emit light. This property can be used for sensing through bioluminescent resonance energy transfer (BRET), where the energy from the bioluminescent compound is used to excite a fluorophore, decreasing the signal from the bioluminescent species and increasing the signal from the fluorescent compound (Berthold Technologies, 2020).

Another study highlighting the adaptability of optical biosensors was done using bioluminescent sensor proteins (BSP). These consisted of a receptor protein, a luciferase enzyme, and a synthetic molecule with a fluorophore and ligand for the receptor protein (Griss et al., 2014). The binding of the receptor protein to its ligand causes the fluorophore to be brought close to the bioluminescent enzyme, permitting BRET between them (Fig. 5A). Therefore, in its native state, the bioluminescent sensor proteins produce a dominant fluorescent signal (red). However, when an analyte that binds to the receptor protein is added, the ligand is displaced from the receptor protein. BRET efficiency between the fluorophore and bioluminescent enzyme consequently decreases, causing the bioluminescent signal (blue) to increase and fluorescence (red) to decrease.

This system was used to detect methotrexate, tacrolimus, sirolimus, 
A

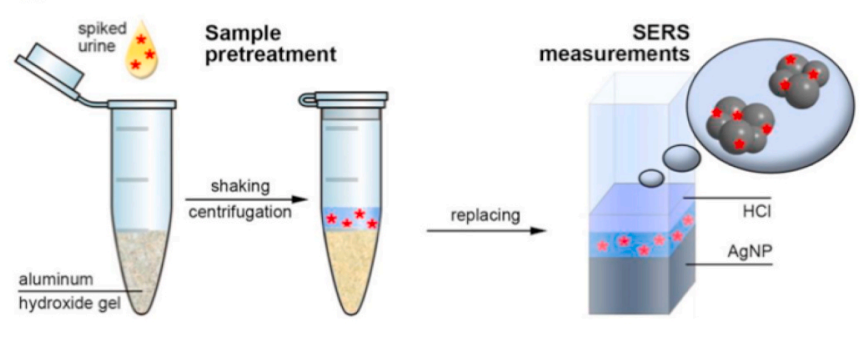

B

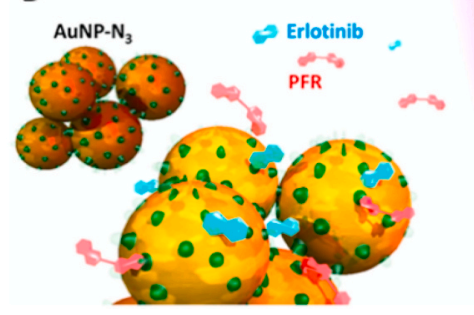

C

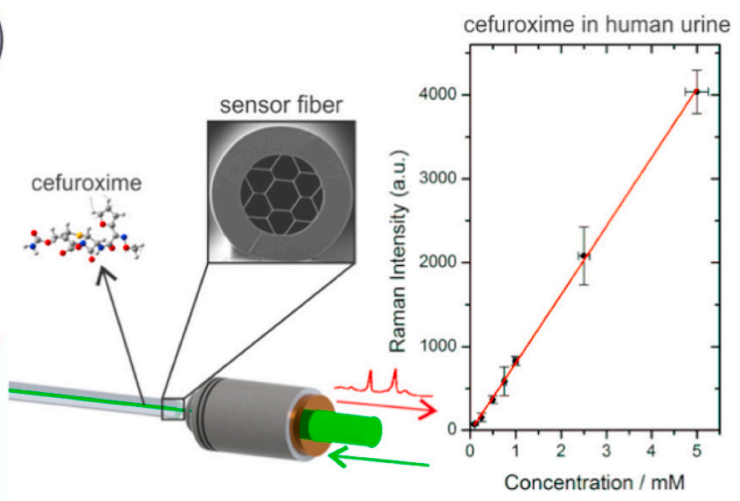

Fig. 4. (A) Scheme of pre-treating urine with aluminum hydroxide gel and subsequent SERS measurement. Reproduced with permission (Markina and Markin, 2019). Copyright @ 2019, MDPI. (B) Illustration of erlotinib and propynyl fluorescent red (PFR) competing for azide group on functionalized AuNPs. SERS signal produce by PFR is much greater than that or erlotinib, therefore increasing erlotinib concentrations result in decreasing intensity of PFR signal. Reproduced with permission (Litti et al., 2019). Copyright $\odot$ 2018, Elsevier Inc. (C) Illustration of FERS used to quantify cefuroxime, with calibration curve inserted. Reproduced with permission (Yan et al., 2018). Copyright $\odot$ 2018, American Chemical Society. (For interpretation of the references to color in this figure legend, the reader is referred to the Web version of this article.)

A

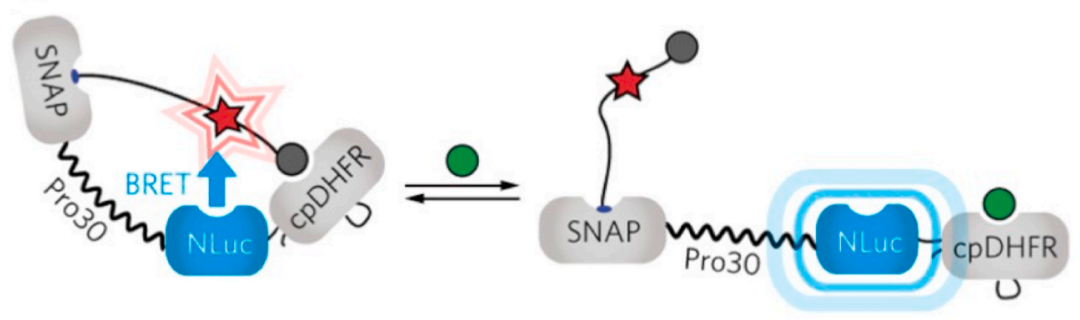

B

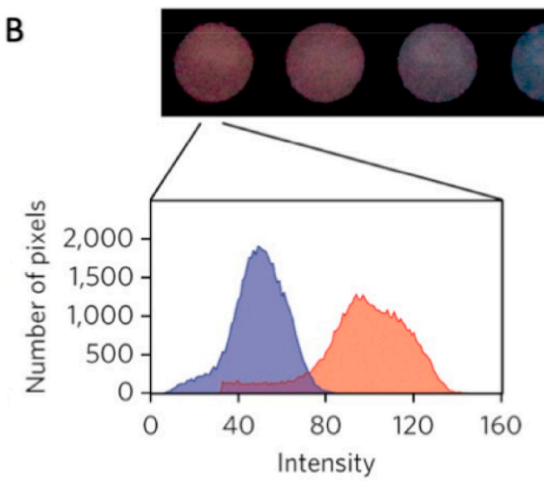

C
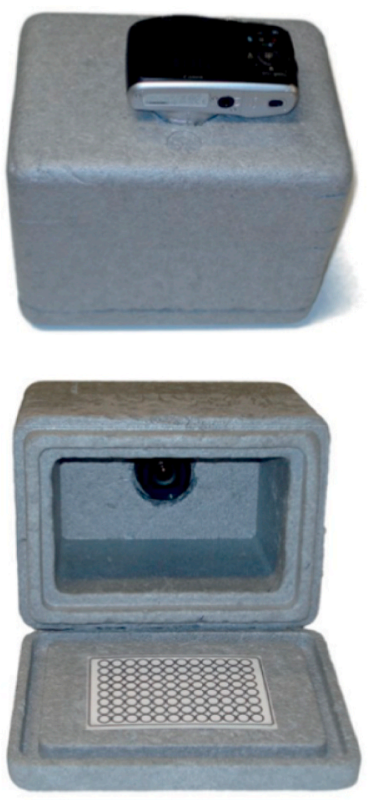

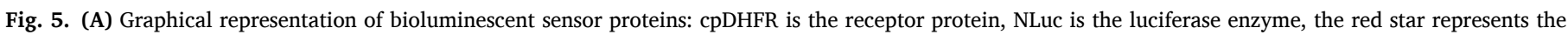

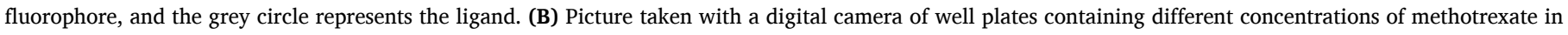

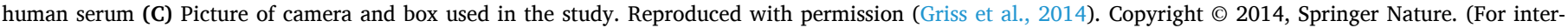
pretation of the references to color in this figure legend, the reader is referred to the Web version of this article.)

cyclosporine A, topiramate and digoxin. The BSPs for this were similar, as only the receptor protein and ligand needed to be changed in order to detect the different drugs. Furthermore, the use of different ligands allowed for tunable detection ranges. This was implemented using a point-and-shoot camera (Canon PowerShot SX150 IS digital camera) and a box containing chromatography paper in a well plate (Fig. 5B and
C). For image analysis, a java servlet based on ImageJ was used to construct red and blue histograms, from which the weighted mean was calculated and used as the average content of red and blue. Using this setup, samples from patients undergoing methotrexate treatment were tested. The bioluminescent method achieved a high correlation with traditional fluorescent polarization immunoassay $\left(R^{2}=0.995\right)$, over a 
wide concentration range $(\sim 100 \mathrm{nM}-10 \mu \mathrm{M})$. While the technique did require some sample preparation, it clearly highlights the applicability of optical sensors to measure different therapeutic agents.

\subsection{Surface Plasmon Resonance (SPR)}

Surface Plasmon Resonance (SPR) uses surface plasmons (oscillations of the free electrons confined to the interface of a metal and dielectric) to monitor the changes in refractive index near a surface. Light is shone onto a surface and reflects at different angles; each angle has a corresponding refractive index associated with it. The surface plasmons are excited at particular energies depending on the refractive indices of the two surfaces, and so these angles of light are absorbed, giving a reduction in the intensity of the reflected light. By changing the refractive index, the angle for the minimum changes and can consequently be monitored (Tang et al., 2010). This technique can be taken further if a metal coated nanostructure is used which gives strong transmission of light through it. This is known as Transmission Surface Plasmon Resonance (TSPR), in which the transmission of light is measured to determine the change in refractive index (Lertvachirapaiboon et al., 2018). Alternatively, if the surface plasmons are generated in a nanoparticle which is much smaller than the wavelength of light, they are highly localized to the nanoparticle. This gives rise to Localized Surface Plasmon Resonance (LSPR) (Fong and Yung, 2013). Further, an SPR device can achieve a higher throughput by using a 2D array to give SPR imaging (SPRi) (Guner et al., 2017). If the light is scattered, and not just absorbed, then the technique is labelled as plasmon resonance scattering. Finally, if a dielectric film is used instead of a metal layer, an evanescent wave is formed instead of a plasmon, and the sensing is termed a Leaky Waveguide (Gupta and Goddard, 2020).

SPR has been used for the detection of drugs in plasma. One example of this is 6-aminopenicillanic acid, where 4-formyl pyridinium propylthioacetate stabilized silver nanoparticles (ThPy-AgNPs) were used (Anwar et al., 2019). Detection in human plasma did not show significant changes in the decrease in the SPR band caused by ThPy-AgNPs, demonstrating the applicability of SPR for this. In addition, infliximab (IFX) was detected using fiber optic SPR using anti-IFX antibodies and AuNPs conjugated to detection antibodies (Lu et al., 2017). This was shown to be applicable in diluted whole blood, dried blood spots and IFX treated patients, where, compared to ELISA, Pearson correlation coefficients of 0.997 were achieved. Further, the detection of acenocoumarol using gold nanodisks and an indirect competitive antibody immunoassay was demonstrated in diluted plasma (Fig. 6B), with recoveries of 90.5-103.5\% (Peláez et al., 2018). Another study quantified lidocaine using homocysteine coated AuNPs with a recovery of $94.75 \%$ in spiked urine, with an RSD of $4.1 \%$ (Dou et al., 2013).

The potential for SPR to be used in multiplex measurements has also been demonstrated. One key illustration of this is the simultaneous detection of infliximab and the anti-IFX antibody (Beeg et al., 2019). These were detected using a microfluidic chip with tumor necrosis factor $\alpha$ (ligand for infliximab detection) and infliximab (ligand for the antibody detection) immobilized onto flow channels. By comparing the SPR shift from the different ligand strips to the control strips, the concentration of both analytes could be quantified. This was then tested in acid pre-treated patient serum and compared to a traditional ELISA detection; the infliximab concentrations showed a good correlation, but the relationship for the antibody was less clear. It was suggested this was because of an underestimation by the ELISA system. In addition, the simultaneous measurement of the antibiotics ciprofloxacin and azithromycin was demonstrated using MIPs, where both could be detected simultaneously, although the specificity of the azithromycin MIP was not as high (Luo et al., 2016).

\section{Seeing the bigger picture: the strengths \& limitations of optical biosensors}

\subsection{Strengths of optical biosensors}

Optical biosensors offer an assortment of advantages that continue to spur research into their application in TDM. Foremost, optical biosensors boast high sensitivity, having been demonstrated to be capable of measuring analytes in femtomolar concentrations (Muneer et al., 2020a). Such sensitivity makes optical biosensors suitable for measuring serum concentrations of highly potent drugs that are administered in very small doses, such as levothyroxine (dose $\sim 50-100 \mu \mathrm{g}$ ). Electrochemical biosensors have also been lauded for their apparent sensitivity (Pollard et al., 2021), though a literature-based comparison between the two general types of biosensors would not be fair given the differing parameters employed in each study. Nevertheless, a recent study pitted electrochemical and chemiluminescent biosensors against each other using identical protocols, and found that chemiluminescence-based biosensors generally yielded "slightly lower LOD values and wider dynamic ranges" (Roda et al., 2020). Therefore, the high sensitivity of optical biosensors makes them ideal devices for detecting drugs in clinically relevant concentrations.

Certainly, the performance of optical biosensors may be impaired when deployed in an open environment as opposed to stringent
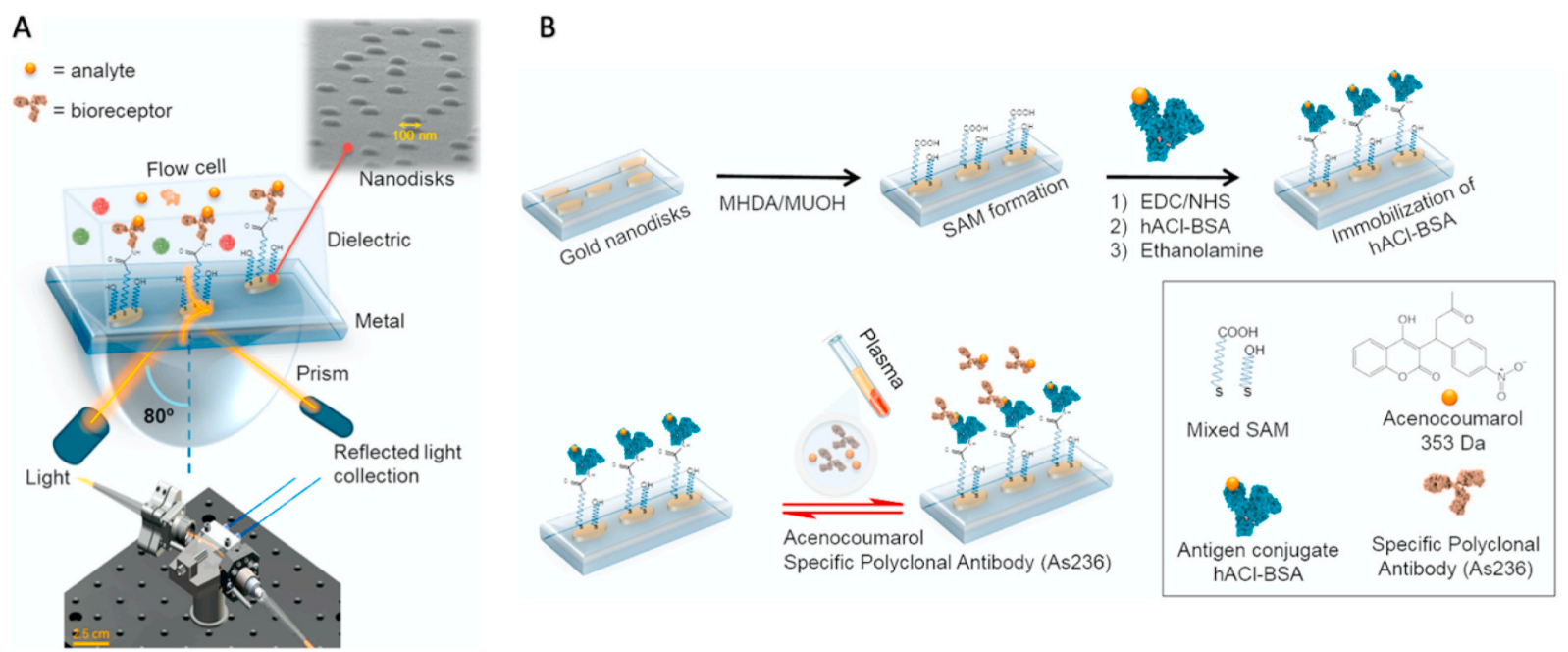

Fig. 6. (A) Illustration of nanoplamonic biosensor. (B) Graphical scheme of biofunctionalization protocol (top) and indirect competitive antibody immunoassay (bottom). Reproduced with permission (Peláez et al., 2018). Copyright $\odot$ 2018, Elsevier B.V. 

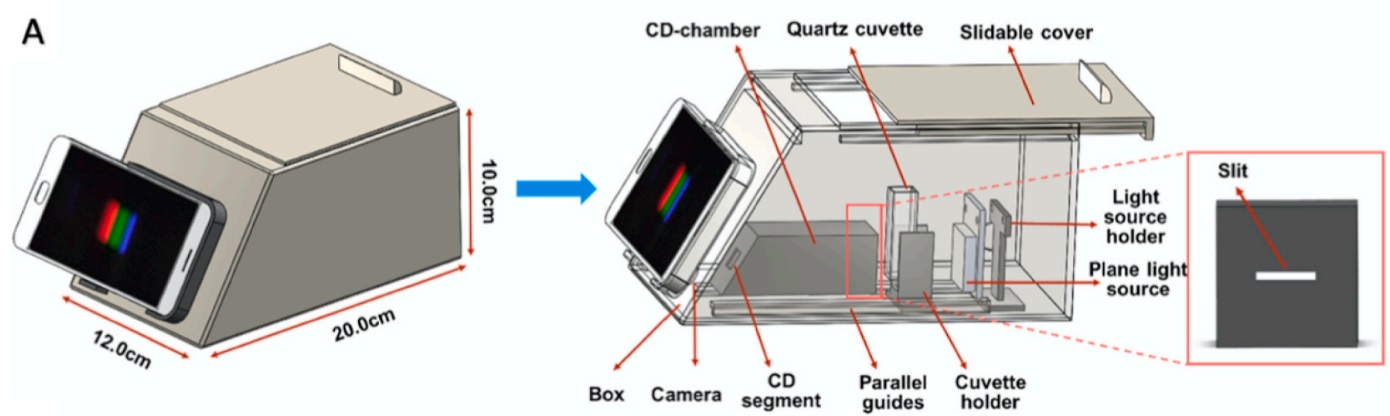

B

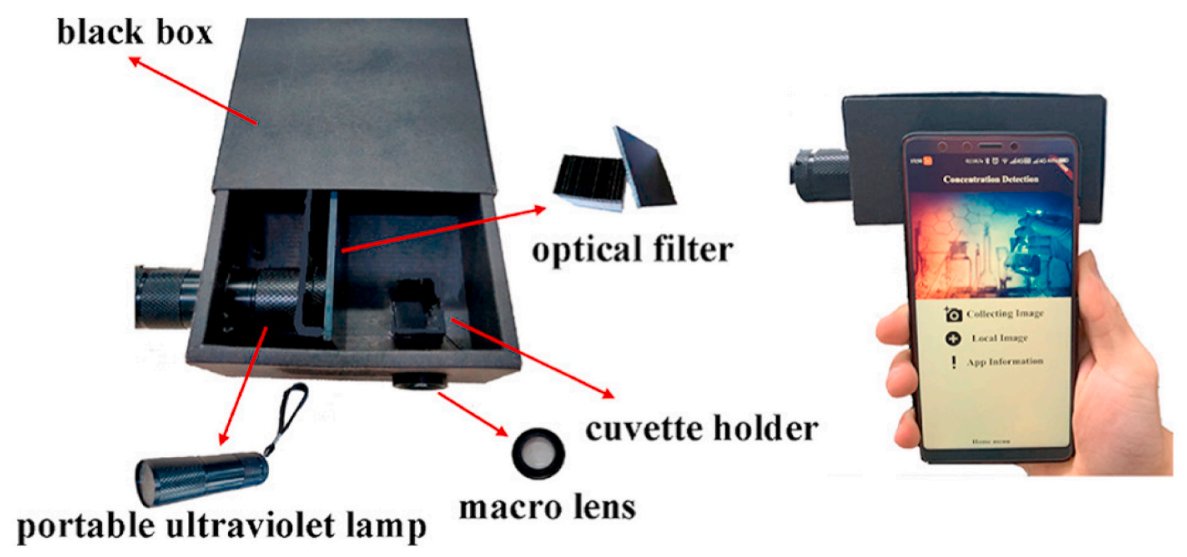

Fig. 7. (A) Schematic of a smartphone-based CD-spectrometer for colorimetric detection. Reproduced with permission (Kong et al., 2020). Copyright $\odot$ 2019, Elsevier B.V. (B) Structure of portable smartphone-based fluorescence detector. Reproduced with permission (Rao et al., 2020). Copyright $\odot$ 2020, American Chemical Society.

experiment conditions. It is therefore fortunate that optical biosensors can be easily integrated with microfluidic structures, providing a closed and relatively stable biosensing environment to recover or perhaps even enhance sensitivity (Liao et al., 2019). Microfluidic chips confer additional benefits, such as increased efficiency through multi-analyte detection, reduced sample volumes, and portability. These features support the development of on-site optical biosensors capable of providing clinicians with actionable data for swift dose adjustments. This is made further possible with recent studies exploring the use of smartphone-based fluorometers \& spectrophotometers (Fig. 7) (Kong et al., 2020). Integration with smartphones also enhances data transmission and analysis through cloud computing and efficient algorithms, whilst also providing a graphical user interface that makes the overall system user-friendly. These obviate the use of expensive laboratory-grade equipment, consequently reducing the overall operational cost of the device. Therefore, optical biosensors could be made affordable and accessible to majority of patients, enabling their widespread adoption. This is especially the case for label-free biosensors, which benefit from additional reductions to material cost. Admittedly, there has been criticism over the loose use of the phrase "low cost" in describing emerging sensors in scientific communications. Estimating the expected purchase price of sensors requires "complicated financial considerations that are beyond the scope of academic prototype development" (Wilson et al., 2019). Indeed, while we can be hopeful that these sensors might eventually become affordable products, we should bear in mind that these are merely postulation and do not warrant excessive zeal.

Finally, optical biosensors are inherently capable of simultaneously measuring multiple analytes, also known as multiplexing. This is possible by measuring different wavelength signals that are unique to each individual analyte of interest (Emmerson et al., 2010). Not only does multiplexing improve the efficiency of optical biosensors, but it can also be used to quantify an internal standard for self-calibration, thereby improving the sensor's long-term accuracy and reliability.

\subsection{Technical challenges}

As with all emerging technology, adoption of optical biosensors in clinical practice is impeded by numerous technical and regulatory challenges. Addressing the outstanding technical limitations is paramount to increasing the odds of regulatory approval, but does not guarantee it. Ultimately, optical biosensors must produce accurate and reproducible data, be sufficiently robust, and ideally operate noninvasively.

However, at present, the reliability, repeatability and stability of optical biosensors is not regularly evaluated in their respective articles. Stability is a particularly important aspect for label-based sensors that employ organic biorecognition elements. Given the harsh environmental conditions of some biological samples (e.g. $\mathrm{pH}$ of saliva and blood), protein-based molecules such as enzymes and antibodies may denature and lose their conformational shape and functionality. Fluctuations in storage conditions, such as temperature and humidity, can also gradually induce denaturation through hydrolysis, oxidation or thermal denaturation. While these may be addressed by imposing strict storage conditions, such arrangements are not ideal since enforcing a cold chain increases transport and maintenance costs, consequently hindering adoption in resource-limited regions. The performance of aptamers is also often influenced by $\mathrm{pH}$, ionic strength and temperature, which could result in discrepancies between results obtained from laboratory and clinical samples (Charbgoo et al., 2016). Sensor degradation is a source of systematic error that must be mitigated to achieve acceptable analytical precision, quantified by the coefficient of variation (CV). For bioanalytical assays such as TDM, CVs must not exceed $15 \%$, though it is conceivable that these limits should be even lower for the quantification of narrow therapeutic drugs (Ates et al., 2020). As such, optical biosensor must be robust to changes in these conditions. Amongst the minority of studies that have evaluated the stability of their sensors, testing protocols are not standardized, with differing storage or stress conditions and duration. Indeed, current sensors and materials do not 
generally demonstrate adequate stability over time to make them suitable for clinical deployment, even if they were meant to be single-use. This is further compounded by a lack of consensus over a standard of stability for these sensors to meet. An agreement over both the stability testing protocol and standard of stability must be established between regulatory authorities and the biosensors community.

Apart from biorecognition elements, synthetic nanomaterials used in the fabrication of optical biosensors also pose various challenges. Similarly, the stability of nanoparticles is a concern as they can aggregate over time or leach into and contaminate biofluids. For instance, silver nanoparticles have been observed to be prone to oxidation in wet environments (e.g. biofluids), which consequently leads to poor adhesion to and release from surfaces (Bhalla et al., 2019). Gold nanoparticles (AuNPs) are prone to salt-induced aggregation, which consequently results in unstable and unreliable signals (Charbgoo et al., 2016). Conjugating nanoparticles onto biorecognition elements to serve as labels can also impair the latter's target recognition efficiency and binding properties, resulting in some preference for label-free sensors (Charbgoo et al., 2016). From a practical standpoint, nanofabrication processes are usually neither cost-effective nor scalable, although advances in materials science might eventually resolve this issue.

Optical biosensors are also less time responsive compared to their electrochemical counterparts as a period of incubation is often necessary (Roda et al., 2020). They are consequently unsuitable for continuous monitoring of drug concentrations, which can cause crucial time step information needed for accurate pharmacokinetic calculations to be missed. As such, it might not be feasible to integrate an optical biosensor into a closed-loop feedback system, which is perhaps the ideal strategy to maximize the time in therapeutic range. Rather, considering their faster turnover compared to conventional analytical techniques (i.e. LC-MS/MS) and their high sensitivity, optical biosensors are better suited for frequent point-of-care applications.

\subsection{Regulatory challenges}

The healthcare sector is notoriously resistant to change, owing to extensive regulatory guidelines and long-standing clinical practices. Certainly, these are necessary to safeguard patient safety, but they are also major hinderances to obtaining regulatory approval and achieving widespread adoption of optical biosensors.

In 1992, regulatory bodies from the European Union, United States, Canada, Australia and Japan formed the Global Harmonization Task Force (GHTF) with the aim of achieving "greater uniformity between national medical device regulatory systems" (International Medical Device Regulators Forum). As of 2012, the GHTF has been superseded by the International Medical Device Regulators Forum (IMDRF). The GHTF published the Principles of Medical Devices Classification, which prescribes rules that classify medical devices into 4 groups, where Class A are the least hazardous and Class D the most hazardous (The Global Harmonization Task Force, 2012). In accordance to Rule 10(i), optical biosensors for TDM purposes are likely categorized as Class B. Optical biosensors designed to quantify drugs where variations in its serum concentration can pose immediate danger to the patient, such as tacrolimus (an immunosuppressant used to lower the risk of organ rejection), will warrant a Class $C$ categorization. Therefore, for the sensor to reach the market, the manufacturer must implement and maintain an effective Quality Management System. This includes a process to assess the device's conformity to the Essential Principles of Safety and Performance of Medical Devices published by the IMDRF (IMDRF Good Regulatory Review Practices Group, 2018). To do so, clinically relevant data that supports the long-term performance and data reproducibility of optical biosensors is necessary. Yet, as aforementioned, these are often overlooked. This data is also crucial in supporting the benefit-risk assessment as part of a marketing authorization application (MAA) to the European Medicines Agency (EMA) (Cerreta et al., 2020). Therefore, a solid evidence base demonstrating the reliability, accuracy, validity and clinical relevance of optical biosensors is necessary to convince not only regulatory authorities, but also clinicians and patients.

Furthermore, while optical biosensors have immense potential to improve therapeutic outcomes and patient safety, important ethical issues surrounding the consolidation of biological data inevitably arise. Concerns over data ownership, confidentiality and privacy are challenging but warranted, given the growing number of cases of data breach and abuse. These devices must abide to regional data protection legislatures in order to assure users that their sensitive medical information will be kept safe. Otherwise, it is unlikely that the device will be widely adopted by clinicians and patients, squandering time and money invested into its research and development.

\section{Looking towards the future}

Technological advancements in the field of materials engineering, biotechnology and nanotechnology continue to contribute significantly towards the vision of routine TDM powered by optical biosensors. Improvements to molecularly imprinted polymers and new aptamer formats, such as xeno nucleic acid-based aptamers, offer solutions to existing stability issues (Eremeeva et al., 2019; Kupai et al., 2017). New optical biosensing configurations continue to emerge with increasing sensitivity and responsiveness. The stability, safety and cost-effectiveness of nanoparticles are benefitting from ongoing research in nanomaterials and nanofabrication technologies, which could facilitate the transition of TDM optical biosensors to clinical settings.

However, as with many emerging fields, current research often involves increasingly complicated formats or fabrication techniques that usually require strictly controlled environmental conditions to perform (Leroux, 2018). These are frequently achievable only in laboratory settings and are unsuitable for translation into the commercial market and clinics. Therefore, while there is certainly a need for technical improvements, the importance of applicability should not be forsaken. Indeed, future research should pay equal attention to critically evaluate their work in clinical samples and should utilize scalable and cost-effective technologies. Effort should also be invested into ensuring that the sensors are easy to use by minimally trained users or even by the patients themselves, thereby lowering the barrier to adoption. Frequent crosstalk between academics, clinicians and regulators to establish a consensus on the clinical need and regulatory requirements would help accelerate the transition of optical biosensors from benches into clinics.

In addition, as we are amid the Fourth Industrial Revolution, there are numerous emerging technologies that can complement optical biosensors to enhance their performance and feasibility. As aforementioned, optical biosensors can be integrated with existing smartphones which can then be connected to a cloud. This enables seamless transmission of relevant information to clinicians without needing face-toface meetings, which significantly improves access to healthcare in remote regions where clinical expertise would not otherwise be available. Indeed, the internet of things (IoT) can facilitate a decentralized healthcare system that improves efficiency, accessibility and quality of healthcare services (Abimbola et al., 2019; Morales-Narváez and Dincer, 2020). Furthermore, machine learning and artificial intelligence have the potential to boost the diagnostic and therapeutic accuracy of optical biosensors by improving pattern analysis and classifications (Jin et al., 2020). Indeed, these technologies have already been demonstrated for evaluating the accuracy of detection, solving spectral overlap, and extracting vibrational data, amongst others (Kühner et al., 2019; Saberi et al., 2020). To secure these sensitive data, blockchain technology could be employed.

\section{Conclusion}

Optical biosensors show great promise in revolutionizing TDM practices and facilitating the transition into a new era of personalized 
medicine. Complemented by the bespoke medicine fabrication capabilities of 3D printing, the archaic one-size-fit-all model will eventually be superseded by precision dosing, and accordingly improved patient outcomes. Optical biosensors afford high sensitivity, efficiency, portability and potential affordability, that make them capable of becoming key clinical and commercial tools for TDM. These drug monitoring biosensors could also see their application expand beyond TDM, such as in clinical trials, illicit drug tests, and emerging responsive drug delivery technologies. In this review, salient techniques used in optical biosensors were summarized with prominent studies that demonstrate the progress towards clinical translation. Admittedly, there remains outstanding technical and regulatory hurdles that impede their adoption, such as robustness, safety, and reliability. Nonetheless, steady advances in materials science and biotechnology holds promise in overcoming these challenges. Integration with IoT and other emerging digital health technologies will also enhance the accessibility and quality of TDM services. With greater collaboration between researchers and regulatory authorities, we anticipate that optical biosensors will unlock the full potential of TDM, enabling patients to receive accurate drug doses tailored to their unique biology.

\section{Declaration of competing interest}

The authors declare that they have no known competing financial interests or personal relationships that could have appeared to influence the work reported in this paper.

\section{Acknowledgements}

The authors thank the Engineering and Physical Sciences Research Council (EPSRC), UK for their financial support (EP/R513143/1 and EP/ S00900/1).

\section{References}

Abimbola, S., Baatiema, L., Bigdeli, M., 2019. The impacts of decentralization on health system equity, efficiency and resilience: a realist synthesis of the evidence. Health Pol. Plann. 34 (8), 605-617.

Abnous, K., Danesh, N.M., Ramezani, M., Emrani, A.S., Taghdisi, S.M., 2016. A novel colorimetric sandwich aptasensor based on an indirect competitive enzyme-free method for ultrasensitive detection of chloramphenicol. Biosens. Bioelectron. 78, $80-86$.

Aboul-Enein, H.Y., Stefan, R.-I., van Staden, J.F., 1999. Chemiluminescence-based (Bio) Sensors - an overview. Crit. Rev. Anal. Chem. 29 (4), 323-331.

Afsharipour, R., Haji Shabani, A.M., Dadfarnia, S., Kazemi, E., 2019. Selective fluorometric determination of sulfadiazine based on the growth of silver nanoparticles on graphene quantum dots. Microchimica Acta 187 (1), 54.

Ahmadpour, H., Hosseini, S.M.M., 2019. A solid-phase luminescence sensor based on molecularly imprinted polymer-CdSeS/ZnS quantum dots for selective extraction and detection of sulfasalazine in biological samples. Talanta 194, 534-541.

Ahmed, S., Zhou, Z., Zhou, J., Chen, S.-Q., 2016. Pharmacogenomics of drug metabolizing enzymes and transporters: relevance to precision medicine. Dev. Reprod. Biol. 14 (5), 298-313.

Ahmed, S.W., Anwar, H., Shama, Siddiqui, A., Shah, M.R., Ahmed, A., Ali, S.A., 2018. Synthesis and chemosensing of nitrofurazone using olive oil based silver nanoparticles (O-AgNPs). Sensor. Actuator. B Chem. 256, 429-439.

Akbari Javar, H., Garkani-Nejad, Z., Dehghannoudeh, G., Mahmoudi-Moghaddam, H., 2020. Development of a new electrochemical DNA biosensor based on Eu3+-doped $\mathrm{NiO}$ for determination of amsacrine as an anti-cancer drug: electrochemical, spectroscopic and docking studies. Anal. Chim. Acta 1133, 48-57.

Al-Hetlani, E., Amin, M.O., Madkour, M., 2018. Novel and versatile solid-state chemiluminescence sensor based on TiO2-Ru(bpy) $32+$ nanoparticles for pharmaceutical drugs detection. Nanophotonics 7 (3), 683-692.

Al-Hetlani, E., D’Cruz, B., Amin, M.O., 2020. A 3D miniaturized solid-state chemiluminescence sensor based on ruthenium functionalized polymeric monolith for the detection of pharmaceutical drugs. J. Mater. Sci. 55 (27), 13232-13243.

Alam, A.-M., Kamruzzaman, M., Lee, S.H., Kim, Y.H., Kim, S.Y., Kim, G.M., Jo, H.J., Kim, S.H., 2012. Determination of catecholamines based on the measurement of the metal nanoparticle-enhanced fluorescence of their terbium complexes. Microchimica Acta 176 (1), 153-161.

Alarfaj, N.A., Aly, F.A., Al-Tamimi, S.A., El-Tohamy, M.F., 2013. Enhanced silver nanoparticle chemiluminescence method for the determination of gemifloxacin mesylate using sequential injection analysis. J. Chem. Soc. Pakistan 35 (5), 13-19.
Alarfaj, N.A., Aly, F.A., El-Tohamy, M.F., 2015. Application of silver nanoparticles to the chemiluminescence determination of cefditoren pivoxil using the luminol-ferricyanide system. Luminescence 30 (1), 91-97.

Ali, M.F.B., Marzouq, M.A., Salman, B.I., Hussein, S.A., 2019. Utility of surface plasmon resonance response of silver nanoparticles for assay of Teicoplanin in human plasma using spectrofluorimetric technique. Microchem. J. 146, 187-191.

Ali, M.F.B., Salman, B.I., Hussein, S.A., Marzouq, M.A., 2020. Applicability of ninhydrin as a fluorescent reagent for estimation of teicoplanin in human plasma using saltingout assisted liquid-liquid extraction technique. Luminescence 35 (7), 1118-1124.

Amjadi, M., Farzampour, L., 2014. Selective turn-on fluorescence assay of 6-thioguanine by using harmine-modified silver nanoparticles. Luminescence 29 (6), 689-694.

Amjadi, M., Jalili, R., 2016. Molecularly imprinted polymer-capped nitrogen-doped graphene quantum dots as a novel chemiluminescence sensor for selective and sensitive determination of doxorubicin. RSC Adv. 6 (89), 86736-86743.

Amjadi, M., Manzoori, J.L., Hassanzadeh, J., Sorouraddin, M.H., 2013. Permanganate-bromide-silver nanoparticles as a new chemiluminescence system and its application to captopril determination. Talanta 115, 600-605.

Anwar, A., Shah, M.R., Muhammad, S.P., Ali, K., Khan, N.A., 2019. Synthesis of 4-formyl pyridinium propylthioacetate stabilized silver nanoparticles and their application in chemosensing of 6-aminopenicillanic acid (APA). Int. J. Environ. Sci. Technol. 16 (3), 1563-1570.

Arunjegan, A., Rajaji, P., Sivanesan, S., Panneerselvam, P., 2021. A Turn-ON fluorometric biosensor based on ssDNA immobilized with a metal phenolic nanomaterial for the sequential detection of $\mathrm{Pb}(\mathrm{ii})$ and epirubicin cancer drug. RSC Adv. 11 (20), 12361-12373.

Ates, H.C., Roberts, J.A., Lipman, J., Cass, A.E.G., Urban, G.A., Dincer, C., 2020. On-site therapeutic drug monitoring. Trends Biotechnol. 38 (11), 1262-1277.

Bai, Y., Su, Q., Xiao, J., Feng, F., Yang, X., 2020. Exploration of Synthesizing Fluorescent Silicon Nanoparticles and Label-free Detection of Sulfadiazine Sodium. Talanta, 121410.

Balaji, A., Zhang, J., 2017. Electrochemical and optical biosensors for early-stage cancer diagnosis by using graphene and graphene oxide. Cancer Nanotechnology 8 (1), 10.

Ball, D.W., 2006. Field Guide to Spectroscopy. Spie Press Bellingham, Washington.

BBC News, 2018. Ugandan Wins Africa Prize for Bloodless Malaria Test.

Beeg, M., Nobili, A., Orsini, B., Rogai, F., Gilardi, D., Fiorino, G., Danese, S., Salmona, M., Garattini, S., Gobbi, M., 2019. A Surface Plasmon Resonance-based assay to measure serum concentrations of therapeutic antibodies and anti-drug antibodies. Sci. Rep. 9 (1), 2064.

Berger, A.G., Restaino, S.M., White, I.M., 2017. Vertical-flow paper SERS system for therapeutic drug monitoring of flucytosine in serum. Anal. Chim. Acta 949, 59-66.

Berthold Technologies, 2020. BRET (Bioluminescence Resonance Energy Transfer) Berthold Technologies. Berthold Technologies GmbH \& Co.KG.

Bhalla, N., Jamshaid, A., Leung, M.H.M., Ishizu, N., Shen, A.Q., 2019. Electrical contact of metals at the nanoscale overcomes the oxidative susceptibility of silver-based nanobiosensors. ACS Applied Nano Materials 2 (4), 2064-2075.

Bian, S., Lu, J., Delport, F., Vermeire, S., Spasic, D., Lammertyn, J., Gils, A., 2018. Development and validation of an optical biosensor for rapid monitoring of adalimumab in serum of patients with Crohn's disease. Drug Test. Anal. 10 (3), 592-596.

Bindesri, S.D., Alhatab, D.S., Brosseau, C.L., 2018. Development of an electrochemical surface-enhanced Raman spectroscopy (EC-SERS) fabric-based plasmonic sensor for point-of-care diagnostics. Analyst 143 (17), 4128-4135.

Bosch, M.E., Sánchez, A.J.R., Rojas, F.S., Ojeda, C.B., 2007. Recent development in optical fiber biosensors. Sensors 7 (6), 797-859.

Buclin, T., Thoma, Y., Widmer, N., André, P., Guidi, M., Csajka, C., Decosterd, L.A., 2020. The steps to therapeutic drug monitoring: a structured approach illustrated with imatinib. Front. Pharmacol. 11 (177).

Capel, A.J., Rimington, R.P., Lewis, M.P., Christie, S.D.R., 2018. 3D printing for chemical, pharmaceutical and biological applications. Nature Reviews Chemistry 2 (12), 422-436.

Carrasco, S., Benito-Peña, E., Walt, D.R., Moreno-Bondi, M.C., 2015. Fiber-optic array using molecularly imprinted microspheres for antibiotic analysis. Chem. Sci. 6 (5), 3139-3147.

Carrasco-Ramiro, F., Peiró-Pastor, R., Aguado, B., 2017. Human genomics projects and precision medicine. Gene Ther. 24 (9), 551-561.

Cerreta, F., Ritzhaupt, A., Metcalfe, T., Askin, S., Duarte, J., Berntgen, M., Vamvakas, S., 2020. Digital technologies for medicines: shaping a framework for success. Nat. Rev. Drug Discov. 19, 573-574.

Charbgoo, F., Soltani, F., Taghdisi, S.M., Abnous, K., Ramezani, M., 2016. Nanoparticles application in high sensitive aptasensor design. Trac. Trends Anal. Chem. 85, 85-97.

Chavada, V.D., Bhatt, N.M., Sanyal, M., Shrivastav, P.S., 2017. Pyrophosphate functionalized silver nanoparticles for colorimetric determination of deferiprone via competitive binding to Fe(III). Microchimica Acta 184 (10), 4203-4208.

Chen, C., Wang, J., 2020. Optical biosensors: an exhaustive and comprehensive review. Analyst 145 (5), 1605-1628.

D’Elia, V., Rubio-Retama, J., Ortega-Ojeda, F.E., García-Ruiz, C., Montalvo, G., 2018. Gold nanorods as SERS substrate for the ultratrace detection of cocaine in nonpretreated oral fluid samples. Colloid. Surface. Physicochem. Eng. Aspect. 557, $43-50$.

Dai, Y., Zhang, Y., Liao, W., Wang, W., Wu, L., 2020. G-quadruplex specific thioflavin Tbased label-free fluorescence aptasensor for rapid detection of tetracycline. Spectrochim. Acta Mol. Biomol. Spectrosc. 238, 118406.

Damborský, P., Švitel, J., Katrlík, J., 2016. Optical biosensors. Essays Biochem. 60 (1), 91-100. 
de-los-Santos-Álvarez, N., Lobo-Castañón, M.J., Miranda-Ordieres, A.J., TuñónBlanco, P., 2009. SPR sensing of small molecules with modified RNA aptamers: detection of neomycin B. Biosens. Bioelectron. 24 (8), 2547-2553.

Deng, B., Luo, X., Zhang, M., Ye, L., Chen, Y., 2019. Quantitative detection of acyclovir by surface enhanced Raman spectroscopy using a portable Raman spectrometer coupled with multivariate data analysis. Colloids Surf. B Biointerfaces 173, 286-294.

Dillon, P.P., Daly, S.J., Manning, B.M., O'Kennedy, R., 2003. Immunoassay for the determination of morphine-3-glucuronide using a surface plasmon resonance-based biosensor. Biosens. Bioelectron. 18 (2), 217-227.

Ding, Y., Zhang, X., Yin, H., Meng, Q., Zhao, Y., Liu, L., Wu, Z., Xu, H., 2017. Quantitative and sensitive detection of chloramphenicol by surface-enhanced Raman scattering. Sensors 17 (12), 2962.

Dong, Z., Huang, G., Xu, S., Deng, C., Zhu, J., Chen, S., Yang, X., Zhao, S., 2009. Realtime and label-free detection of chloramphenicol residues with specific molecular interaction. J. Microsc. 234 (3), 255-261.

Dou, Y., Yang, X., Liu, Z., Zhu, S., 2013. Homocysteine-functionalized silver nanoparticles for selective sensing of $\mathrm{Cu} 2+$ ions and Lidocaine hydrochloride. Colloid. Surface. Physicochem. Eng. Aspect. 423, 20-26.

Duval, D., Lechuga, L.M., 2015. Optical Waveguide Biosensors.

El-Zahry, M.R., Refaat, I.H., Mohamed, H.A., Rosenberg, E., Lendl, B., 2015. Utility of surface enhanced Raman spectroscopy (SERS) for elucidation and simultaneous determination of some penicillins and penicilloic acid using hydroxylamine silver nanoparticles. Talanta 144, 710-716.

Elbadawi, M., Muniz Castro, B., Gavins, F.K.H., Ong, J.J., Gaisford, S., Perez, G., Basit, A. W., Cabalar, P., Goyanes, A., 2020. M3DISEEN: a novel machine learning approach for predicting the 3D printability of medicines. Int. J. Pharm. 590, 119837.

Elbadawi, M., Ong, J.J., Pollard, T.D., Gaisford, S., Basit, A.W., 2021. Additive manufacturable materials for electrochemical biosensor electrodes. Adv. Funct. Mater. 31 (10), 2006407.

Eleftheriadis, G.K., Kantarelis, E., Monou, P.K., Andriotis, E.G., Bouropoulos, N., Tzimtzimis, E.K., Tzetzis, D., Rantanen, J., Fatouros, D.G., 2021. Automated digital design for 3D-printed individualized therapies. Int. J. Pharm. 599, 120437.

Emdadi, S., Sorouraddin, M.H., Denanny, L., 2021. Enhanced chemiluminescence determination of paracetamol. Analyst 146 (4), 1326-1333.

Emmerson, G.D., Gawith, C.B.E., Smith, P.G.R., 2010. Multiwavelength Optical Sensors, Google Patents.

Ensafi, A.A., Nasr-Esfahani, P., Rezaei, B., 2017. Simultaneous detection of folic acid and methotrexate by an optical sensor based on molecularly imprinted polymers on dualcolor CdTe quantum dots. Anal. Chim. Acta 996, 64-73.

Eremeeva, E., Fikatas, A., Margamuljana, L., Abramov, M., Schols, D., Groaz, E., Herdewijn, P., 2019. Highly stable hexitol based XNA aptamers targeting the vascular endothelial growth factor. Nucleic Acids Res. 47 (10), 4927-4939.

Faham, S., Golmohammadi, H., Ghavami, R., Khayatian, G., 2019. A nanocellulose-based colorimetric assay kit for smartphone sensing of iron and iron-chelating deferoxamine drug in biofluids. Anal. Chim. Acta 1087, 104-112.

Farkouh, A., Riedl, T., Gottardi, R., Czejka, M., Kautzky-Willer, A., 2020. Sex-related differences in pharmacokinetics and pharmacodynamics of frequently prescribed drugs: a review of the literature. Adv. Ther. 37 (2), 644-655.

Farquharson, S., Shende, C., Inscore, F.E., Maksymiuk, P., Gift, A., 2005. Analysis of 5 fluorouracil in saliva using surface-enhanced Raman spectroscopy. J. Raman Spectrosc. 36 (3), 208-212.

Fei, J., Wu, L., Zhang, Y., Zong, S., Wang, Z., Cui, Y., 2017. Pharmacokinetics-on-a-Chip using label-free SERS technique for programmable dual-drug analysis. ACS Sens. 2 (6), 773-780.

Fernández, F., Hegnerová, K., Piliarik, M., Sanchez-Baeza, F., Homola, J., Marco, M.P., 2010. A label-free and portable multichannel surface plasmon resonance immunosensor for on site analysis of antibiotics in milk samples. Biosens. Bioelectron. 26 (4), 1231-1238.

Fong, K.E., Yung, L.-Y.L., 2013. Localized surface plasmon resonance: a unique property of plasmonic nanoparticles for nucleic acid detection. Nanoscale 5 (24), 12043-12071.

Fornasaro, S., Bonifacio, A., Marangon, E., Buzzo, M., Toffoli, G., Rindzevicius, T., Schmidt, M.S., Sergo, V., 2018. Label-free quantification of anticancer drug imatinib in human plasma with surface enhanced Raman spectroscopy. Anal. Chem. 90 (21), $12670-12677$.

Fornasaro, S., Marta, S.D., Rabusin, M., Bonifacio, A., Sergo, V., 2016. Toward SERSbased point-of-care approaches for therapeutic drug monitoring: the case of methotrexate. Faraday Discuss 187, 485-499, 0.

Fox, J.L., 2015. Obama catapults patient-empowered precision medicine. Nat. Biotechnol. 33 (4), 325, 325.

Frasconi, M., Tel-Vered, R., Riskin, M., Willner, I., 2010. Surface plasmon resonance analysis of antibiotics using imprinted boronic acid-functionalized Au nanoparticle composites. Anal. Chem. 82 (6), 2512-2519.

Fu, E., Chinowsky, T., Nelson, K., Johnston, K., Edwards, T., Helton, K., Grow, M., Miller, J.W., Yager, P., 2007. SPR imaging-based salivary diagnostics system for the detection of small molecule analytes. Ann. N. Y. Acad. Sci. 1098 (1), 335-344.

Gao, J., Zhang, N., Ji, D., Song, H., Liu, Y., Zhou, L., Sun, Z., Jornet, J.M., Thompson, A. C., Collins, R.L., Song, Y., Jiang, S., Gan, Q., 2018a. Superabsorbing metasurfaces with hybrid $\mathrm{Ag}-\mathrm{Au}$ nanostructures for surface-enhanced Raman spectroscopy sensing of drugs and chemicals. Small Methods 2 (7), 1800045.

Gao, M., Li, L., Lu, S., Liu, Q., He, H., 2018b. Silver nanoparticles for the visual detection of lomefloxacin in the presence of cystine. Spectrochim. Acta Mol. Biomol. Spectrosc. 205, 72-78.

Garzón, V., Pinacho, D.G., Bustos, R.-H., Garzón, G., Bustamante, S., 2019. Optical biosensors for therapeutic drug monitoring. Biosensors 9 (4), 132.
Germer, T.A., Zwinkels, J.C., Tsai, B.K., 2014. Chapter 1 - introduction. In: Germer, T.A., Zwinkels, J.C., Tsai, B.K. (Eds.), Experimental Methods in the Physical Sciences. Academic Press, pp. 1-9.

Gomes, H.I.A.S., Sales, M.G.F., 2015. Development of paper-based color test-strip for drug detection in aquatic environment: application to oxytetracycline. Biosens. Bioelectron. 65, 54-61.

Goyanes, A., Madla, C.M., Umerji, A., Duran Piñeiro, G., Giraldez Montero, J.M., Lamas Diaz, M.J., Gonzalez Barcia, M., Taherali, F., Sánchez-Pintos, P., Couce, M.-L., Gaisford, S., Basit, A.W., 2019. Automated therapy preparation of isoleucine formulations using 3D printing for the treatment of MSUD: first single-centre, prospective, crossover study in patients. Int. J. Pharm. 567, 118497.

Griss, R., Schena, A., Reymond, L., Patiny, L., Werner, D., Tinberg, C.E., Baker, D., Johnsson, K., 2014. Bioluminescent sensor proteins for point-of-care therapeutic drug monitoring. Nat. Chem. Biol. 10 (7), 598-603.

Gukowsky, J.C., Tan, C., Han, Z., He, L., 2018. Cysteamine-modified gold nanoparticles as a colorimetric sensor for the rapid detection of gentamicin. J. Food Sci. 83 (6), $1631-1638$.

Guner, H., Ozgur, E., Kokturk, G., Celik, M., Esen, E., Topal, A.E., Ayas, S., Uludag, Y., Elbuken, C., Dana, A., 2017. A smartphone based surface plasmon resonance imaging (SPRi) platform for on-site biodetection. Sensor. Actuator. B Chem. 239, $571-577$.

Guo, L., Yin, Y., Huang, R., Qiu, B., Lin, Z., Yang, H.-H., Li, J., Chen, G., 2012. Enantioselective analysis of melagatran via an LSPR biosensor integrated with a microfluidic chip. Lab Chip 12 (20), 3901-3906.

Gupta, R., Goddard, N.J., 2020. Leaky Waveguides (LWs) for Chemical and Biological Sensing - A Review and Future Perspective. Sensors and Actuators B, Chemical, 128628.

Gustavo González, A., Ángeles Herrador, M., 2007. A practical guide to analytical method validation, including measurement uncertainty and accuracy profiles. Trac. Trends Anal. Chem. 26 (3), 227-238.

Halawa, M.I., Mostafa, I.M., Wu, G., Li, B.S., 2021. Amplified anodic electrogenerated chemiluminescence of tris(2,2'-bipyridyl)ruthenium(II) for ultrasensitive detection of bambuterol: application to content uniformity testing. J. Electroanal. Chem. 880, 114881.

Halilović, A., Merdan, E., Ž, K., Pokvić, L.G., 2019. Review of Biosensors for Environmental Field Monitoring. 2019 8th Mediterranean Conference on Embedded Computing. MECO), pp. 1-8.

Han, S., Li, X., Wei, B., 2014. Silver nanoparticle enhanced chemiluminescence method for the determination of nitrazepam. Anal. Sci. 30 (4), 495-500.

Han, S., Zhang, C., Sha, X., Li, N., Hasi, W., Zhang, Y., 2020. Effective SERS method for identification of dexmedetomidine hydrochloride in biological samples. Analytical Methods 12 (12), 1662-1669.

Hashemi, F., Rastegarzadeh, S., Pourreza, N., 2018. Response surface methodology optimized dispersive liquid-liquid microextraction coupled with surface plasmon resonance of silver nanoparticles as colorimetric probe for determination of captopril. Sensor. Actuator. B Chem. 256, 251-260.

He, J., Li, G., Hu, Y., 2017. Aptamer-involved fluorescence amplification strategy facilitated by directional enzymatic hydrolysis for bioassays based on a metal organic framework platform: highly selective and sensitive determination of thrombin and oxytetracycline. Microchimica Acta 184 (7), 2365-2373.

Hidi, I.J., Mühlig, A., Jahn, M., Liebold, F., Cialla, D., Weber, K., Popp, J., 2014. LOCSERS: towards point-of-care diagnostic of methotrexate. Analytical Methods 6 (12), 3943-3947.

Hossain, A., Canning, J., Ast, S., Rutledge, P.J., Yen, T.L., Jamalipour, A., 2015. Lab-in-aPhone: smartphone-based portable fluorometer for $\mathrm{pH}$ measurements of environmental water. IEEE Sensor. J. 15 (9), 5095-5102.

Hu, S., Liu, J., Zhang, G., Wang, Z., Xiao, X., Li, D., Peng, J., Lai, W., 2020. Reliable performance of aggregation-induced emission nanoparticle-based lateral flow assay for norfloxacin detection in nine types of animal-derived food. Talanta 219, 121245.

Huang, Q.-D., Lv, C.-H., Yuan, X.-L., He, M., Lai, J.-P., Sun, H., 2021. A novel fluorescent optical fiber sensor for highly selective detection of antibiotic ciprofloxacin based on replaceable molecularly imprinted nanoparticles composite hydrogel detector. Sensor. Actuator. B Chem. 328, 129000.

IMDRF Good Regulatory Review Practices Group, 2018. Essential Principles of Safety and Performance of Medical Devices and IVD Medical Devices.

International Medical Device Regulators Forum, GHTF Archive.

Jabbari, S., Dabirmanesh, B., Arab, S.S., Amanlou, M., Daneshjou, S., Gholami, S., Khajeh, K., 2017. A novel enzyme based SPR-biosensor to detect bromocriptine as an ergoline derivative drug. Sensor. Actuator. B Chem. 240, 519-527.

Jalalian, S.H., Taghdisi, S.M., Danesh, N.M., Bakhtiari, H., Lavaee, P., Ramezani, M., Abnous, K., 2015. Sensitive and fast detection of tetracycline using an aptasensor. Analytical Methods 7 (6), 2523-2528.

Jantarat, T., Chuaychob, S., Thammakhet-Buranachai, C., Thavarungkul, P., Kanatharana, P., Srisintorn, W., Buranachai, C., 2021. A label-free DNA-based fluorescent sensor for cisplatin detection. Sensor. Actuator. B Chem. 326, 128764.

Jelliffe, R., Neely, M., Bayard, D., 2020. Chapter 7 - pharmacokinetic methods for TDM data analysis and optimal individualization of drug dosage regimens. In: Hempel, G. (Ed.), Handbook of Analytical Separations. Elsevier Science B.V, pp. 135-168.

Jin, X., Liu, C., Xu, T., Su, L., Zhang, X., 2020. Artificial intelligence biosensors: challenges and prospects. Biosens. Bioelectron. 165, 112412.

Jones, R.R., Hooper, D.C., Zhang, L., Wolverson, D., Valev, V.K., 2019. Raman techniques: fundamentals and frontiers. Nanoscale Research Letters 14 (1), 231.

Jouyban, A., Rahimpour, E., 2020. Optical Sensors Based on Silver Nanoparticles for Determination of Pharmaceuticals: an Overview of Advances in the Last Decade. Talanta, 121071. 
Jouyban, A., Samadi, A., Khoubnasabjafari, M., 2017a. A new "turn-on" fluorescent sensor based on gold quantum dots and silver nanoparticles for lamotrigine detection in plasma. Talanta 172, 126-132.

Jouyban, A., Samadi, A., Khoubnasabjafari, M., Jouyban-Gharamaleki, V., Ranjbar, F., 2017b. Amidosulfonic acid-capped silver nanoparticles for the spectrophotometric determination of lamotrigine in exhaled breath condensate. Microchimica Acta 184 (8), 2991-2998.

JoVE Science Education Database, 2020. Optical Biosensing. Bioengineering. JoVE, Cambridge, MA.

Jubran, A., 2015. Pulse oximetry. Crit. Care 19 (1), 272, 272.

Kamruzzaman, M., Alam, A.-M., Kim, K.M., Lee, S.H., Kim, Y.H., Kim, S.H., 2012. Silver nanoparticle-enhanced chemiluminescence method for determining naproxen based on europium(III)-Sensitized Ce(IV)-Na2S2O4 reaction. J. Fluoresc. 22 (3), 883-890.

Kamruzzaman, M., Alam, A.-M., Lee, S.H., Suh, Y.S., Kim, Y.H., Kim, G.M., Kim, S.H., 2011. Method for determination of fluoroquinolones based on the plasmonic interaction between their fluorescent terbium complexes and silver nanoparticles. Microchimica Acta 174 (3), 353.

Kazmi, S.A., Qureshi, M.Z., Masson, J.-F., 2020. Drug-based gold nanoparticles overgrowth for enhanced SPR biosensing of doxycycline. Biosensors 10 (11).

Khajavian, Z., Esmaelpourfarkhani, M., Ramezani, M., Alibolandi, M., Abnous, K., Taghdisi, S.M., 2021. A highly sensitive, simple and label-free fluorescent aptasensor for tobramycin sensing based on PicoGreen intercalation into DNA duplex regions of three-way junction origami. Microchem. J. 160, 105657.

Khodaveisi, J., Shabani, A.M.H., Dadfarnia, S., Saberi, D., 2017. A novel sensor for determination of naproxen based on change in localized surface plasmon peak of functionalized gold nanoparticles. Spectrochim. Acta Mol. Biomol. Spectrosc. 179, 11-16.

Koczula, K.M., Gallotta, A., 2016. Lateral flow assays. Essays Biochem. 60 (1), 111-120.

Koh, E.H., Lee, W.-C., Choi, Y.-J., Moon, J.-I., Jang, J., Park, S.-G., Choo, J., Kim, D.-H., Jung, H.S., 2021. A wearable surface-enhanced Raman scattering sensor for labelfree molecular detection. ACS Appl. Mater. Interfaces 13 (2), 3024-3032.

Kong, L., Gan, Y., Liang, T., Zhong, L., Pan, Y., Kirsanov, D., Legin, A., Wan, H., Wang, P., 2020. A novel smartphone-based CD-spectrometer for high sensitive and costeffective colorimetric detection of ascorbic acid. Anal. Chim. Acta 1093, 150-159.

Kühner, L., Semenyshyn, R., Hentschel, M., Neubrech, F., Tarín, C., Giessen, H., 2019. Vibrational sensing using infrared nanoantennas: toward the noninvasive quantitation of physiological levels of glucose and fructose. ACS Sens. 4 (8), 1973-1979.

Kupai, J., Razali, M., Buyuktiryaki, S., Kecili, R., Szekely, G., 2017. Long-term stability and reusability of molecularly imprinted polymers. Polym. Chem. 8 (4), 666-673.

Lan, L., Yao, Y., Ping, J., Ying, Y., 2019. Ultrathin transition-metal dichalcogenide nanosheet-based colorimetric sensor for sensitive and label-free detection of DNA. Sensor. Actuator. B Chem. 290, 565-572.

Leroux, J.-C., 2018. The novelty bubble. J. Contr. Release 278, 140-141.

Lertvachirapaiboon, C., Baba, A., Ekgasit, S., Shinbo, K., Kato, K., Kaneko, F., 2018. Transmission surface plasmon resonance techniques and their potential biosensor applications. Biosens. Bioelectron. 99, 399-415.

Li, A., Song, Z., 2014. Study of silver nanoparticles sensitized fluorescence and secondorder scattering of terbium(III)-Pefloxacin mesylate complex and determination of pefloxacin mesylate. Sci. World J., 742935, 2014.

Li, H., Ye, H.-G., Cheng, R., Guo, J., Liang, Z.-B., Li, G., Li, Q., Wang, C.-F., Chen, S., 2021a. Red dual-emissive carbon dots for ratiometric sensing of veterinary drugs. J. Lumin. 118092.

Li, N., Gu, Y., Gao, M., Wang, Z., Xiao, D., Li, Y., Wang, J., He, H., 2014. Label-free silver nanoparticles for visual colorimetric detection of etimicin. Analytical Methods 6 (19), 7906-7911.

Li, R., Wang, W., El-Sayed, E.-S.M., Su, K., He, P., Yuan, D., 2021b. Ratiometric fluorescence detection of tetracycline antibiotic based on a polynuclear lanthanide metal-organic framework. Sensor. Actuator. B Chem. 330, 129314.

Liang, J., Zhang, Z., Zhao, H., Wan, S., Zhai, X., Zhou, J., Liang, R., Deng, Q., Wu, Y., Lin, G., 2018. Simple and rapid monitoring of doxorubicin using streptavidinmodified microparticle-based time-resolved fluorescence immunoassay. RSC Adv. 8 (28), 15621-15631.

Liao, Z., Zhang, Y., Li, Y., Miao, Y., Gao, S., Lin, F., Deng, Y., Geng, L., 2019. Microfluidic chip coupled with optical biosensors for simultaneous detection of multiple analytes: a review. Biosens. Bioelectron. 126, 697-706.

Litti, L., Amendola, V., Toffoli, G., Meneghetti, M., 2016. Detection of low-quantity anticancer drugs by surface-enhanced Raman scattering. Anal. Bioanal. Chem. 408 (8), 2123-2131.

Litti, L., Ramundo, A., Biscaglia, F., Toffoli, G., Gobbo, M., Meneghetti, M., 2019. A surface enhanced Raman scattering based colloid nanosensor for developing therapeutic drug monitoring. J. Colloid Interface Sci. 533, 621-626.

Liu, J., Liu, W., Huang, Y., Zhao, X., Feng, Z., Wang, D., Gong, Z., Fan, M., 2021a. Selfsupporting liquid film as reproducible SERS platform for therapeutic drug monitoring of berberine hydrochloride in human urine. Microchem. J. 165, 106122.

Liu, J., Zhang, J., Wang, M., Su, X., 2021b. Silicon quantum dots based dual-mode fluorometric and colorimetric sensing of D-penicillamine. Talanta 224, 121886.

Liu, X., Yao, Y., Ying, Y., Ping, J., 2019. Recent advances in nanomaterial-enabled screenprinted electrochemical sensors for heavy metal detection. Trac. Trends Anal. Chem. $115,187-202$.

Losoya-Leal, A., Estevez, M.C., Martínez-Chapa, S.O., Lechuga, L.M., 2015. Design of a surface plasmon resonance immunoassay for therapeutic drug monitoring of amikacin. Talanta 141, 253-258.

Lotfi, A., Manzoori, J.L., 2016. Determination of fluoxetine in pharmaceutical and biological samples based on the silver nanoparticle enhanced fluorescence of fluoxetine-terbium complex. Luminescence 31 (7), 1349-1357.
Lotfi, A., Manzoori, J.L., Mohagheghi, A., 2017. Determination of sertraline in pharmaceutical and biological samples using 1, 10-phenanthroline-terbium probe and silver nanoparticles enhanced fluorescence. J. Lumin. 185, 132-140.

Lu, J., Spasic, D., Delport, F., Van Stappen, T., Detrez, I., Daems, D., Vermeire, S., Gils, A., Lammertyn, J., 2017. Immunoassay for detection of infliximab in whole blood using a fiber-optic surface plasmon resonance biosensor. Anal. Chem. 89 (6), 3664-3671.

Luo, Q., Yu, N., Shi, C., Wang, X., Wu, J., 2016. Surface plasmon resonance sensor for antibiotics detection based on photo-initiated polymerization molecularly imprinted array. Talanta 161, 797-803.

Madla, C.M., Gavins, F.K.H., Merchant, H., Orlu, M., Murdan, S., Basit, A.W., 2021. Let's talk about sex: differences in drug therapy in males and females. Adv. Drug Deliv. Rev. https://doi.org/10.1016/j.addr.2021.05.014.

Markina, N.E., Markin, A.V., 2019. Application of aluminum hydroxide for improvement of label-free SERS detection of some cephalosporin antibiotics in urine. Biosensors 9 (3), 91.

Markina, N.E., Volkova, E.K., Zakharevich, A.M., Goryacheva, I.Y., Markin, A.V., 2018. SERS detection of ceftriaxone and sulfadimethoxine using copper nanoparticles temporally protected by porous calcium carbonate. Microchimica Acta 185 (10), 481.

Matsuura, H., Ujiie, K., Duyen, T.T.M., Izutsu, K., Fujiyama, K., Maeda, S., Harada, K., Hirata, K., 2019. Development of a paper-based luminescence bioassay for therapeutic monitoring of aminoglycosides: a proof-of-concept study. Appl. Biochem. Biotechnol. 189 (3), 798-809.

McCoubrey, L.E., Elbadawi, M., Orlu, M., Gaisford, S., Basit, A.W., 2021. Harnessing machine learning for development of microbiome therapeutics. Gut Microb. 13 (1), 1-20. https://doi.org/10.1080/19490976.2021.1872323.

Melocchi, A., Briatico-Vangosa, F., Uboldi, M., Parietti, F., Turchi, M., von Zeppelin, D., Maroni, A., Zema, L., Gazzaniga, A., Zidan, A., 2021. Quality considerations on the pharmaceutical applications of fused deposition modeling 3D printing. Int. J. Pharm. 592, 119901.

Mendosa, D., 2000. Meter memories. How tom, dick, and charlie did it. Diabetes Wellness Letter 1 (6), 1-10.

Meng, J., Tang, X., Zhou, B., Xie, Q., Yang, L., 2017. Designing of ordered twodimensional gold nanoparticles film for cocaine detection in human urine using surface-enhanced Raman spectroscopy. Talanta 164, 693-699.

Mohamadian, E., Shayanfar, A., Khoubnasabjafari, M., Jouyban-Gharamaleki, V., Ghaffary, S., Jouyban, A., 2017. Analysis of deferiprone in exhaled breath condensate using silver nanoparticle-enhanced terbium fluorescence. Analytical Methods 9 (38), 5640-5645.

Morales-Narváez, E., Dincer, C., 2020. The impact of biosensing in a pandemic outbreak: COVID-19. Biosens. Bioelectron. 163, 112274.

Mu, F., Zhou, X., Fan, F., Chen, Z., Shi, G., 2021. A fluorescence biosensor for therapeutic drug monitoring of vancomycin using in vivo microdialysis. Anal. Chim. Acta 1151, 338250.

Muneer, S., Ayoko, G.A., Islam, N., Izake, E.L., 2020a. Utilizing the thiol chemistry of biomolecules for the rapid determination of anti-TNF- $\alpha$ drug in blood. Talanta 208, 120411.

Muneer, S., Sarfo, D.K., Ayoko, G.A., Islam, N., Izake, E.L., 2020b. Gold-deposited nickel foam as recyclable plasmonic sensor for therapeutic drug monitoring in blood by surface-enhanced Raman spectroscopy. Nanomaterials 10 (9), 1756.

Nag, P., Sadani, K., Mukherji, S., Mukherji, S., 2020. Beta-lactam antibiotics induced bacteriolysis on LSPR sensors for assessment of antimicrobial resistance and quantification of antibiotics. Sensor. Actuator. B Chem. 311, 127945.

Nagel, B., Dellweg, H., Gierasch, L.M., 1992. Glossary for chemists of terms used in biotechnology (IUPAC Recommendations 1992). Pure Appl. Chem. 64 (1), 143-168.

Nguyen, D.K., Jang, C.-H., 2021. A label-free liquid crystal biosensor based on specific DNA aptamer probes for sensitive detection of amoxicillin antibiotic. Micromachines $12(4)$.

Nie, F., Lu, J., He, Y., Du, J., 2005. Use of molecule imprinting-chemiluminescence method for the determination of tamoxifen in breast cancer sufferers' urine. Luminescence 20 (4-5), 315-320.

Nikfarjam, A., Rezayan, A.H., Mohammadkhani, G., Mohammadnejad, J., 2017. Labelfree detection of digoxin using localized surface plasmon resonance-based nanobiosensor. Plasmonics 12 (1), 157-164.

Omar, M.A., Nagy, D.M., Hammad, M.A., Aly, A.A., 2013. Highly sensitive spectrofluorimetric method for determination of certain aminoglycosides in pharmaceutical formulations and human plasma. AAPS PharmSciTech 14 (2), 828-837.

Orooji, Y., Haddad Irani-nezhad, M., Hassandoost, R., Khataee, A., Rahim Pouran, S., Joo, S.W., 2020. Cerium doped magnetite nanoparticles for highly sensitive detection of metronidazole via chemiluminescence assay. Spectrochim. Acta Mol. Biomol. Spectrosc. 234, 118272.

Panikar, S.S., Banu, N., Escobar, E.-R., García, G.-R., Cervantes-Martínez, J., Villegas, T.C., Salas, P., De la Rosa, E., 2020. Stealth Modified Bottom up SERS Substrates for Label-free Therapeutic Drug Monitoring of Doxorubicin in Blood Serum. Talanta, 121138.

Panikar, S.S., Ramírez-García, G., Sidhik, S., Lopez-Luke, T., Rodriguez-Gonzalez, C., Ciapara, I.H., Castillo, P.S., Camacho-Villegas, T., De la Rosa, E., 2019. Ultrasensitive SERS substrate for label-free therapeutic-drug monitoring of paclitaxel and cyclophosphamide in blood serum. Anal. Chem. 91 (3), 2100-2111.

Peláez, E.C., Estevez, M.C., Portela, A., Salvador, J.P., Marco, M.P., Lechuga, L.M., 2018. Nanoplasmonic biosensor device for the monitoring of acenocoumarol therapeutic drug in plasma. Biosens. Bioelectron. 119, 149-155.

Peláez-Gutierrez, E.C., Estévez, M.C., Salvador, J.P., Marco, M.P., Lechuga, L.M., 2017. Localised Surface Plasmon Resonance Biosensor for the Monitoring of Sintrom( Therapeutic Drug in Plasma. 
Pollard, T.D., Ong, J.J., Goyanes, A., Orlu, M., Gaisford, S., Elbadawi, M., Basit, A.W., 2021. Electrochemical biosensors: a nexus for precision medicine. Drug Discov. Today 26 (1), 69-79.

Puscasu, A., Zanchetta, M., Posocco, B., Bunka, D., Tartaggia, S., Toffoli, G., 2021. Development and validation of a selective SPR aptasensor for the detection of anticancer drug irinotecan in human plasma samples. Anal. Bioanal. Chem. 413 (4), 1225-1236.

Qin, G., Wang, J., Li, L., Yuan, F., QingqingZha, Bai, W., Ni, Y., 2020. Highly WaterStable Cd-MOF/Tb3+ Ultrathin Fluorescence Nanosheets for Ultrasensitive and Selective Detection of Cefixime. Talanta, p. 121421.

Qu, J.-c., Chang, Y.-p., Ma, Y.-h., Zheng, J.-m., Li, H.-h., Ou, Q.-q., Ren, C., Chen, X.-g., 2012. A simple and sensitive colorimetric method for the determination of propafenone by silver nanoprobe. Sensor. Actuator. B Chem. 174, 133-139.

Raj, P., Lee, S.-y., Lee, T.Y., 2021. Carbon dot/naphthalimide based ratiometric fluorescence biosensor for hyaluronidase detection. Materials 14 (5).

Raksawong, P., Chullasat, K., Nurerk, P., Kanatharana, P., Davis, F., Bunkoed, O., 2017. A hybrid molecularly imprinted polymer coated quantum dot nanocomposite optosensor for highly sensitive and selective determination of salbutamol in animal feeds and meat samples. Anal. Bioanal. Chem. 409 (20), 4697-4707.

Ramezani, M., Mohammad Danesh, N., Lavaee, P., Abnous, K., Mohammad Taghdisi, S. 2015. A novel colorimetric triple-helix molecular switch aptasensor for ultrasensitive detection of tetracycline. Biosens. Bioelectron. 70, 181-187.

Ramos, I.I., Carl, P., Schneider, R.J., Segundo, M.A., 2019. Automated lab-on-valve sequential injection ELISA for determination of carbamazepine. Anal. Chim. Acta 1076, 91-99.

Rao, H., Liu, W., He, K., Zhao, S., Lu, Z., Zhang, S., Sun, M., Zou, P., Wang, X., Zhao, Q. Wang, Y., Liu, T., 2020. Smartphone-based fluorescence detection of Al3+ and $\mathrm{H} 2 \mathrm{O}$ based on the use of dual-emission biomass carbon dots. ACS Sustain. Chem. Eng. 8 (23), 8857-8867.

Ray, J.V., Mirata, F., Pérollier, C., Arotcarena, M., Bayoudh, S., Resmini, M., 2016. Smart coumarin-tagged imprinted polymers for the rapid detection of tamoxifen. Anal. Bioanal. Chem. 408 (7), 1855-1861.

Ren, X., Li, X., 2020. Flower-like Ag coated with molecularly imprinted polymers as a surface-enhanced Raman scattering substrate for the sensitive and selective detection of glibenclamide. Analytical Methods 12 (22), 2858-2864.

Roda, A., Arduini, F., Mirasoli, M., Zangheri, M., Fabiani, L., Colozza, N., Marchegiani, E., Simoni, P., Moscone, D., 2020. A challenge in biosensors: is it better to measure a photon or an electron for ultrasensitive detection? Biosens. Bioelectron. 155, 112093.

Rodensky, P.L., Wasserman, F., 1964. Effect of sex difference in digoxin toxicity. Am. Heart J. 67 (6), 845.

Ruppert, C., Phogat, N., Laufer, S., Kohl, M., Deigner, H.-P., 2019. A smartphone readout system for gold nanoparticle-based lateral flow assays: application to monitoring of digoxigenin. Microchimica Acta 186 (2), 119.

Saberi, Z., Rezaei, B., Rezaei, P., Ensafi, A.A., 2020. Design a fluorometric aptasensor based on $\mathrm{CoOOH}$ nanosheets and carbon dots for simultaneous detection of lysozyme and adenosine triphosphate. Spectrochim. Acta Mol. Biomol. Spectrosc. 233, 118197.

Saleh, T.A., Al-Shalalfeh, M.M., Al-Saadi, A.A., 2018. Silver loaded graphene as a substrate for sensing 2-thiouracil using surface-enhanced Raman scattering. Sensor. Actuator. B Chem. 254, 1110-1117.

Sarreshtehdar Emrani, A., Danesh, N.M., Lavaee, P., Jalalian, S.H., Ramezani, M., Abnous, K., Taghdisi, S.M., 2015. Sensitive and selective detection of digoxin based on fluorescence quenching and colorimetric aptasensors. Analytical Methods 7 (8), 3419-3424.

Scognamiglio, V., Arduini, F., Palleschi, G., Rea, G., 2014. Biosensing technology for sustainable food safety. Trac. Trends Anal. Chem. 62, 1-10.

Seoane-Viaño, I., Januskaite, P., Alvarez-Lorenzo, C., Basit, A.W., Goyanes, A., 2021a. Semi-solid extrusion 3D printing in drug delivery and biomedicine: personalised solutions for healthcare challenges. J. Contr. Release 332, 367-389.

Seoane-Viaño, I., Trenfield, S.J., Basit, A.W., Goyanes, A., 2021b. Translating 3D printed pharmaceuticals: from hype to real-world clinical applications. Adv. Drug Deliv. Rev. https://doi.org/10.1016/j.addr.2021.05.003. PMID: 33965461.

Sheikh, S.H., Mulchandani, A., 2001. Continuous-flow fluoro-immunosensor for paclitaxel measurement. Biosens. Bioelectron. 16 (9), 647-652.

Simmons, M.D., Miller, L.M., Sundström, M.O., Johnson, S., 2020. Aptamer-based detection of ampicillin in urine samples. Antibiotics 9 (10).

Song, Z., Yu, L., Sun, Y., He, H., 2019. Visual and spectrophotometric detection of metformin based on the host-guest molecular recognition of cucurbit[6]urilmodified silver nanoparticles. Anal. Bioanal. Chem. 411 (27), 7293-7301.

Stillhart, C., Vučićević, K., Augustijns, P., Basit, A.W., Batchelor, H., Flanagan, T.R., Gesquiere, I., Greupink, R., Keszthelyi, D., Koskinen, M., Madla, C.M., Matthys, C. Miljuš, G., Mooij, M.G., Parrott, N., Ungell, A.-L., de Wildt, S.N., Orlu, M., Klein, S., Müllertz, A., 2020. Impact of gastrointestinal physiology on drug absorption in special populations-An UNGAP review. Eur. J. Pharmaceut. Sci. 147, 105280.

Subaihi, A., Muhamadali, H., Mutter, S.T., Blanch, E., Ellis, D.I., Goodacre, R., 2017a. Quantitative detection of codeine in human plasma using surface-enhanced Raman scattering via adaptation of the isotopic labelling principle. Analyst 142 (7), 1099-1105.

Subaihi, A., Trivedi, D.K., Hollywood, K.A., Bluett, J., Xu, Y., Muhamadali, H., Ellis, D.I., Goodacre, R., 2017b. Quantitative online liquid chromatography-surface-enhanced Raman scattering (LC-SERS) of methotrexate and its major metabolites. Anal. Chem. 89 (12), 6702-6709.

Sun, F., Hung, H.-C., Sinclair, A., Zhang, P., Bai, T., Galvan, D.D., Jain, P., Li, B., Jiang, S., Yu, Q., 2016. Hierarchical zwitterionic modification of a SERS substrate enables realtime drug monitoring in blood plasma. Nat. Commun. 7 (1), 13437.
Tackman, E.C., Trujillo, M.J., Lockwood, T.-L.E., Merga, G., Lieberman, M., Camden, J. P., 2018. Identification of substandard and falsified antimalarial pharmaceuticals chloroquine, doxycycline, and primaquine using surface-enhanced Raman scattering. Analytical Methods 10 (38), 4718-4722.

Tang, Y., Zeng, X., Liang, J., 2010. Surface plasmon resonance: an introduction to a surface spectroscopy technique. J Chem. Educ. 87 (7), 742-746.

Tartaggia, S., Alvau, M.D., Meneghello, A., Casetta, B., Polo, F., Toffoli, G., 2018. Practical fluorimetric assay for the detection of anticancer drug SN-38 in human plasma. J. Pharmaceut. Biomed. Anal. 159, 73-81.

Tartaggia, S., Meneghello, A., Bellotto, O., Poetto, A.S., Zanchetta, M., Posocco, B., Bunka, D., Polo, F., Toffoli, G., 2021. An SPR investigation into the therapeutic drug monitoring of the anticancer drug imatinib with selective aptamers operating in human plasma. Analyst 146 (5), 1714-1724.

Tashkhourian, J., Afsharinejad, M., 2017. A novel colorimetric sensor for sensitive determination of R-citalopram based on the plasmonic properties of silver nanoparticles. New J. Chem. 41 (22), 13881-13888.

Tenaglia, E., Ferretti, A., Decosterd, L.A., Werner, D., Mercier, T., Widmer, N., Buclin, T., Guiducci, C., 2018. Comparison against current standards of a DNA aptamer for the label-free quantification of tobramycin in human sera employed for therapeutic drug monitoring. J. Pharmaceut. Biomed. Anal. 159, 341-347.

The Global Harmonization Task Force, 2012. Principles of Medical Devices Classification.

Udugama, B., Kadhiresan, P., Kozlowski, H.N., Malekjahani, A., Osborne, M., Li, V.Y.C., Chen, H., Mubareka, S., Gubbay, J.B., Chan, W.C.W., 2020. Diagnosing COVID-19: the disease and tools for detection. ACS Nano 14 (4), 3822-3835.

Uzun, L., Turner, A.P.F., 2016. Molecularly-imprinted polymer sensors: realising their potential. Biosens. Bioelectron. 76, 131-144.

van Rosmalen, M., Ni, Y., Vervoort, D.F.M., Arts, R., Ludwig, S.K.J., Merkx, M., 2018. Dual-color bioluminescent sensor proteins for therapeutic drug monitoring of antitumor antibodies. Anal. Chem. 90 (5), 3592-3599.

Vicario, A., Sergo, V., Toffoli, G., Bonifacio, A., 2015. Surface-enhanced Raman spectroscopy of the anti-cancer drug irinotecan in presence of human serum albumin. Colloids Surf. B Biointerfaces 127, 41-46.

Vinarov, Z., Abdallah, M., Agundez, J.A.G., Allegaert, K., Basit, A.W., Braeckmans, M., Ceulemans, J., Corsetti, M., Griffin, B.T., Grimm, M., Keszthelyi, D., Koziolek, M., Madla, C.M., Matthys, C., McCoubrey, L.E., Mitra, A., Reppas, C., Stappaerts, J., Steenackers, N., Trevaskis, N.L., Vanuytsel, T., Vertzoni, M., Weitschies, W., Wilson, C., Augustijns, P., 2021. Impact of gastrointestinal tract variability on oral drug absorption and pharmacokinetics: an UNGAP review. Eur. J. Pharmaceut. Sci. $162,105812$.

Wabaidur, S.m., Alam, S.M., Alothman, Z.A., Mohsin, K., 2015a. Silver nanoparticles enhanced flow injection chemiluminescence determination of gatifloxacin in pharmaceutical formulation and spiked urine sample. Spectrochim. Acta Mol. Biomol. Spectrosc. 144, 170-175.

Wabaidur, S.M., Alam, S.M., Alothman, Z.A., Siddiqui, M.R., Naushad, M., Alqadami, A. A., 2015b. Flow-injection chemiluminescence method for the determination of moxifloxacin in pharmaceutical tablets and human urine using silver nanoparticles sensitized calcein-KMnO4 system. Bioproc. Biosyst. Eng. 38 (9), 1803-1810.

Wang, C., Zeng, L., Li, Z., Li, D., 2017a. Review of optical fibre probes for enhanced Raman sensing. J. Raman Spectrosc. 48 (8), 1040-1055.

Wang, J., Cheng, R., Wang, Y., Sun, L., Chen, L., Dai, X., Pan, J., Pan, G., Yan, Y., 2018. Surface-imprinted fluorescence microspheres as ultrasensitive sensor for rapid and effective detection of tetracycline in real biological samples. Sensor. Actuator. B Chem. 263, 533-542.

Wang, J., Lin, W., Cao, E., Xu, X., Liang, W., Zhang, X., 2017b. Surface plasmon resonance sensors on Raman and fluorescence spectroscopy. Sensors 17 (12), 2719.

Wang, W., Lu, Y.-C., Huang, H., Wang, A.-J., Chen, J.-R., Feng, J.-J., 2015. Facile synthesis of $\mathrm{N}$, S-codoped fluorescent carbon nanodots for fluorescent resonance energy transfer recognition of methotrexate with high sensitivity and selectivity. Biosens. Bioelectron. 64, 517-522.

Wang, X., Li, J., Jian, D., Zhang, Y., Shan, Y., Wang, S., Liu, F., 2021. Paper-based antibiotic sensor (PAS) relying on colorimetric indirect competitive enzyme-linked immunosorbent assay for quantitative tetracycline and chloramphenicol detection. Sensor. Actuator. B Chem. 329, 129173.

Wiedman, G.R., Zhao, Y., Perlin, D.S., 2018. A novel, rapid, and low-volume Assay for therapeutic drug monitoring of posaconazole and other long-chain azole-class Antifungal drugs. mSphere 3 (6), e00623, 00618.

Wilson, D.J., Kumar, A.A., Mace, C.R., 2019. Overreliance on cost reduction as a featured element of sensor design. ACS Sens. 4 (5), 1120-1125.

Wolf, S., Frosch, T., Popp, J., Pletz, M.W., Frosch, T., 2019. Highly sensitive detection of the antibiotic ciprofloxacin by means of fiber enhanced Raman spectroscopy. Molecules 24 (24).

Wu, H.-Y., Cunningham, B.T., 2014. Point-of-care detection and real-time monitoring of intravenously delivered drugs via tubing with an integrated SERS sensor. Nanoscale 6 (10), 5162-5171.

Wu, X.-M., Zhang, J.-H., Feng, Z.-S., Chen, W.-X., Zhang, F., Li, Y., 2020. An ultrasensitive "turn-off" fluorescent sensor for the trace detection of rifampicin based on glutathione-stabilized copper nanoclusters. Analyst 145 (4), 1227-1235.

Xing, Y., Fuss, H., Lademann, J., Huang, M.D., Becker-Ross, H., Florek, S., Patzelt, A., Meinke, M.C., Jung, S., Esser, N., 2018. A new concept of efficient therapeutic drug monitoring using the high-resolution continuum source absorption spectrometry and the surface enhanced Raman spectroscopy. Spectrochim. Acta B Atom Spectrosc. $142,91-96$.

Xu, C., Ying, Y., Ping, J., 2019. Colorimetric aggregation assay for kanamycin using gold nanoparticles modified with hairpin DNA probes and hybridization chain reactionassisted amplification. Microchimica Acta 186 (7), 448. 
Xu, J., Jiao, T., Arumugam, S.S., He, P., Zhu, J., Li, H., Chen, Q., 2020. Quantitative analysis of docetaxel by surface-enhanced Raman spectroscopy (SERS) combined with chemometric models and Ag@ZnO nanoparticles substrates. Microchem.J. 155, 104373.

Xu, Z., Deng, P., Li, J., Xu, L., Tang, S., 2017. Molecularly imprinted fluorescent probe based on FRET for selective and sensitive detection of doxorubicin. Mater. Sci. Eng., B 218, 31-39.

Xue, L., Yu, Q., Griss, R., Schena, A., Johnsson, K., 2017. Bioluminescent antibodies for point-of-care diagnostics. Angew. Chem. Int. Ed. 56 (25), 7112-7116.

Yan, D., Frosch, T., Kobelke, J., Bierlich, J., Popp, J., Pletz, M.W., Frosch, T., 2018. Fiberenhanced Raman sensing of cefuroxime in human urine. Anal. Chem. 90 (22), $13243-13248$.

Yan, X., Li, P., Zhou, B., Tang, X., Li, X., Weng, S., Yang, L., Liu, J., 2017. Optimal hotspots of dynamic surfaced-enhanced Raman spectroscopy for drugs quantitative detection. Anal. Chem. 89 (9), 4875-4881.

Yang, L., Chen, Y., Li, H., Luo, L., Zhao, Y., Zhang, H., Tian, Y., 2015. Application of silver nanoparticles decorated with $\beta$-cyclodextrin in determination of 6-mercaptopurine by surface-enhanced Raman spectroscopy. Analytical Methods 7 (16), 6520-6527.

Yola, M.L., Atar, N., Eren, T., 2014. Determination of amikacin in human plasma by molecular imprinted SPR nanosensor. Sensor. Actuator. B Chem. 198, 70-76.

Yoo, S.M., Lee, S.Y., 2016. Optical biosensors for the detection of pathogenic microorganisms. Trends Biotechnol. 34 (1), 7-25.

Yu, B., Cao, C., Li, P., Mao, M., Xie, Q., Yang, L., 2018. Sensitive and simple determination of zwitterionic morphine in human urine based on liquid-liquid micro-extraction coupled with surface-enhanced Raman spectroscopy. Talanta 186, 427-432.

Yue, S., Sun, X.-T., Wang, Y., Zhang, W.-S., Xu, Z.-R., 2018. Microparticles with size/ charge selectivity and $\mathrm{pH}$ response for SERS monitoring of 6-thioguanine in blood serum. Sensor. Actuator. B Chem. 273, 1539-1547.

Yuen, C., Zheng, W., Huang, Z., 2010. Low-level detection of anti-cancer drug in blood plasma using microwave-treated gold-polystyrene beads as surface-enhanced Raman scattering substrates. Biosens. Bioelectron. 26 (2), 580-584.

Zahra, Q.u.A., Luo, Z., Ali, R., Khan, M.I., Li, F., Qiu, B., 2021. Advances in gold nanoparticles-based colorimetric aptasensors for the detection of antibiotics: an overview of the past decade. Nanomaterials 11 (4), 840.
Zaki, N.F.W., Yousif, M., BaHammam, A.S., Spence, D.W., Bharti, V.K., Subramanian, P., Pandi-Perumal, S.R., 2019. Chronotherapeutics: recognizing the importance of timing factors in the treatment of disease and sleep disorders. Clin. Neuropharmacol. 42 (3), 80-87.

Zengin, A., Tamer, U., Caykara, T., 2014. Extremely sensitive sandwich assay of kanamycin using surface-enhanced Raman scattering of 2-mercaptobenzothiazole labeled gold@silver nanoparticles. Anal. Chim. Acta 817, 33-41.

Zeni, L., Perri, C., Cennamo, N., Arcadio, F., D’Agostino, G., Salmona, M., Beeg, M., Gobbi, M., 2020. A portable optical-fibre-based surface plasmon resonance biosensor for the detection of therapeutic antibodies in human serum. Sci. Rep. 10 (1), 11154.

Zhang, W.-S., Wang, Y.-N., Wang, Y., Xu, Z.-R., 2019. Highly reproducible and fast detection of 6-thioguanine in human serum using a droplet-based microfluidic SERS system. Sensor. Actuator. B Chem. 283, 532-537.

Zhang, Y., Lv, M., Gao, P., Zhang, G., Shi, L., Yuan, M., Shuang, S., 2021. The synthesis of high bright silver nanoclusters with aggregation-induced emission for detection of tetracycline. Sensor. Actuator. B Chem. 326, 129009.

Zhao, H., Hasi, W., Bao, L., Han, S., Sha, X., Sun, J., Lou, X., Lin, D., Lv, Z., 2017. Rapid detection of sildenafil drugs in liquid nutraceuticals based on surface-enhanced Raman spectroscopy technology. Chin. J. Chem. 35 (10), 1522-1528.

Zhao, S.S., Bichelberger, M.A., Colin, D.Y., Robitaille, R., Pelletier, J.N., Masson, J.-F., 2012. Monitoring methotrexate in clinical samples from cancer patients during chemotherapy with a LSPR-based competitive sensor. Analyst 137 (20), 4742-4750.

Zhao, S.S., Bukar, N., Toulouse, J.L., Pelechacz, D., Robitaille, R., Pelletier, J.N., Masson, J.-F., 2015. Miniature multi-channel SPR instrument for methotrexate monitoring in clinical samples. Biosens. Bioelectron. 64, 664-670.

Zhu, Q., Yu, X., Wu, Z., Lu, F., Yuan, Y., 2018. Antipsychotic drug poisoning monitoring of clozapine in urine by using coffee ring effect based surface-enhanced Raman spectroscopy. Anal. Chim. Acta 1014, 64-70.

Zhu, Y., Qu, C., Kuang, H., Xu, L., Liu, L., Hua, Y., Wang, L., Xu, C., 2011. Simple, rapid and sensitive detection of antibiotics based on the side-by-side assembly of gold nanorod probes. Biosens. Bioelectron. 26 (11), 4387-4392.

Zimmermann, M., Zimmermann-Kogadeeva, M., Wegmann, R., Goodman, A.L., 2019. Mapping human microbiome drug metabolism by gut bacteria and their genes. Nature 570 (7762), 462-467.

Zourob, M., Goddard, N.J., 2005. Metal clad leaky waveguides for chemical and biosensing applications. Biosens. Bioelectron. 20 (9), 1718-1727. 